Geometry $\&$ Topology

Volume 9 (2005) 1381-1441

Published: 5 August 2005

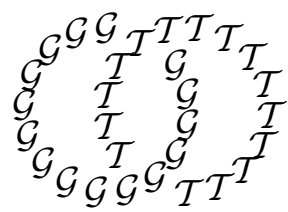

\title{
Automorphisms and abstract commensurators of 2-dimensional Artin groups
}

\author{
JOHN CRISP \\ IMB(UMR 5584 du CNRS), Université de Bourgogne \\ BP 47 870, 21078 Dijon, France \\ Email: jcrisp@u-bourgogne.fr \\ URL: http://math.u-bourgogne.fr/IMB/crisp
}

\begin{abstract}
In this paper we consider the class of 2-dimensional Artin groups with connected, large type, triangle-free defining graphs (type CLTTF). We classify these groups up to isomorphism, and describe a generating set for the automorphism group of each such Artin group. In the case where the defining graph has no separating edge or vertex we show that the Artin group is not abstractly commensurable to any other CLTTF Artin group. If, moreover, the defining graph satisfies a further "vertex rigidity" condition, then the abstract commensurator group of the Artin group is isomorphic to its automorphism group and generated by inner automorphisms, graph automorphisms (induced from automorphisms of the defining graph), and the involution which maps each standard generator to its inverse.

We observe that the techniques used here to study automorphisms carry over easily to the Coxeter group situation. We thus obtain a classification of the CLTTF type Coxeter groups up to isomorphism and a description of their automorphism groups analogous to that given for the Artin groups.
\end{abstract}

AMS Classification numbers Primary: 20F36, 20F55

Secondary: 20F65, 20F67

Keywords: 2-dimensional Artin group, Coxeter group, commensurator group, graph automorphisms, triangle free

Proposed: Joan Birman

Received: 18 December 2004

Seconded: Walter Neumann, Martin Bridson

Revised: 2 August 2005

(c) Geometry $8 \mathcal{G}$ Topology Publications 


\section{Introduction and statement of results}

Let $\Delta$ denote a simplicial graph with vertex set $V(\Delta)$ and edge set $E(\Delta) \subset$ $V(\Delta) \times V(\Delta)$. Suppose also that every edge $e=\{s, t\} \in E(\Delta)$ carries a label $m_{e}=m_{s t} \in \mathbb{N}_{\geq 2}$. We define the Artin group $G(\Delta)$ associated to the (labelled) defining graph $\Delta$ to be the group given by the presentation 1

$$
G(\Delta)=\langle V(\Delta)| \underbrace{s t s t s \cdots}_{m_{s t}}=\underbrace{t s t s t \cdots}_{m_{s t}} \text { for all }\{s, t\} \in E(\Delta)\rangle .
$$

Adding the relations $s^{2}=1$ for each $s \in V(\Delta)$ yields a presentation of the associated Coxeter group $W(\Delta)$ of type $\Delta$. We denote $\rho_{\Delta}: G(\Delta) \rightarrow W(\Delta)$ the canonical quotient map obtained by the addition of these relations.

The following observations are true for all Artin groups and were proved in [15]. If $T$ is a full subgraph of $\Delta$ then the subgroup of $G(\Delta)$ generated by the vertices of $T$ is canonically isomorphic to $G(T)$. Such subgroups shall be called standard parabolic. Moreover, the intersection of two standard parabolic subgroups of an Artin group is again a standard parabolic subgroup. Thus, for example, if $e, f \in E(\Delta)$ then $G(e) \cap G(f)=G(e \cap f)$, which is either the cyclic group $\langle s\rangle$ in the case that $e$ and $f$ share a common vertex $s$, or the trivial group (in the case $e$ and $f$ are disjoint). The analogous statements also hold for Coxeter groups.

Definition (CLTTF Artin group) The main Theorems in this paper shall apply to Artin (and Coxeter) groups whose defining graph satisfies the following conditions:

(C) $\Delta$ is connected and has at least 3 vertices;

(LT) all labels $m_{e}$, for $e \in E(\Delta)$, are at least 3; and

(TF) $\Delta$ has no triangles (no simple circuits of length 3 ).

If $\Delta$ satisfies all three of the above conditions then we refer to it as a CLTTF defining graph and we refer to $G(\Delta)$ as a CLTTF Artin group, and to $W(\Delta)$ as a CLTTF Coxeter group.

Conditions (LT) and (TF) correspond to what are known as the large type and triangle free conditions, either of which implies that the Artin group has cohomological (or geometric) dimension 2. The triangle free Artin groups are

\footnotetext{
${ }^{1}$ Our notion of defining graph differs from the frequently used "Coxeter graph" where, by contrast, the absence of an edge between $s$ and $t$ indicates a commuting relation $\left(m_{s t}=2\right)$ and the label $m_{s t}=\infty$ is used to designate the absence of a relation between $s$ and $t$. In our convention the label $\infty$ is never used.
} 
exactly the 2-dimensional, so-called, "FC type" Artin groups. The condition (C) simply serves to rule out the 2-generator or "dihedral type" Artin groups which are best treated as a separate case (see [12] for a treatment of their automorphism groups), as well as those Artin groups which are proper free products. (Using the Kurosh Subgroup Theorem it can be shown that an arbitrary Artin group $G(\Delta)$ is freely indecomposable if and only if $\Delta$ is connected).

Theorem 1 Let $\mathcal{G}$ denote the set of all CLTTF defining graphs (up to labelled graph isomorphism) and write Iso $(\mathcal{G})$ for the category (a groupoid) with objects $\mathcal{G}$ and morphisms the set of all isomorphisms $G(\Delta) \rightarrow G\left(\Delta^{\prime}\right)$ where $\Delta, \Delta^{\prime} \in \mathcal{G}$. Then $\operatorname{Iso}(\mathcal{G})$ is generated by the isomorphisms of type (1)-(4) listed below.

For the following definitions we make no assumptions on the defining graph $\Delta$. We first describe three classes of automorphisms.

(1) Graph automorphisms - Aut $(\Delta)$

Any label preserving graph automorphism of $\Delta$ induces in an obvious way an automorphism of $G(\Delta)$. We denote by $A u t(\Delta)$ the group of all such automorphisms.

(2) Inversion automorphisms $-\operatorname{Inv}(\Delta)$

These include the involution $\epsilon: G(\Delta) \rightarrow G(\Delta)$ such that $\epsilon(s)=s^{-1}$ for all $s \in V(\Delta)$, which we shall refer to as the global inversion of $G(\Delta)$, as well as the following involutions which we shall refer to as leaf inversions. For any edge $e=\{s, t\} \in E(\Delta)$ where $t$ is a terminal vertex and $m_{e}$ is even, we define the involution $\mu_{e}: G(\Delta) \rightarrow G(\Delta)$ by setting $\mu_{e}(t)=(s t s)^{-1}$ and $\mu_{e}(v)=v$ for all $v \in V(\Delta) \backslash\{t\}$. The global and leaf inversions together generate a subgroup of $\operatorname{Aut}(G(\Delta))$ isomorphic to $(\mathbb{Z} / 2 \mathbb{Z})^{l+1}$, where $l$ denotes the number of even labelled terminal edges in $\Delta$. We shall denote this subgroup by $\operatorname{Inv}(\Delta)$.

(3) Inner and Dehn twist automorphisms - Pure $(\Delta)$

Let $T$ denote an edge or vertex of $\Delta$ and suppose that $\Delta=\Delta_{1} \cup_{T} \Delta_{2}$ (by which we imply that $\Delta_{1}, \Delta_{2}$ are full subgraphs of $\Delta$ such that $\Delta_{1} \cup \Delta_{2}=\Delta$ and $\left.\Delta_{1} \cap \Delta_{2}=T\right)$. Let $g \in C_{G}(G(T))$ be an element of the centralizer of $G(T)$. Then we may define an automorphism of $G$ by setting

$$
\varphi(v)=g v g^{-1} \text { if } v \in V\left(\Delta_{1}\right), \text { and } \varphi(v)=v \text { if } v \in V\left(\Delta_{2}\right) .
$$

Such automorphisms shall be called Dehn twist automorphisms (along $T)$. We define Pure $(\Delta)$ to be the subgroup of $\operatorname{Aut}(G(\Delta))$ generated by the Dehn twist automorphisms. Note that putting $\Delta_{2}=T=\{s\}$ 
we obtain the inner automorphism 'conjugation by $s$ ' as a Dehn twist automorphism. Thus, Pure $(\Delta)$ contains the group $\operatorname{Inn}(G(\Delta))$ of inner automorphisms of $G(\Delta)$. By a nondegenerate Dehn twist we mean one which is not just an inner automorphism, namely a Dehn twist along a separating edge or vertex.

Note that each nondegenerate Dehn twist is defined in terms of a "visual splitting" of the Artin group, a decomposition as an amalgamated free product of standard parabolic subgroups, namely

$$
G(\Delta)=G\left(\Delta_{1}\right) \star_{G(T)} G\left(\Delta_{2}\right) .
$$

The global and leaf inversions respect any (proper) visual splitting of the group while the graph automorphisms carry any visual splitting to a similar one. Thus graph automorphisms and inversions of $G(\Delta)$ conjugate Dehn twist automorphisms to Dehn twist automorphisms. Moreover, the graph automorphisms preserve the set of even labelled terminal edges and therefore act by conjugation on the inversions. Thus $\operatorname{Aut}(G(\Delta))$ contains a subgroup of the form

$$
\operatorname{Pure}(\Delta) \rtimes \operatorname{Inv}(\Delta) \rtimes \operatorname{Aut}(\Delta) .
$$

Remark If $e=\{s, t\} \in E(\Delta)$ and $m_{e} \geq 3$ then the group $G(e)$ has infinite cyclic centre generated by the element $z_{e}=(s t)^{k}$ where $k=\operatorname{lcm}\left(m_{e}, 2\right) / 2$. We also define the element

$$
x_{e}=\underbrace{s t s t s \cdots}_{m_{e}} \text {. }
$$

This element generates the quasi-centre of $G(e)$, the subgroup of elements which leave the generating set $\{s, t\}$ invariant by conjugation. We have $z_{e}=x_{e}^{2}$ if $m_{e}$ is odd and $z_{e}=x_{e}$ if $m_{e}$ is even.

In the case where $G=G(\Delta)$ is a large type (LT) Artin group we can explicitly describe the centralizers of separating edges and vertices. If $e \in E(\Delta)$ then $C_{G}(G(e))=Z(G(e))=\left\langle z_{e}\right\rangle$. The centralizer of a generator $s \in V(\Delta)$ is the direct product of $\langle s\rangle$ with a (typically non-cyclic) free group of finite rank. A generating set for this free group may be obtained by observing that $C_{G}(\langle s\rangle) /\langle s\rangle$ is isomorphic to the vertex group at $s \in V(\Delta)$ in the groupoid with object set $V(\Delta)$ and generated by arrows $x_{e}: r \rightarrow r^{\prime}$ where $e=\{r, t\}$ and $r^{\prime}=x_{e} r x_{e}^{-1}$ ( $r^{\prime}=r$ if $m_{e}$ is even, and $t$ otherwise). We refer the reader to [13], or [14], for a more detailed description.

(4) Edge twist isomorphisms

Suppose that $\Delta=\Delta_{1} \cup_{e} \Delta_{2}$ where $e$ is a separating edge whose label $m_{e}$ 
is odd. Let $\Delta^{\prime}$ denote the labelled graph obtained by gluing $\Delta_{1}$ and $\Delta_{2}$ together along the edge $e$ where the identification map reverses the edge. Then we may define an isomorphism

$$
\varphi: G(\Delta) \rightarrow G\left(\Delta^{\prime}\right)
$$

by setting

$$
\varphi(v)=x_{e} v x_{e}^{-1} \text { if } v \in V\left(\Delta_{1}\right), \text { and } \varphi(v)=v \text { if } v \in V\left(\Delta_{2}\right) .
$$

We shall call such an isomorphism an edge twist, and we say that $\Delta$ and $\Delta^{\prime}$ are twist equivalent graphs. This generates an equivalence relation on the set of all defining graphs. (The collection $\mathcal{G}$ of all CLTTF defining graphs is invariant under twist equivalence). Note that in the case where $e=\{s, t\}$ and $t$ is a terminal vertex of $\Delta_{1}$, then $s$ is a separating vertex and we may think of $\Delta$ as the union of $\Delta_{1}^{\prime}:=\Delta_{1} \backslash e$ and $\Delta_{2}$ joined at the vertex $s$. In this case the edge twist $\varphi$ modifies the graph $\Delta$ by sliding the component $\Delta_{1}^{\prime}$ along the edge $e$ so that it is attached to $\Delta_{2}$ at the vertex $t$, instead of at $s$.

Remark The edge twist isomorphism described here is a special case of the "diagram twist" isomorphisms between Artin (and Coxeter) groups first described by Brady, McCammond, Mühlherr and Neumann in [4. The notion of (diagram) twist equivalence as introduced in [4] is defined, more generally, over the family of all defining graphs and there is considerable evidence for the conjecture that it is essentially this equivalence relation which classifies all Coxeter groups up to isomorphism. A recent survey of the isomorphism problem for Coxeter groups has been written by Mühlherr 17 .

Definition (Twist equivalence groupoid) Denote Biject $(\mathcal{G})$ the groupoid with object set $\mathcal{G}=\{$ CLTTF defining graphs $\}$ and a morphism $f: \Delta \rightarrow \Delta^{\prime}$ for each bijection $f: E(\Delta) \rightarrow E\left(\Delta^{\prime}\right)$ of the edge sets. Observe that every edge twist and every graph automorphism is naturally associated with a morphism in $\operatorname{Biject}(\mathcal{G})$. We define $\operatorname{Twist}(\mathcal{G})$ to be the subgroupoid of $\operatorname{Biject}(\mathcal{G})$ generated by the edge twists and graph automorphisms.

It is known that in any 2-dimensional Artin group the subgroups $\left\langle z_{e}\right\rangle$, for $e \in E(\Delta)$, are mutually non-conjugate (this may be readily seen from the action of $G(\Delta)$ on its Deligne complex, as described in Section 11). Thus the bijection $\bar{\varphi} \in \operatorname{Biject}(\mathcal{G})$ induced by any edge twist or graph automorphism $\varphi$ is determined by the action of $\varphi$ on the set of conjugacy classes of the cyclic subgroups $\left\langle z_{e}\right\rangle$ for $e \in E(\Delta)$. Note also that any element of $\operatorname{Pure}(\Delta) \rtimes \operatorname{Inv}(\Delta)$ 
acts trivially on this set. The following statement is largely a consequence of Theorem 1 and the above discussion.

Theorem 2 There exists a unique well-defined groupoid homomorphism

$$
\pi: \operatorname{Iso}(\mathcal{G}) \rightarrow \operatorname{Twist}(\mathcal{G})
$$

such that, writing $\pi(\varphi)=\bar{\varphi}$, we have $\left\langle z_{\bar{\varphi}(e)}\right\rangle \sim \varphi\left(\left\langle z_{e}\right\rangle\right)$ for all $e \in E(\Delta)$. The image of $\pi$ is Twist $(\mathcal{G})$ and the kernel at $\Delta \in \mathcal{G}$ is given by

$$
\operatorname{ker}(\pi, \Delta)=\operatorname{Pure}(\Delta) \rtimes \operatorname{Inv}(\Delta) .
$$

In particular, for fixed $\Delta \in \mathcal{G}$, the automorphism group of $G(\Delta)$ is a (finite) extension of Pure $(\Delta) \rtimes \operatorname{Inv}(\Delta)$ by a subgroup of $\operatorname{Sym}(E(\Delta))$ which consists of those permutations of $E(\Delta)$ obtained by composing edge twists and label preserving graph automorphisms. Moreover, two CLTTF Artin groups are isomorphic if and only if their defining graphs lie in the same connected component of $\operatorname{Twist}(\mathcal{G})$, ie, if and only if their defining graphs are twist equivalent.

Note that the connected components of the groupoids $\operatorname{Iso}(\mathcal{G})$, and Twist $(\mathcal{G})$ alike, correspond to the isomorphism classes of CLTTF Artin groups. Moreover, the connected components of $T$ wist $(\mathcal{G})$ are finite, and easily computable. Thus, as well as determining the automorphism group of any CLTTF Artin group, the above Theorem also solves the problem of classifying these groups up to isomorphism. In the language of [4, CLTTF Artin groups are "rigid up to diagram twisting". Note that spherical type Artin groups (those whose associated Coxeter groups are finite) are also known to be diagram rigid. This was recently shown by Paris in 20. Diagram rigidity is also known for right-angled Artin groups (the case where all edge labels in $\Delta$ are equal to 2 ) by the work of Droms [11. Other partial results on diagram rigidity appear in [4].

Example (No separating edges or vertices) Restricting our attention to those CLTTF Artin groups $G=G(\Delta)$ where $\Delta$ has no separating edge or vertex, we see that two such groups are isomorphic if and only if their defining graphs are isomorphic, and that

$$
\operatorname{Aut}(G)=\operatorname{Inn}(G) \rtimes(\langle\epsilon\rangle \times \operatorname{Aut}(\Delta)) .
$$

This is simply because, with no separating edges or vertices, there are no leaf inversions, nondegenerate Dehn twists or edge twist isomorphisms. Note that we also have $\operatorname{Inn}(G) \cong G$, since any CLTTF Artin group $G$ has trivial centre.

A simple example of the above type is where $\Delta$ is the 1 -skeleton of a 3 -cube and all edge labels are 3. This defining graph also satisfies the vertex rigidity condition (VR) required by part (ii) of Theorem 3 below. 
Example (No separating vertices) When $\Delta$ has separating edges but no separating vertices, then the group $\operatorname{Pure}(\Delta)$ is generated by the inner automorphisms and the Dehn twists along separating edges.

A chunk of $\Delta$ is a maximal connected full subgraph of $\Delta$ which is not separated by the removal of any edge or vertex which is separating in $\Delta$ (see Section 7 for a more detailed definition). Thus if $\Delta$ has no separating vertices it is the union of, say, $N$ distinct chunks glued along separating edges. Fixing a "base" chunk $B$, we may suppose that, up to an inner automorphism, each Dehn twist restricts to the identity on $G(B)$. It can be easily checked that the Dehn twists fixing $G(B)$ are mutually commuting elements. In this case we therefore have $\operatorname{Pure}(\Delta) \cong G \rtimes \mathbb{Z}^{N-1}$.

Example ( $\Delta$ a star graph) On the other hand, when there are separating vertices in $\Delta$ we expect the structure of $\operatorname{Aut}(G)$ to be somewhat more complicated. For example, one can check that when $\Delta$ is the star graph of $n+1$ vertices ( $n$ edges adjoined along a common vertex), and all edge labels are 3 say, then $\operatorname{Aut}(G)$ contains a subgroup isomorphic to the $n$-string braid group $B_{n}$. Let $e_{1}, . ., e_{n}$ denote the edges of $\Delta$ and, for $i=1, . ., n-1$, let $\sigma_{i}$ denote the automorphism of $G$ which is the product of the graph automorphism exchanging the edges $e_{i}$ and $e_{i+1}$ and the Dehn twist which conjugates the subgroup $G\left(e_{i}\right)$ by the element $z_{e_{i+1}}$. These automorphisms leave invariant the subgroup $F_{n}$ of $G$ which is freely generated by the set $\left\{z_{e}: e \in E(\Delta)\right\}$ (see Proposition 25), and they describe precisely the standard generators for Artin's representation of the braid group as a subgroup of $\operatorname{Aut}\left(F_{n}\right)$. (Moreover, one can check that elements of $B_{n}$ are represented by inner automorphism of $G$ if and only if they are central in the braid group. Thus $\operatorname{Out}(G)$ is not virtually abelian in this case).

\section{Abstract commensurators of Artin groups}

We recall that the abstract commensurator group $\operatorname{Comm}(\Gamma)$ of a group $\Gamma$ is defined to be the group of equivalence classes of isomorphisms between finite index subgroups of $\Gamma$, where two isomorphisms are considered equivalent if they agree on common finite index subgroup of their domains. Moreover, two

groups $\Gamma, \Gamma^{\prime}$ are said to abstractly commensurable if they possess finite index subgroups $H<\Gamma$ and $H^{\prime}<\Gamma^{\prime}$ which are isomorphic.

Theorem 3 Let $\Delta$ be a CLTTF defining graph with no separating edge or vertex. 
(i) If $G(\Delta)$ is abstractly commensurable to any CLTTF Artin group $G\left(\Delta^{\prime}\right)$ then $\Delta$ and $\Delta^{\prime}$ are label isomorphic.

(ii) Suppose moreover that $\Delta$ satisfies the following vertex rigidity condition: (VR) Any label preserving automorphism of $\Delta$ which fixes the neighbourhood of a vertex is the identity automorphism.

Then we have $\operatorname{Comm}(G)=\operatorname{Aut}(G) \cong G \rtimes(\langle\epsilon\rangle \times \operatorname{Aut}(\Delta))$.

With regard to part (i) of the above Theorem, we note that a 2-dimensional Artin group is not commensurable to any other Artin group which is not also 2-dimensional (since, for an Artin group, being 2-dimensional is equivalent to having $\mathbb{Z} \times \mathbb{Z}$ as a maximal rank abelian subgroup). We do not know whether the smaller class of CLTTF Artin groups is rigid in this sense.

Part (ii) of this Theorem should be compared with [8] where it is shown that $G$ is commensurable with its abstract commensurator group when $G$ belongs to one of the two infinite families of Artin groups of affine type $\widetilde{A}_{n}$ and $\widetilde{C}_{n}$, with $n \geq 2$. (The same holds for $G / Z$ where $G$ is an Artin group of finite type $A_{n}$ or $B_{n}$, with $n \geq 3$, and $Z$ denotes the infinite cyclic centre of $G$ ). In Section 11] we give an example of an abstract commensurator of a CLTTF Artin group $G(\Delta)$ which is not equivalent to an automorphism in the case where $\Delta$ has no separating edge or vertex, but fails to satisfy the condition (VR). This hypothesis is therefore necessary. Examples are also given of abstractly commensurable but non-isomorphic CLTTF Artin groups.

\section{Isomorphisms of Coxeter groups}

Finally we consider isomorphisms between Coxeter groups of CLTTF type. Let $I_{\text {so }_{W}}(\mathcal{G})$ denote the category (a groupoid) with objects $\mathcal{G}$ and morphisms the isomorphisms $W(\Delta) \rightarrow W\left(\Delta^{\prime}\right)$ for $\Delta, \Delta^{\prime} \in \mathcal{G}$. We note (by inspection of the isomorphisms of type (1)-(4)) that every isomorphism $\varphi: G(\Delta) \rightarrow G\left(\Delta^{\prime}\right)$ induces an isomorphism $\varphi_{W}: W(\Delta) \rightarrow W\left(\Delta^{\prime}\right)$. This is natural in the sense that $\varphi_{W} \circ \rho_{\Delta}=\rho_{\Delta^{\prime}} \circ \varphi$, where $\rho_{\Delta}: G(\Delta) \rightarrow W(\Delta): g \mapsto \bar{g}$ denotes the canonical surjection. Thus the mapping $\varphi \mapsto \varphi_{W}$ defines a groupoid homomorphism

$$
\rho: \operatorname{ISO}_{\mathrm{SO}}(\mathcal{G}) \rightarrow \operatorname{ISO}_{W}(\mathcal{G}) .
$$

Remark The above remarks imply, in particular, that the pure Artin group $P G(\Delta)$, which is defined as the kernel of the canonical quotient $\rho_{\Delta}: G(\Delta) \rightarrow$ $W(\Delta)$, is a characteristic subgroup of $G(\Delta)$ for CLTTF type Artin groups. 
This agrees with results already known for irreducible finite type Artin groups by Cohen and Paris [10] which generalised a much earlier Theorem of Artin [1] in the case of the braid groups.

There is a further source of Coxeter group automorphisms not induced from automorphisms of the associated Artin groups. These shall be thought of as "pure" automorphisms since, as with the inner and Dehn twist automorphism (induced from Pure $(\Delta)$ ), they respect the conjugacy class of the element $\bar{x}_{e}=$ $\rho_{\Delta}\left(x_{e}\right)$, for each $e \in E(\Delta)$.

Pure automorphisms of $W(\Delta)$ Let $e=\{s, t\} \in E(\Delta)$ denote a cut edge: every edge path in $\Delta$ from $s$ to $t$ passes through $e$. Then there are disjoint connected full subgraphs $\Delta_{1}, \Delta_{2}$ of $\Delta$ such that $\Delta=\Delta_{1} \cup e \cup \Delta_{2}$ with $\Delta_{1} \cap e=$ $\{s\}$ and $\Delta_{2} \cap e=\{t\}$. Let $m=m_{e} \geq 3$, and let $r \in \mathbb{N}$ such that $2 r+1$ is congruent $(\bmod m)$ to a unit in the $\operatorname{ring} \mathbb{Z} / m \mathbb{Z}$. Then we may define an automorphism of $W(\Delta)$ by setting

$$
\varphi(v)=(s t)^{r} v(s t)^{-r} \text { if } v \in V\left(\Delta_{1}\right), \text { and } \varphi(v)=v \text { if } v \in V\left(\Delta_{2}\right) .
$$

Such automorphisms shall be called dihedral twist automorphisms. We define $\operatorname{Pure}_{W}(\Delta)$ to be the subgroup of $\operatorname{Aut}(W(\Delta))$ generated by all dihedral twists, Dehn twists and inner automorphisms. In particular, $\operatorname{Pure}_{W}(\Delta)$ contains all automorphisms induced from $\operatorname{Pure}(\Delta)$.

The following Theorem gives a solution to the "classification" and "automorphism" problems for CLTTF Coxeter groups. We remark that the classification up to isomorphism is already contained in the work of Mühlherr and Weidmann [18. on reflection rigidity and reflection independance in large type (what they call "skew-angled") Coxeter groups. Also, the automorphism groups have already been determined in many of the cases covered here (and some besides) by Bahls [2]. The proof of Theorem 4 which we give consists in repeating the same sequence of arguments used to establish Theorems 11 and 2, with appropriate slight modification, in the context of Coxeter groups.

Theorem 4 The groupoid $\operatorname{IsoW}_{W}(\mathcal{G})$ is generated by pure automorphisms (elements of Pure $W(\Delta)$, for $\Delta \in \mathcal{G})$, graph automorphisms and edge twist isomorphisms. More precisely, there is a surjective groupoid homomorphism

$$
\pi_{W}: \operatorname{IsO}_{W}(\mathcal{G}) \rightarrow \operatorname{Twist}(\mathcal{G})
$$

with $\pi_{W} \circ \rho=\pi$, and for each $\Delta \in \mathcal{G}$ we have

$$
\operatorname{ker}(\pi, \Delta)=\operatorname{Pure}_{W}(\Delta) .
$$


In particular, for fixed $\Delta \in \mathcal{G}$, the automorphism group of $W(\Delta)$ is a (finite) extension of $\operatorname{Pure}_{W}(\Delta)$ by the subgroup of $\operatorname{Sym}(E(\Delta))$ appearing as a vertex group in Twist $(\mathcal{G})$. Moreover, two CLTTF Coxeter groups are isomorphic if and only if their defining graphs lie in the same connected component of Twist $(\mathcal{G})$, ie, if and only if their defining graphs are twist equivalent.

The automorphism group of a CLTTF Coxeter group has previously been described by Patrick Bahls 2] under the added hypotheses that all edge labels are even and the defining graph cannot be separated into more than 2 components by removal of a single edge. In fact, in his work, Bahls does not suppose that the defining graph is triangle free, and so treats many cases which are not covered here. He also gives several statements (see Corollaries 1.2, 1.3, 1.4 of 2] ) giving further details on the size and structure of $\operatorname{Out}(W)$ which probably extend to the CLTTF case.

As an example, consider the case where $\Delta$ has no separating vertices. In this case there are no dihedral twists and $\operatorname{Pure}_{W}(\Delta) \cong W(\Delta) \rtimes(\mathbb{Z} / 2 \mathbb{Z})^{R-1}$, where $R$ is the number of distinct maximal full subgraphs of $\Delta$ not separated by any even labelled edge (compare with Corollary 1.3 in [2]). In particular, Out $(W)$ is finite in this case. Note, however, that the corresponding Artin groups have typically infinite outer automorphism groups. In the case of no separating vertices we have already seen that $\operatorname{Pure}(\Delta) \cong G(\Delta) \rtimes(\mathbb{Z})^{N-1}$ with $N \geq R$.

Recently, Mühlherr and Weidmann [18, have proved results on reflection rigidity and reflection independance in the wider class of large type (LT) Coxeter groups which give the same solution to the classification problem as given by Theorem 4 above. We note that Bahls [3] has also obtained a similar classification for those Coxeter groups having 2-dimensional Davis complex (equivalently, those associated to 2-dimensional Artin groups). Several other results in this direction are discussed in the survey by Mühlherr [17. It seems reasonable to conjecture that Theorems 10 2 and 4 all hold unchanged over the class of connected large type (CLT) defining graphs, and that similar results might also hold for all 2-dimensional Artin groups, or for general Coxeter groups.

Acknowledgement This work has benefitted from discussions with many people. In particular, I would like to thank Benson Farb, Luisa Paoluzzi, Bernhard Mühlherr, Patrick Bahls, Gilbert Levitt and the referee for a variety of helpful suggestions and comments. 


\section{The Deligne complex $\mathbb{D}$}

For simplicity, we formulate the following definitions only in the case where the Artin group $G=G(\Delta)$ is 2 -dimensional, equivalently, where every triangle in $\Delta$ with edge labels $m, n, p$ satisfies $1 / m+1 / n+1 / p \leq 1$. See [9] for details of the general construction.

Definition of the Deligne complex $\mathbb{D}$ Let $K$ denote the geometric realisation of the derived complex of the partially ordered set

$$
\left\{V_{\emptyset}\right\} \cup\left\{V_{s}: s \in V(\Delta)\right\} \cup\left\{V_{e}: e \in E(\Delta)\right\},
$$

where the partial order is given by setting $V_{\emptyset}<V_{s}$ for all $s \in V(\Delta)$, and $V_{s}<V_{e}$ whenever $s$ is a vertex of the edge $e$. Thus $K$ is a finite 2-dimensional simplicial complex. We may also view $K$ as a squared complex with one square cell for each edge of $\Delta$. If $e=\{s, t\} \in E(\Delta)$ then the corresponding square cell has vertices $V_{\emptyset}, V_{s}, V_{t}, V_{e}$. We note that, viewing $K$ as a squared complex in this way we have $\operatorname{Lk}\left(V_{\emptyset}, K\right) \cong \Delta$. See Figure 1.

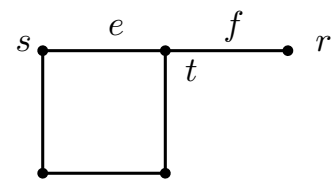

$\Delta$

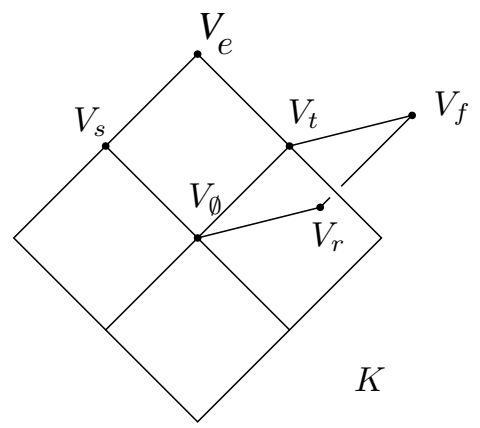

Figure 1: Defining graph $\Delta$ and squared complex $K$ for a 2-dimensional Artin group

Let $\mathcal{K}$ denote the complex of groups with underlying complex $K$ and vertex groups $G\left(V_{\emptyset}\right)=\{1\}, G\left(V_{s}\right)=\langle s\rangle=G(s)$, for $s \in V(\Delta)$, and $G\left(V_{e}\right)=G(e)$, for $e \in E(\Delta)$. Then $\mathcal{K}$ is a developable complex of groups (cf [9]) whose fundamental group is the Artin group: $\pi_{1}(\mathcal{K})=G(\Delta)$.

Definition (Deligne complex) Let $G=G(\Delta)$ be a 2-dimensional Artin group. We define the Deligne complex $\mathbb{D}$, of type $\Delta$, to be the universal covering $\widetilde{\mathcal{K}}$ of the complex of groups $\mathcal{K}$ just described, equipped with the action of $G$ by covering transformations. 
The Artin group acts by simplicial isomorphisms of $\mathbb{D}$ with vertex stabilizers either trivial or conjugate to one of the standard parabolic subgroups $G(s)$, for $s \in V(\Delta)$, or $G(e)$, for $e \in E(\Delta)$. We classify the vertices of $\mathbb{D}$ into three kinds according to their stabilizers:

Rank 0 vertices of the form $g V_{\emptyset}$ for $g \in G$. These have trivial stabilizer.

Rank 1 vertices $g V_{s}$ for $s \in V(\Delta)$ and $g \in G-\operatorname{Stab}\left(g V_{s}\right)=g\langle s\rangle g^{-1}$.

Rank 2 vertices $g V_{e}$ for $e \in E(\Delta)$ and $g \in G-\operatorname{Stab}\left(g V_{e}\right)=g G(e) g^{-1}$.

Note that every point in the open neighbourhood of a rank 0 vertex represents a free orbit of the group action (since the group action is strictly cellular).

We also note that an analogous construction replacing the vertex groups of $\mathcal{K}$ with the corresponding finite standard parabolic subgroups of the Coxeter group $W$ results in a description of the Davis complex, which we shall denote by $\mathbb{D}_{W}$. There is a natural simplicial map $p_{W}: \mathbb{D} \rightarrow \mathbb{D}_{W}$ induced by the canonical projection $G \rightarrow W$ and an inclusion $i_{W}: \mathbb{D}_{W} \hookrightarrow \mathbb{D}$ induced by the Tits section $W \hookrightarrow G$. We have $p_{W} \circ i_{W}$ equal to the identity on $\mathbb{D}_{W}$.

Definition (Metrics on $\mathbb{D}$ ) There are two natural choices of $G$-equivariant piecewise Euclidean metric for the complex $\mathbb{D}$. The first, and perhaps most natural, is known as the Moussong metric and is defined such that, for $e=$ $\{s, t\} \in E(\Delta)$, the simplex $\left(V_{\emptyset}, V_{s}, V_{e}\right)$ is a Euclidean triangle with angles $\frac{\pi}{2}$ at $V_{s}$ and $\frac{\pi}{2 m_{e}}$ at $V_{e}$. (See [16], also 9]). The Moussong metric on $\mathbb{D}$ is known to be $\operatorname{CAT}(0)$ for all 2-dimensional Artin groups. This property will be used in Section 3

The second is the cubical metric obtained by viewing $\mathbb{D}$ as a squared complex (as in Figure 1) built from unit Euclidean squares. For $G(\Delta) 2$-dimensional, the cubical metric on $\mathbb{D}$ is known to be $\operatorname{CAT}(0)$ if and only if $\Delta$ is triangle free (see [9]). In particular, this metric is $\operatorname{CAT}(0)$ in the CLTTF case. The cubical metric shall be used in Section [6]

We note that each of these metrics induces a unique metric on the Davis complex $\mathbb{D}_{W}$ such that the map $i_{W}: \mathbb{D}_{W} \hookrightarrow \mathbb{D}$ is an isometric embedding.

The following definition and lemma will be relevant in Section 3 ,

Definition (Hyperbolic type) We shall say that a defining graph $\Delta$, or the associated Artin group $G(\Delta)$, is of hyperbolic type if the Coxeter group $W(\Delta)$ is a Gromov hyperbolic group. Equivalently, $\Delta$ is of hyperbolic type if and only if the Davis complex $\mathbb{D}_{W}$ is a $\delta$-hyperbolic metric space with respect to either 
the Moussong metric or the cubical metric. (This is because the Coxeter group acts properly and co-compactly by isometries with respect to either metric on the Davis complex and so is quasi-isometric to $\mathbb{D}_{W}$ ).

Lemma 5 Let $G(\Delta)$ be a 2-dimensional Artin group. Then the following are equivalent

(1) $G(\Delta)$ (or $\Delta$ ) is of hyperbolic type;

(2) the Deligne complex $\mathbb{D}$ equipped with the Moussong metric is a $\delta$ hyperbolic metric space;

(3) $\Delta$ contains no triangle having edge labels $m, n, p$ with $1 / m+1 / n+1 / p=1$ and no square with all edge labels equal to 2 .

Proof We suppose throughout that the Deligne complex $\mathbb{D}$ is equipped with the Moussong metric. Since $G(\Delta)$ is 2 -dimensional, this implies that $\mathbb{D}$ is a CAT(0) space. By the Flat Plane Theorem (see [7]) this space is $\delta$-hyperbolic if and only if it contains no isometrically embedded flat plane $\mathbb{E}^{2}$. If such a plane existed in $\mathbb{D}$, it would necessarily be a simplicial subcomplex and so contain at least one rank 0 vertex. Morever, it would contribute a simple circuit of length exactly $2 \pi$ to the link of any such vertex. We note that the link of a rank 0 vertex of $\mathbb{D}$ contains a circuit of length exactly $2 \pi$ if and only if there exists either a triangle in $\Delta$ with labels $m, n, p$ such that $1 / m+1 / n+1 / p=1$, or a square in $\Delta$ with all labels 2 . Thus, condition (3) implies that no embedded flat plane can occur in $\mathbb{D}$, and hence that (2) holds. On the other hand if (3) fails then $W(\Delta)$ contains either a Euclidean triangle group, or $D_{\infty} \times D_{\infty}$. In either case $W(\Delta)$ contains a subgroup $\mathbb{Z} \times \mathbb{Z}$, and so cannot be Gromov hyperbolic. Thus, we have shown (1) implies (3), as well as (3) implies (2).

Finally, we use the fact that the Davis complex $\mathbb{D}_{W}$ (with the Moussong metric) embeds isometrically in $\mathbb{D}$. Any flat plane in $\mathbb{D}_{W}$ is therefore also a flat plane in $\mathbb{D}$. Thus, by the Flat Plane Theorem, $\mathbb{D}_{W}$ is $\delta$-hyperbolic if $\mathbb{D}$ is, and so (2) implies (1).

We note that any CLTTF Artin group is necessarily a 2-dimensional Artin group of hyperbolic type. Similarly, any CLTTF Coxeter group has 2-dimensional $\delta$-hyperbolic Davis complex.

In Section 3 we shall also use the following statement which is a consequence of a quite general result due to Bridson [6]. We recall that an isometry $\gamma$ of a geodesic metric space $X$ is said to be semi-simple if it attains its translation 
length: $|\gamma|:=\inf \{d(x, \gamma x): x \in X\}$ is realised at some point in $X$. Semisimple elements are classified into two classes: elliptic if $|\gamma|=0$; and hyperbolic if $|\gamma| \neq 0$. Bridson's result in [6] states that any isometry of a geodesic metric simplicial complex having finitely many isometry types of cells is necessarily semi-simple. As a consequence we have:

Lemma 6 Let $G$ be a 2-dimensional Artin group. Then the action of $G$ on $\mathbb{D}$ is semi-simple (with respect to either the Moussong metric, or the cubical metric).

\section{Structure of vertex stabilisers and fixed sets in $\mathbb{D}$}

We consider the 2-generator Artin groups which appear as the stabilizers of rank 2 vertices of the Deligne complex $\mathbb{D}$ associated to a 2-dimensional Artin group and derive some basic properties which will be useful in the sequel. Using one of these properties, we also give a classification of the fixed sets in $\mathbb{D}$ for arbitrary elements of a 2-dimensional Artin group.

Recall that if $e=\{s, t\} \in E(\Delta)$ with label $m_{e}$ then the group $G(e)$ is given by the presentation

$$
G(e)=\langle s, t \mid \underbrace{s t s t s \cdots}_{m_{e}}=\underbrace{t s t s t \cdots}_{m_{e}}\rangle
$$

When $m_{e} \geq 3$ the centre of $G(e)$ is infinite cyclic generated by the element $z_{e}:=$ $(s t)^{k}$ where $k=m_{e}$ if $m_{e}$ is odd, and $k=m_{e} / 2$ if $m_{e}$ is even. (Alternatively $k=\operatorname{lcm}\left(m_{e}, 2\right) / 2$.) We wish to consider the quotient of $G(e)$ by its centre, which we shall denote by

$$
\Gamma=G(e) /\left\langle z_{e}\right\rangle .
$$

We shall systematically write $\bar{x}$ for the image in $\Gamma$ of an element $x \in G(e)$.

We note that $\Gamma$ is a virtually free group (and virtually cyclic if and only if $\left.m_{e}=2\right)$. In fact,

$$
\Gamma \cong\left\{\begin{aligned}
\mathbb{Z}_{2} \star \mathbb{Z}_{k} & \text { if } m_{e} \text { odd } \\
\mathbb{Z} \star \mathbb{Z}_{k} & \text { if } m_{e} \text { even }
\end{aligned}\right.
$$

The free factors here are generated by the elements $\overline{s t}$ of order $k=1 \mathrm{~cm}\left(m_{e}, 2\right) / 2$ and either $\bar{x}_{e}$ of order 2 when $m_{e}$ is odd, or $\bar{s}$ of infinite order in the case $m_{e}$ even.

It is clear from the the above description that, in each case, $\Gamma$ admits a proper co-compact action on a regular $k$-valent metric tree $T$ (with edge lengths equal 
to 1) where the fixed set of any elliptic element consists of a single point elements conjugate to $\overline{s t}$ fix the vertices and, in the case $m_{e}$ odd, elements conjugate to $\bar{x}_{e}$ fix the midpoints of edges. On the other hand, both generators $s$ and $t$ of $G(e)$ act by hyperbolic isometries of $T$ of translation length 1 . (These actions are described in more detail in Section 2 of [5], for example).

Finally, we note that any Artin group $G(\Delta)$ admits a standard length homomorphism $\ell: G(\Delta) \rightarrow \mathbb{Z}$ defined by setting $\ell(s)=1$ for all $s \in V(\Delta)$.

Lemma 7 Let $G(e)$ be the rank 2 Artin group associated to an edge $e=\{s, t\}$, with label $m_{e} \geq 2$. Let $R$ denote the set of all elements conjugate in $G(e)$ into the generating set $\{s, t\}$, and let $x \in G(e)$. Then

(i) $C_{G(e)}(\langle x\rangle)$ is virtually abelian if and only if either $m_{e}=2$ or $m_{e} \geq 3$ and $x$ is not central.

(ii) Let $u \in R$ and $k \in \mathbb{Z} \backslash\{0\}$. Then $C_{G(e)}\left(\left\langle u^{k}\right\rangle\right)=\left\langle u, z_{e}\right\rangle \cong \mathbb{Z} \times \mathbb{Z}$, and if $x^{k}=u^{k}$ then $x=u$.

(iii) Suppose $m_{e} \geq 3$. Let $u, v \in R$ and $k, l \in \mathbb{Z} \backslash\{0\}$. If $u^{k}$ and $v^{l}$ commute then $u=v$.

Proof We shall suppose throughout that $m_{e} \geq 3$, the case where $m_{e}=2$ and $G(e) \cong \mathbb{Z}^{2}$ being easily checked.

(i) If $x$ lies in the centre $Z(G(e))$ then $C_{G(e)}(\langle x\rangle)=G(e)$ which is not virtually abelian (since $m_{e} \geq 3$ ). On the other hand, if $x \notin Z(G(e))$ then its image $\bar{x}$ in $\Gamma$ is nontrivial. We consider the action of $\bar{x}$ on the tree $T$. If $\bar{x}$ is elliptic then its fixed set consists of a single point $p$. But then $C_{\Gamma}(\bar{x})$ fixes $p$, so must be finite. If $\bar{x}$ is hyperbolic then $C_{\Gamma}(\bar{x})$ leaves invariant its axis. In either case $\bar{x}$ generates a finite index subgroup of $C_{\Gamma}(\bar{x})$. Therefore $x$ and $z_{e}$ generate a finite index abelian subgroup of $C_{G(e)}(x)$.

(ii) Since $u$ is conjugate to a generator, $\bar{u}$ is hyperbolic on $T$ with translation length $|\bar{u}|=1$. Let $A \subset T$ denote the translation axis for $\bar{u}$. This is also the unique translation axis for each power of $\bar{u}$. Let $x \in C_{G(e)}\left(\left\langle u^{k}\right\rangle\right)$. Then, since $\bar{x}$ commutes with $\bar{u}^{k}$, it leaves invariant the axis $A$ (without reversing its direction). Since $\bar{u}$ has unit translation length we can find $n \in \mathbb{Z}$ such that $\bar{x}$ and $\bar{u}^{n}$ differ by an elliptic fixing the whole axis $A$ and, since the fixed set of any elliptic in $\Gamma$ is a single point in $T$, we have that $\bar{x}=\bar{u}^{n}$. It follows that $x \in\left\langle u, z_{e}\right\rangle$. Thus $C_{G(e)}\left(\left\langle u^{k}\right\rangle\right)=\left\langle u, z_{e}\right\rangle \cong \mathbb{Z} \times \mathbb{Z}$. 
If $x^{k}=u^{k}$ then $x$ centralizes $u^{k}$, and so $x \in\left\langle u, z_{e}\right\rangle \cong \mathbb{Z} \times \mathbb{Z}$. But now we have $x=u$ since uniqueness of roots holds in a free abelian group.

(iii) Since they are conjugate to generators, $u$ and $v$ project to hyperbolic isometries $\bar{u}$ and $\bar{v}$ of $T$ with translation length 1 in each case. If $u^{k}$ and $v^{l}$ commute for nonzero $k$ and $l$ then $\bar{u}$ and $\bar{v}$ must also share an axis in $T$. But then $\bar{u}=\bar{v}^{ \pm 1}$, and one of $u v^{-1}$ or $u v$ lies in the centre $\left\langle z_{e}\right\rangle$. But since $\ell\left(z_{e}\right)=\operatorname{lcm}\left(m_{e}, 2\right) \geq 3$, while $\ell(u)=\ell(v)=1$ we deduce that $u=v$.

We now consider the action of a 2-dimensional Artin group $G=G(\Delta)$ on its Deligne complex $\mathbb{D}$.

Definition (Fixed sets and $F_{s}$ ) For $g \in G$ we write $\operatorname{Fix}(g)$ for the (possibly empty) set of points in $\mathbb{D}$ left fixed by $g$. If $s \in V(\Delta)$ we write $F_{s}$ for the fixed set $\operatorname{Fix}(s)$ of $s$.

Note that $F_{s}$ is necessarily a geodesically convex subcomplex of $\mathbb{D}$. Moreover, since rank 0 vertices have trivial stabilizer, $F_{s}$ lies in that part of the 1-skeleton of $\mathbb{D}$ which is spanned by rank 1 and 2 vertices. Consequently $F_{s}$ is a tree (since it is geodesically convex) whose vertices are alternately rank 1 and 2 vertices of $\mathbb{D}$.

Lemma 8 Suppose that $G=G(\Delta)$ is a 2-dimensional Artin group, and let $x \in G \backslash\{1\}$.

(i) If $x \in\langle s\rangle$, for $s \in V(\Delta)$, then $\operatorname{Fix}(x)=F_{s}$.

(ii) If $x \in G(e)$, for $e=\{s, t\} \in E(\Delta)$, but $x$ is not conjugate in $G(e)$ into $\langle s\rangle$ or $\langle t\rangle$, then $\operatorname{Fix}(x)=\left\{V_{e}\right\}$.

(iii) If $x$ is not conjugate in $G$ to any of the elements covered by cases (i) and (ii) above, then $\operatorname{Fix}(x)=\emptyset$.

Proof (i) Let $x=s^{k}$ for some $k \neq 0$. Clearly $F_{s} \subset \operatorname{Fix}\left(s^{k}\right)$. If $\operatorname{Fix}\left(s^{k}\right) \neq F_{s}$ then there must be some edge $g\left[V_{t}, V_{e}\right]$ of $\mathbb{D}\left(g \in G, e=\left\{t, t^{\prime}\right\} \in E(\Delta)\right)$ which is fixed by $s^{k}$ but only one of whose vertices is fixed by $s$. If $s$ fixes $g V_{t}$ then it also fixes $g V_{e}$ (since $G(t)<G(e)$ ) so we may suppose that $s$ fixes $g V_{e}$ but not $g V_{t}$. Then, writing $y=g^{-1} s g$, we have $y \in G(e)$. On the other hand, since $s^{k}$ fixes $g V_{t}$ we have that $y^{k} \in\langle t\rangle$. Comparing lengths, we must have $y^{k}=t^{k}$ and therefore $y=t$, by Lemma 7 (ii). But then $s$ fixes the vertex $g V_{t}$ contrary to the choice of edge. Thus $\operatorname{Fix}\left(s^{k}\right)=F_{s}$. 
(ii) If $x \in G(e)$ then it clearly fixes the point $V_{e}$ in $\mathbb{D}$. If, however, $\operatorname{Fix}(x)$ contains any other vertex of $\mathbb{D}$ then it contains a neighbouring vertex, that is $g V_{t}$ or $g V_{s}$ for some $g \in G(e)$. But that is to say that $x$ is conjugate, in $G(e)$, into one of the subgroups $\langle s\rangle$ or $\langle t\rangle$.

(iii) If $\operatorname{Fix}(x) \neq \emptyset$ then $x$ must fix some rank 2 vertex (if it fixes a rank 1 vertex then it fixes every neighbouring rank 2 vertex). But then $x$ is conjugate to $x^{\prime} \in G(e)$ for some edge $e \in E(\Delta)$ and if $x^{\prime}$ is not covered by case (ii) it is conjugate to an element covered by case (i).

\section{CNVA subgroups and their fixed sets in $\mathbb{D}$}

Definition Let $C$ denote a nontrivial (necessarily infinite) cyclic subgroup of $G$. We say that $C$ is $C N V A$ ("centralizer not virtually abelian") in $G$ if its centralizer $C_{G}(C)$ is not virtually abelian.

Note that if $H$ is a finite index subgroup of $G$ and $C<H$, then $C$ is CNVA in $G$ if and only if it is CNVA in $H$ (ie $C_{H}(C)$ is not virtually abelian). The property of being CNVA is also inherited by subgroups of $C$, for if $C^{\prime}<C$ then the centralizer $C_{G}\left(C^{\prime}\right)$ contains $C_{G}(C)$ and so fails to be virtually abelian unless $C_{G}(C)$ is virtually abelian.

Definition (Internal vertex) Let $\Delta$ be an Artin defining graph. By an internal vertex of $\Delta$ we mean a vertex of valence at least two.

Lemma 9 Let $G=G(\Delta)$ be a 2-dimensional Artin group.

(i) If $e \in E(\Delta)$ with $m_{e} \geq 3$ then each nontrivial subgroup of $\left\langle z_{e}\right\rangle$ is CNVA.

(ii) If $s \in V(\Delta)$ is an internal vertex then each nontrivial subgroup of $\langle s\rangle$ is CNVA.

(iii) Suppose that $s \in V(\Delta)$ is not conjugate in $G(\Delta)$ to any generator corresponding to an internal vertex of $\Delta$. Then NO nontrivial subgroup of $\langle s\rangle$ is CNVA.

Proof (i) The fact that $C_{G}\left(\left\langle z_{e}\right\rangle\right)=G(e)$ is virtually nonabelian free by cyclic when $m_{e} \geq 3$ ensures that $\left\langle z_{e}\right\rangle$ (and each of its subgroups) is CNVA for all $e \in E(\Delta)$ with $m_{e} \geq 3$. 
(ii) Let $s \in V(\Delta)$. We consider the tree $F_{s}$ lying in the 1 -skeleton of $\mathbb{D}$ which is the fixed point set of $s$. This tree is left invariant by $C_{G}(\langle s\rangle)$, and we may therefore consider the action of the centralizer on $F_{s}$. We note that the rank 1 vertex $V_{s}$ lies in $F_{s}$, and that $\operatorname{Stab}\left(V_{s}\right)=G(s)=\langle s\rangle$. Any vertex of $\mathbb{D}$ which is adjacent to $V_{s}$ is $V_{e}$ for some $e \in E(\Delta)$ such that $s$ is a vertex of $e$.

Suppose now that $s$ is internal. Then there are rank 2 vertices $V_{e}$ and $V_{d}$ which lie in $F_{s}$, for distinct edges $e, d$ adjacent to $s$. (Note that the vertex $V_{s}$ lies midway between $V_{e}$ and $V_{d}$ ). The element $z_{e}$ (resp. $z_{d}$ ) centralizes $s$ and fixes $V_{e}$ (resp. $V_{d}$ ) but does not fix the point $V_{s}$. Since the elements $z_{e}$ and $z_{d}$ are acting in this way on a tree they necessarily generate a free group of rank 2 inside $C_{G}(\langle s\rangle)$, implying that $\langle s\rangle$ (and hence $\left\langle s^{k}\right\rangle$ for any $k \neq 0$ ) is CNVA.

(iii) We note that if $s$ belongs to an odd labelled edge $e=\{s, t\}$ then $s$ is conjugate to $t$ (by the element $x_{e}$ ). It follows that there are exactly three ways that $s$ can fail to be conjugate to an internal vertex generator (we have not supposed here that $\Delta$ is connected). Either

(a) $s$ is an isolated vertex of $\Delta$, or

(b) $s$ lies in a component of $\Delta$ which consists of a single edge $e$, or

(c) $s$ lies in a unique edge $e$, and $m_{e}$ is even.

We recall that, in general, the fixed set $F_{s}=\operatorname{Fix}(s)$ is a connected 1-dimensional subcomplex of $\mathbb{D}$, in fact a tree, whose vertices are alternately vertices of rank 1 and 2. Recall also that $F_{s}=\operatorname{Fix}\left(s^{k}\right)$, for all $k \geq 1$, by Lemma 8 (i). We use the basic fact that the centralizer of any element $g$ must leave invariant the set $\operatorname{Fix}(g)$. Thus $C_{G}\left(\left\langle s^{k}\right\rangle\right)$ leaves $F_{s}$ invariant, for all $k \geq 1$.

In case $(\mathrm{a}), F_{s}$ consists solely of the vertex $V_{s}$, since this vertex is not adjacent in $\mathbb{D}$ to any rank 2 vertex at all. In this case, $C_{G}\left(\left\langle s^{k}\right\rangle\right)$ must fix $V_{s}$ and is therefore an infinite cyclic group (since $\left.\operatorname{Stab}\left(V_{s}\right)=\langle s\rangle\right)$. Thus, in case (a), $\left\langle s^{k}\right\rangle$ fails to be CNVA, for all $k \geq 1$.

In cases (b) and (c) we claim that $F_{s}$ is a bounded (but still infinite) tree containing exactly one rank 2 vertex, namely the vertex $V_{e}$. First note that any rank 1 vertex of $F_{s}$ can be written $h V_{t}$ where $h \in G$ and $t \in V(\Delta)$ is a generator which is conjugate to $s$ (in fact we must have $s=h t h^{-1}$ because $\langle s\rangle \leq \operatorname{Stab}\left(h V_{t}\right)=h\langle t\rangle h^{-1}$ and $\left.\ell(s)=\ell(t)=1\right)$. Moreover, any edge of $F_{s}$ may be written $h\left[V_{t}, V_{f}\right]$ for some $h \in G$, some $t$ conjugate to $s$, and some $f \in E(\Delta)$ such that $t \in f$.

Now observe that, in both cases (b) and (c), there exists a homomorphism $\nu: G \rightarrow \mathbb{Z}$ such that $\nu(t)=0$ if $t$ lies in some edge different from $e$, and 
$\nu(t)=1$ otherwise. In particular $\nu(s)=1$, and clearly $\nu(t)=1$ for any generator $t$ which is conjugate to $s$. This shows that $e$ is the only edge which can possibly contain a vertex $t$ such that $s$ and $t$ are conjugate. It follows that every edge of $F_{s}$ is a translate of $\left[V_{t}, V_{e}\right]$ for some $t \in e$, and in particular that every rank 2 vertex is a translate of $V_{e}$. However, since each rank 1 vertex in $\mathbb{D}$ can be adjacent to at most one translate of a given rank 2 vertex, it now follows (by connectedness) that $F_{s}$ lies entirely in the neighbourhood of the vertex $V_{e}$.

Since it leaves $F_{s}$ invariant, we deduce in cases (b) and (c) that $C_{G}\left(\left\langle s^{k}\right\rangle\right)$ must fix $V_{e}$ (the unique rank 2 vertex of $F_{s}$ ), and hence is a subgroup of $G(e)$, for all $k \geq 1$. But then $\left\langle s^{k}\right\rangle$ is not CNVA since, by Lemma Z7(i), it has virtually abelian centralizer in $G(e)$.

Remark 10 It is implicit in the above proof that, for $s \in V(\Delta)$, the cyclic group $\langle s\rangle$ is CNVA if and only if its fixed set $F_{s}$ is an unbounded tree.

Lemma 11 Let $G=G(\Delta)$ be a 2-dimensional Artin group of hyperbolic type. A cyclic subgroup of $G$ is CNVA if and only if it is conjugate in $G$ to either a subgroup of $\left\langle z_{e}\right\rangle$ for some $e \in E(\Delta)$ with $m_{e} \geq 3$, or a subgroup of $\langle s\rangle$ for some internal vertex $s \in V(\Delta)$.

Proof By Lemma 9, it will suffice to show that any CNVA subgroup is conjugate into a subgroup of either $\left\langle z_{e}\right\rangle$, for some $e \in E(\Delta)$ with $m_{e} \geq 3$, or $\langle s\rangle$, for some $s \in V(\Delta)$.

We suppose for the purposes of this proof that $\mathbb{D}$ is equipped with the Moussong metric, and so is $\mathrm{CAT}(0)$ by [9. Suppose that $C$ is a CNVA cyclic subgroup of $G$ generated by the element $\gamma$. By Lemma 6 and the classification of semisimple isometries, this element is either elliptic or hyperbolic.

Assume firstly that $\gamma$ is elliptic. By Lemma 8, either $\gamma$ fixes some rank 1 vertex, and so is conjugate into $\langle s\rangle$ for some $s \in V(\Delta)$ as required, or $\operatorname{Fix}(\gamma)=\left\{g V_{e}\right\}$ for some $g \in G$ and $e \in E(\Delta)$. In the latter case the centralizer $C_{G}(\langle\gamma\rangle)$ must also fix the vertex $g V_{e}$ and so is a subgroup of $\operatorname{Stab}\left(g V_{e}\right)=g G(e) g^{-1}$. But then, by Lemma 7(i), it follows that $\gamma$ is an element of $g\left\langle z_{e}\right\rangle g^{-1}$ and $m_{e} \geq 3$, since otherwise it would have virtually abelian centralizer.

We now assume that $\gamma$ is hyperbolic. Let $M$ denote the minset of $\gamma$. Then by, Theorem II.6.8 of [7, $M \cong T \times \mathbb{R}$ where $T$ is, in our case, a metric tree. However, $T$ must be a bounded tree, since otherwise we would have a flat plane $\mathbb{E}^{2}$ isometrically embedded in $\mathbb{D}$, contradicting Lemma 5 (with the hypothesis that $\Delta$ is hyperbolic type). Therefore, $T$ has a fixed point $c$ under the action 
of $C_{G}(C)$ (cf Corollary II.2.8 of [7]). Thus $C_{G}(C)$ leaves invariant the $\gamma-$ axis $A_{c}=\{c\} \times \mathbb{R}$. Note that $A_{c}$ has a metric simplicial structure (induced from the structure on $\mathbb{D})$ with discrete automorphism group $\operatorname{Aut}\left(A_{c}\right)$. The group $C_{G}(C)$ acts via a homomorphism to $\operatorname{Aut}\left(A_{c}\right)$ whose kernel we denote $H$. Moreover the translation $\gamma$ acts co-compactly on the axis, so generates a finite index subgroup of $\operatorname{Aut}\left(A_{c}\right)$. It follows that $C_{G}(C)$ contains the product $H \times C$ with finite index. Note also that the only points in $\mathbb{D}$ which have nonabelian stabilizer are the rank 2 vertices. Since these form a discrete set, while the fixed set of $H$ contains a whole real line $A_{c}$, it follows that $H$ must be abelian (either trivial or infinite cyclic). But then $C_{G}(C)$ is virtually abelian, a contradiction.

\section{Abstract commensurators and the graph $\Theta$ of fixed sets in $\mathbb{D}$}

We recall briefly the definition of an abstract commensurator of groups. Given groups $\Gamma_{1}, \Gamma_{2}$, we define

$$
\operatorname{Comm}\left(\Gamma_{1}, \Gamma_{2}\right)=\left\{\varphi: H_{1} \cong H_{2}: H_{i}<\Gamma_{i} \text { finite index, } i=1,2\right\} / \sim,
$$

where isomorphisms $\varphi$ and $\psi$ are equivalent, $\varphi \sim \psi$, if they agree on restriction to a common finite index subgroup of their domains. Elements of $\operatorname{Comm}\left(\Gamma_{1}, \Gamma_{2}\right)$ shall be called abstract commensurators from $\Gamma_{1}$ to $\Gamma_{2}$, and when this set is nonempty we shall say that $\Gamma_{1}$ and $\Gamma_{2}$ are abstractly commensurable. Note that when $\Gamma_{1}$ and $\Gamma_{2}$ are the same group this set has the structure of a group (under composition of isomorphisms after passing to appropriate finite index subgroups). We shall write $\operatorname{Comm}(\Gamma)=\operatorname{Comm}(\Gamma, \Gamma)$ and refer to this as the abstract commensurator group of $\Gamma$. Note that there is a natural homomorphism $\operatorname{Aut}(\Gamma) \rightarrow \operatorname{Comm}(\Gamma)$ whose kernel consists of those automorphisms which fix a finite index subgroup $\Gamma$.

Before continuing, we make some general observations concerning the relationships between a 2-dimensional Artin group, its automorphism group and its abstract commensurator group. If $\Delta$ is a 2-dimensional defining graph with at least 3 vertices then $G=G(\Delta)$ has a trivial centre and so is isomorphic to $\operatorname{Inn}(G)$. Moreover, consideration of Lemma $8(\mathrm{i})$ shows that $s$ is the unique $N$ th root of $s^{N}$ for any generator $s \in V(\Delta)$ and any $N \in \mathbb{N}$. It follows that any automorphism of $G$ which restricts to the identity on a finite index subgroup of $G$ is the identity on all of $G$. Thus the natural homomorphism 
$\operatorname{Aut}(G) \rightarrow \operatorname{Comm}(G)$ is injective. Identifying $\operatorname{Aut}(G)$ with its image, we have

$$
G \cong \operatorname{Inn}(G)<\operatorname{Aut}(G)<\operatorname{Comm}(G) .
$$

We now turn to the class of CLTTF Artin groups. Our principal tool for studying abstract commensurators between these groups is the following structure:

Definition (The fixed set graph $\Theta$ ) Let $\Delta$ denote a CLTTF defining graph. We define the following sets of subsets of the Deligne complex $\mathbb{D}$ of type $\Delta$ :

$$
\begin{aligned}
& \mathcal{V}=\left\{\text { singletons }\left\{g V_{e}\right\}: g \in G, e \in E(\Delta)\right\}, \text { and } \\
& \mathcal{F}=\left\{\text { unbounded trees } g F_{s}: g \in G, s \in V(\Delta)\right\} .
\end{aligned}
$$

We define the fixed set graph $\Theta=\Theta(\Delta)$ to be the bipartite graph with the following vertex and edge sets:

$$
\begin{aligned}
\operatorname{Vert}(\Theta) & :=\mathcal{V} \cup \mathcal{F} \\
\operatorname{Edge}(\Theta) & :=\{(V, F): V \in \mathcal{V}, F \in \mathcal{F} \text { and } V \subset F\} .
\end{aligned}
$$

Observe that, by Lemma 8, Remark [10, and Lemma 11, and since we are supposing large type (LT), we have that

$$
\mathcal{V} \cup \mathcal{F}=\{\operatorname{Fix}(C): C \text { is a CNVA subgroup of } G\},
$$

where $\operatorname{Fix}(C) \in \mathcal{V}$ if $C$ is conjugate to a subgroup of $\left\langle z_{e}\right\rangle$ for some $e \in E(\Delta)$, and $\operatorname{Fix}(C) \in \mathcal{F}$ if $C$ is conjugate to a subgroup of $\langle s\rangle$ for some internal vertex $s \in V(\Delta)$.

Lemma 12 Let $C, C^{\prime}$ be $C N V A$ subgroups of $G$. Then

(i) $C \cap C^{\prime} \neq\{1\}$ if and only if $\operatorname{Fix}(C)=\operatorname{Fix}\left(C^{\prime}\right)$.

(ii) $\left\langle C, C^{\prime}\right\rangle \cong \mathbb{Z} \times \mathbb{Z}$ if and only if $\left(\operatorname{Fix}(C), \operatorname{Fix}\left(C^{\prime}\right)\right) \in \operatorname{Edge}(\Theta)$.

Proof (i) If $\operatorname{Fix}(C)=g F_{s}$ for some $g \in G$ and $s \in V(\Delta)$, then $C<g\langle s\rangle g^{-1}$ since $g V_{s} \in g F_{s}$. On the other hand, if $\operatorname{Fix}(C)=\left\{g V_{e}\right\}$, for some $g \in G$ and $e \in E(\Delta)$, then $C_{G}(C)<g G(e) g^{-1}$ and, by Lemma 7(i), $C<g\left\langle z_{e}\right\rangle g^{-1}$ (else it fails to be CNVA). Therefore, if $\operatorname{Fix}(C)=\operatorname{Fix}\left(C^{\prime}\right)$ then $C$ and $C^{\prime}$ lie in a common infinite cyclic subgroup, so must intersect nontrivially.

On the other hand, it follows from Lemma 8 that a cyclic subgroup of $G$ has the same fixed set as any of its nontrivial subgroups. Thus, if $C^{\prime \prime}=C \cap C^{\prime}$ is nontrivial we have $\operatorname{Fix}(C)=\operatorname{Fix}\left(C^{\prime \prime}\right)=\operatorname{Fix}\left(C^{\prime}\right)$.

(ii) Suppose that $\operatorname{Fix}(C)=V \in \mathcal{V}$ and $\operatorname{Fix}\left(C^{\prime}\right)=F \in \mathcal{F}$ such that $V \subset F$. Up to conjugation of $C, C^{\prime}$ in $G$ we may suppose that $F$ contains the edge 
$\left[V_{e}, V_{s}\right]$, for some $e=\{s, t\} \in E(\Delta)$, and that $V=\left\{V_{e}\right\}$. (This is because any edge of $F$ emanating from $V$ can be viewed as the translate of some edge in the fundamental region $K)$. But then we have $C<\left\langle z_{e}\right\rangle$ (since $\left.\operatorname{Fix}(C)=V_{e}\right)$ and $C^{\prime}<\operatorname{Stab}\left(V_{s}\right)=\langle s\rangle$. Since $\left\langle s, z_{e}\right\rangle \cong \mathbb{Z} \times \mathbb{Z}$ it follows that $\left\langle C, C^{\prime}\right\rangle \cong \mathbb{Z} \times \mathbb{Z}$.

Suppose now that $\left\langle C, C^{\prime}\right\rangle \cong \mathbb{Z} \times \mathbb{Z}$. It follows, since they commute, that $C$ and $C^{\prime}$ have a common fixed point in $\mathbb{D}$, for $C$ must leave $\operatorname{Fix}\left(C^{\prime}\right)$ invariant and so fixes the orthogonal projection $p^{\prime} \in \operatorname{Fix}\left(C^{\prime}\right)$ of any point $p \in \operatorname{Fix}(C)$. However, a rank 2 abelian subgroup can only fix a rank 2 vertex. So $\operatorname{Fix}(C) \cap \operatorname{Fix}\left(C^{\prime}\right)$ consists of a single vertex $V \in \mathcal{V}$, say. Up to conjugation by an element of $G$ we may suppose that $C, C^{\prime}<\operatorname{Stab}\left(V_{e}\right)=G(e)$, for some $e=\{s, t\} \in E(\Delta)$. Each of the two CNVA subgroups is then either a subgroup of $Z(G(e))=\left\langle z_{e}\right\rangle$ or conjugate in $G(e)$ to a subgroup of $\langle s\rangle$ or of $\langle t\rangle$. By Lemma 7(iii) they cannot both be of the latter kind unless they lie in a common cyclic subgroup. Similarly, they cannot both lie in the centre. But then one is central and one is conjugate into $\langle s\rangle$ say. That is to say that, up to conjugacy in $G$, we have $\left\{\operatorname{Fix}(C), \operatorname{Fix}\left(C^{\prime}\right)\right\}=\left\{V_{e}, F_{s}\right\}$.

Proposition 13 Let $\Delta, \Delta^{\prime}$ denote CLTTF defining graphs, and suppose that $\varphi: H \rightarrow H^{\prime}$ is an abstract commensurator from $G(\Delta)$ to $G\left(\Delta^{\prime}\right)$. Then $\varphi$ determines a unique well-defined graph isomorphism $\Phi: \Theta(\Delta) \rightarrow \Theta\left(\Delta^{\prime}\right)$ such that $\Phi(\operatorname{Fix}(C))=\operatorname{Fix}(\varphi(C \cap H))$ for any $C N V A$ subgroup $C$ of $G(\Delta)$.

Proof This is a consequence of Lemma 12 above and the fact that the properties " $C$ is CNVA", " $C \cap C^{\prime} \neq\{1\}$ " and " $\left\langle C, C^{\prime}\right\rangle \cong \mathbb{Z} \times \mathbb{Z}$ " are all preserved by isomorphism and passage to finite index subgroups.

Remark Consider a fixed $\Delta$ of type CLTTF, and write $G=G(\Delta)$ and $\Theta=$ $\Theta(\Delta)$. Note that the action of $G$ on $\mathbb{D}$ induces an action of $G$ by graph automorphisms of $\Theta$. We remark that the action of $\operatorname{Comm}(G)$ on $\Theta$ given by the above Proposition extends this action of $G$ when $G$ is identified with the subgroup of $\operatorname{Comm}(G)$ consisting of inner automorphisms.

\section{Circuits in the graph $\Theta$}

In this and subsequent sections we analyse the structure of the graph of fixed sets associated to a CLTTF defining graph $\Delta$. For simplicity we shall write $\Theta=\Theta(\Delta)$ and $G=G(\Delta)$. 
Lemma 14 If $\Delta_{2}$ denotes the first subdivision of $\Delta$ (so $\Delta_{2}$ has vertex set $E(\Delta) \cup V(\Delta)$ ) we let $\widehat{\Delta}$ denote the full subgraph of $\Delta_{2}$ spanned by the nonterminal vertices. Then there is a graph embedding $f: \widehat{\Delta} \hookrightarrow \Theta$ defined by $f(s)=F_{s}$, if $s \in V(\Delta)$ is an internal vertex, and $f(e)=V_{e}$, if $e \in E(\Delta)$.

Proof It is clear that, as written, $f$ is a well-defined graph morphism. Clearly, also, $f$ is injective on $E(\Delta)$. Suppose $s, t \in V(\Delta)$ and $s \neq t$. Suppose that $F_{s}=F_{t}$. Then, by convexity, $F_{s}$ must contain the geodesic segment $\left[V_{s}, V_{t}\right]$. However, $\left[V_{s}, V_{t}\right]$ intersects the interior of the fundamental region of $\mathbb{D}$, while $F_{s}$ does not, a contradiction. Therefore $f$ is injective on $V(\Delta)$.

From now on we shall identify $\widehat{\Delta}$ with its image $f(\widehat{\Delta})$ in $\Theta$. We also observe that $\Theta$ is the union of translates of the subgraph $\widehat{\Delta}$ by elements of $G$. In particular, since we suppose that $\Delta$ connected (C), we deduce that $\Theta$ is also connected ( $G$ is generated by elements which individually fix some part of $\widehat{\Delta}$ ). A particular consequence of this is that any automorphism of $\Theta$ respects the given bi-partite structure. However, we have not ruled out the possibility that $\Phi(\mathcal{V})=\mathcal{F}$ and $\Phi(\mathcal{F})=\mathcal{V}$ for some $\Phi \in \operatorname{Aut}(\Theta)$.

In order to understand which structural properties of the graph $\Theta$ are respected by graph isomorphisms (coming from abstract commensurators of $G$ ) we shall study the properties of simple closed circuits in $\Theta$.

Let $\Sigma=\left(V_{1}, F_{1}, V_{2}, F_{2}, \ldots, V_{k}, F_{k}\right)$ denote a simple circuit of length $2 k$ in $\Theta$, where $V_{i} \in \mathcal{V}, F_{i} \in \mathcal{F}$, and $V_{i}, V_{i+1} \subset F_{i}$ for each $i=1,2, . ., k$, with indices taken $\bmod k$ (so that $V_{1} \subset F_{k}$ ). Note that $F_{i-1} \cap F_{i}=V_{i}$ and consists of a single rank 2 vertex of $\mathbb{D}$. For each $i=1, . ., k$, let $\gamma_{i}$ denote the geodesic segment in $F_{i}$ from $V_{i}$ to $V_{i+1}$. To the simple circuit $\Sigma$ we associate the closed polygonal curve $\bar{\Sigma}=\left(\gamma_{1}, \ldots, \gamma_{k}\right)$ in $\mathbb{D}$. Note that each segment $\gamma_{i}$ is an edge path in the 1 -skeleton of $\mathbb{D}$ and is geodesic in $\mathbb{D}$ (by convexity of $F_{i}$ ).

Definition (Basic circuit) A simple circuit $\Sigma$ in $\Theta$, and its associated polygon $\bar{\Sigma}$ in $\mathbb{D}$, are said to be basic if $\Sigma$ is the translate by an element of $G$ of a simple circuit in the subgraph $\widehat{\Delta}$, equivalently if the polygon $\bar{\Sigma}$ lies wholly in (the boundary of) a single translate of the fundamental region $K$ in $\mathbb{D}$.

Note that the property of being a basic circuit depends upon the structure of the Deligne complex (rather than just the structure of $\Theta$ ). We wish to characterize certain basic circuits purely in terms of the graph theoretic properties of $\Theta$. 
Definition (Minimal circuit) Let $\Sigma$ denote a simple circuit in $\Theta$. A shortcircuit of $\Sigma$ is any simple path $A$ in $\Theta$ which intersects $\Sigma$ only in its endpoints $P, Q$, and which is strictly shorter than any path in $\Sigma$ from $P$ to $Q$. We say that $\Sigma$ is a minimal circuit if it is a simple circuit and admits no short-circuit. (More succinctly, a circuit is minimal if and only if it is isometrically embedded when $\Theta$ is viewed as a metric graph with edges of constant length). Note that if the circuit $\Sigma$ admits a short circuit $A$ then we may decompose $\Sigma$ into a pair of simple circuits each of length strictly smaller than $\Sigma$, namely:

$$
\Sigma_{1}=A_{1} A \quad \text { and } \quad \Sigma_{2}=A^{-1} A_{2}, \quad \text { where } \quad \Sigma=A_{1} A_{2} .
$$

This provides an inductive procedure for reducing an arbitrary simple circuit into (a finite collection of) minimal circuits.

We devote the next section to proving the following two Propositions.

Proposition 15 Any minimal circuit of $\Theta$ is a basic circuit.

Proposition 16 Any minimal circuit of $\widehat{\Delta}$ is minimal as a circuit of $\Theta$.

Remark While the family of all minimal circuits of the graph $\Theta$ is easily seen to be preserved by any abstract commensurator of $G$, the above Propositions show that this structure in the graph $\Theta$ is also closely related to the combinatorial definition of $G$, and hence to the structure of the Deligne complex $\mathbb{D}$. Namely, the minimal circuits are precisely the translates in $\Theta$ of the minimal circuits of $\widehat{\Delta}$. This connection to the Deligne complex shall be developed further in subsequent sections, and will ultimately lead to the proof of Theorem 3 .

\section{On minimal circuits - Propositions 15 and 16}

Let $\Delta$ be a CLTTF defining graph. Throughout this section we shall regard the associated Deligne complex $\mathbb{D}$ as a squared complex equipped with the cubical metric $d_{C}$. Since $\Delta$ is of type CLTTF, the metric space $\left(\mathbb{D}, d_{C}\right)$ is a $\operatorname{CAT}(0)$ squared complex.

We begin with a useful lemma which reflects the $\delta$-hyperbolicity of the Deligne complex. 
Lemma 17 Let $F \in \mathcal{F}$, and let $\gamma$ be any geodesic segment in $F$ which passes through a rank 2 vertex $p$. Then $\gamma$ is "super-geodesic" at $p$, by which we mean that $\gamma$ enters and leaves $p$ through points separated in $L k(p, \mathbb{D})$ by a path distance strictly greater than $\pi$, in fact at least $3 \pi / 2$.

Proof Let $E, E^{\prime}$ denote the edges of $F$ along which $\gamma$ enters and leaves the point $p$. If $E$ and $E^{\prime}$ define points in $\operatorname{Lk}(p, \mathbb{D})$ which are joined by a path of length $\pi$ then there exist squares $Q, Q^{\prime}$ adjacent to $E, E^{\prime}$ respectively, which share a common edge $E^{\prime \prime}$ such that $E^{\prime \prime} \cap F=\{p\}$. Let $g$ denote a nontrivial element of $\operatorname{Stab}(F)$, and observe that $g\left(E^{\prime \prime}\right) \neq E^{\prime \prime}$. It follows that the squares $Q, Q^{\prime}, g(Q)$ and $g\left(Q^{\prime}\right)$ form a larger square with the vertex $p$ at its centre. In particular we see that $\operatorname{Lk}(p, \mathbb{D})$ contains a simple circuit of length exactly $2 \pi$. Since $G(\Delta)$ is assumed to be large type (LT), the shortest simple circuit in the link of a rank 2 vertex of $\mathbb{D}$ has length at least $3 \pi$ (see [9], also Lemma 39] of Section 10 below), a contradiction. Thus, any path in $L k(p, \mathbb{D})$ from $E$ to $E^{\prime}$ has length strictly greater than $\pi$, and therefore at least $3 \pi / 2$ since all edges of the link graph are of length $\pi / 2$.

In the following arguments we shall use the properties of walls (or hyperplanes) in a CAT(0) cubed complex. The notion appears frequently in the literature. See for example 21, or [19. Two edges in a CAT(0) squared complex $X$ may be said to be parallel if they are opposite edges of the same square in the complex (more generally, if they are parallel edges of the same $n$-cube in the case of a higher dimensional cube complex). This generates an equivalence relation on the set of all edges. By a wall in $X$ we shall mean the convex subspace spanned by the midpoints of the edges lying in a single parallelism class. Since $X$ is $\mathrm{CAT}(0)$ and 2-dimensional this defines a tree which is isometrically embedded in $X$. Moreover, a wall in $X$ separates $X$ into exactly two components, usually called half-spaces.

Definition $\left(\mathbb{F} \subset \mathbb{D} ; W^{+}, W^{-}\right.$and $\partial W^{+}$for a wall $W$ ) It will be convenient to write $\mathbb{F}$ for the subcompex of $\mathbb{D}$ which is the union of the sets $F \in \mathcal{F}$. This is the largest subcomplex of $\mathbb{D}$ which contains no rank 0 vertices, equivalently the set of all points in $\mathbb{D}$ with nontrivial stabilizer in $G(\Delta)$. We note that any wall $W$ in $\mathbb{D}$ may be naturally oriented: we thus denote the connected components of $\mathbb{D} \backslash W$ by $W^{+}, W^{-}$in such a way that every edge of $\mathbb{F}$ which crosses $W$ has a rank 1 vertex in $W^{-}$and a rank 2 vertex in $W^{+}$. (All other edges of $\mathbb{D}$ which cross $W$ have a rank 0 vertex in $W^{-}$and a rank 1 vertex in $W^{+}$). Let $\partial W^{+}$denote the subcomplex of $\mathbb{D}$ spanned by those vertices in $W^{+}$which belong to edges crossing $W$. Thus $\partial W^{+}$is a parallel copy of $W$ spanned by 
vertices of rank 1 and 2 . In particular, $\partial W^{+}$is a convex subtree of $\mathbb{D}$, and also a subcomplex of $\mathbb{F}$.

Definition (Orthogonality) We shall say that two convex subsets of $\mathbb{D}$ intersect orthogonally at a point $p$ if the orthogonal projection of either one to the other contains only the point $p$. Note that convex subsets of the 1-skeleton of the squared complex $\mathbb{D}$ which intersect in a single point always intersect orthogonally, since all angles are multiples of $\frac{\pi}{2}$.

Definition $(\mathcal{V}$-paths $)$ By a $\mathcal{V}$-path we shall mean an edge path in $\Theta$ whose initial and terminal vertices lie in $\mathcal{V}$. Given a $\mathcal{V}$-path $A$ we shall write $\bar{A}$ to denote the piecewise geodesic path in $\mathbb{D}$ induced by $A$ (in the manner described previously for simple circuits). Thus, if $A=\left(V_{1}, F_{1}, V_{2}, F_{2}, \ldots, V_{k}, F_{k}, V_{k+1}\right)$ denotes a $\mathcal{V}$-path of length $2 k$ where $V_{i} \in \mathcal{V}, F_{i} \in \mathcal{F}$ and $V_{i}, V_{i+1} \subset F_{i}$ for $i=1, . ., k$, then $\bar{A}$ is simply the union of the geodesic segments joining $V_{i}$ to $V_{i+1}$ in $F_{i}$, for $i=1, . ., k$.

Given a $\mathcal{V}$-path $A$ in $\Theta$ we shall write $\mathcal{W}(A)$ to denote the set of walls of the squared complex $\mathbb{D}$ which are traversed by the induced path $\bar{A}$. In particular, if $\bar{A}$ happens to be geodesic in $\mathbb{D}$ then $\mathcal{W}(A)$ is exactly the set of walls which separate the endpoints of $\bar{A}$.

We shall use $L(A)$ to denote the edge length of a path $A$ in $\Theta$. For $\mathcal{V}$-paths this length is always even.

Lemma 18 Let $A, B$ denote $\mathcal{V}$-paths in $\Theta$, and write $\alpha=\bar{A}$ and $\beta=\bar{B}$. Suppose that $\alpha$ is a nontrivial geodesic in $\mathbb{D}$ and $\mathcal{W}(A) \subset \mathcal{W}(B)$. Then

(i) $L(A) \leq L(B)$, and

(ii) if, moreover, $\alpha$ and $\beta$ share a common endpoint, $p$, but do not both leave the vertex $p$ along the same edge of $\mathbb{D}$, then the inequality is strict: $L(A)<L(B)$.

Proof (i) We shall compare the number of vertices of type $\mathcal{V}$ appearing along each path. Note that, since $\alpha$ is geodesic and intersects orthogonally with each element of $\mathcal{W}(A)$, the walls $\mathcal{W}(A)$ are mutually disjoint. Let $V$ denote a type $\mathcal{V}$ vertex lying along the path $A$ and let $W_{1}, W_{2}$ denote the walls of $\mathbb{D}$ traversed by $\alpha$ immediately before and after passing through $V$. Since $V$ is a rank 2 vertex, we have $W_{1} \subset W_{2}^{+}$and $W_{2} \subset W_{1}^{+}$. Since it traverses both walls, the path $\beta$ must pass across the region $W_{1}^{+} \cap W_{2}^{+}$between the two walls. We now claim that $B$ also has a vertex of type $\mathcal{V}$ lying in the region $W_{1}^{+} \cap W_{2}^{+}$ 
containing $V$. Since these regions for the different type $\mathcal{V}$ vertices of $A$ are disjoint, it follows that $B$ has at least as many type $\mathcal{V}$ vertices as $A$ does, and hence that $L(A) \leq L(B)$.

To see the claim, observe that, if the path $B$ has no type $\mathcal{V}$ vertex falling between $W_{1}$ and $W_{2}$ then it must contain a subpath $\left(V, F, V^{\prime}\right)$, with $V, V^{\prime} \in$ $\mathcal{V}$ and $F \in \mathcal{F}$, such that the geodesic segment of $\beta$ joining $V$ to $V^{\prime}$ in $F$ crosses both $W_{1}$ and $W_{2}$. Let $a, b, c, d$ denote the four points of intersection between convex sets $\alpha, W_{1}, W_{2}$, and the geodesic segment of $\beta$ just mentioned. Since all intersections are orthogonal, the Flat Quadrilateral Theorem, II.2.11 of [7, implies that the four points $a, b, c, d$ and the geodesic segments connecting them in $\alpha, \beta, W_{1}$ and $W_{2}$ form the boundary of a flat Euclidean rectangle isometrically embedded in $\mathbb{D}$. We conclude, by Lemma 17 that $\beta$ does not pass through any rank 2 vertex between $W_{1}$ and $W_{2}$, since $\beta$ would have to be super-geodesic at any such vertex. This is of course a contradiction, since any edge of $\mathbb{F}$ which crosses $W_{1}$ in the direction of $W_{2}$ immediately encounters a rank 2 vertex (because $W_{2} \subset W_{1}^{+}$).

(ii) Suppose without loss of generality that $p$ is the initial vertex of both $\alpha$ and $\beta$. Note that, since $\alpha$ is nontrivial, $0 \neq L(A) \leq L(B)$ and we may write $B=\left(V, F, B^{\prime}\right)$ where $V=\{p\}, F \in \mathcal{F}$, and $B^{\prime}$ is a $\mathcal{V}$-path of strictly smaller length: $L\left(B^{\prime}\right)=L(B)-2$. Since $\alpha$ and $\beta$ set off along different edges of the Deligne complex, the convex sets $\beta \cap F$ and $\alpha$ must intersect orthogonally at $p$. It follows that no wall of $\mathcal{W}(A)$ can cross $\beta \cap F$, so $\mathcal{W}(A) \subset \mathcal{W}\left(B^{\prime}\right)$. Applying part (i) of the Lemma, we conclude that $L(A) \leq L\left(B^{\prime}\right)<L(B)$.

Definition (Chords) Let $\Sigma$ denote a simple circuit in $\Theta$, and $\bar{\Sigma}$ the corresponding piecewise geodesic closed curve in $\mathbb{D}$. By a chord of $\bar{\Sigma}$ we mean a path $\alpha$ from $p$ to $q$ such that

(C1) $\alpha$ is a geodesic path in $\mathbb{D}$ and is contained in the subcomplex $\mathbb{F}$,

(C2) $\alpha \cap \bar{\Sigma}=\{p, q\}$ where the endpoints $p$ and $q$ are vertices of $\mathbb{D}$.

Observe that the endpoints $p, q$ of a chord $\alpha$ serve to cut $\bar{\Sigma}$ into a concatenation of two paths $\sigma_{1}$ and $\sigma_{2}$ each joining $p$ to $q$. We shall say that $\alpha$ is aligned with $\sigma_{1}$ if $\alpha$ and $\sigma_{1}$ form a right angle at each endpoint.

A rank 2 vertex lying on the path $\bar{\Sigma}$ shall be termed essential if it corresponds to a type $\mathcal{V}$ vertex of $\Sigma$, and inessential otherwise.

Lemma 19 Let $\Sigma$ denote a minimal circuit of $\Theta$. Then

(i) $\bar{\Sigma}$ is a simple closed curve in $\mathbb{D}$, and 
(ii) if $\alpha$ is a chord of $\bar{\Sigma}$, cutting $\bar{\Sigma}$ into subpaths $\sigma_{1}, \sigma_{2}$, then both endpoints of $\alpha$ are inessential rank 2 vertices and $\alpha$ is aligned with either $\sigma_{1}$ or $\sigma_{2}$ (but not with both).

Proof (i) The essential vertices of $\bar{\Sigma}$ are clearly mutually distinct, since $\Sigma$ is a simple circuit of $\Theta$. Suppose then that $p$ is a point which lies on the interior of more than one "side" of $\bar{\Sigma}$, and let $P, Q$ denote fixed trees which appear as distinct vertices of $\Sigma$ (of type $\mathcal{F}$ ) and which both contain $p$. Then $P \cap Q=V$, where $V=\{p\} \in \mathcal{V}$. In particular, $V$ is the unique element of $\mathcal{V}$ which is adjacent, in $\Theta$, to both $P$ and $Q$. It follows that $(P, V, Q)$ defines a short circuit for $\Sigma$, contradicting the minimality of $\Sigma$.

(ii) Let $P$ (resp. $Q$ ) denote the smallest fixed set which appears as a vertex of $\Sigma$ and which contains the endpoint $p$ (resp. $q$ ) of $\alpha$. Either $P=\{p\} \in \mathcal{V}$, or $P \in \mathcal{F}$ is an unbounded tree containing $p$ (similarly for $Q$ ). For any $\mathcal{V}$-path $X$ in $\Theta$ with endpoints contained in $P$ and $Q$ respectively we shall denote by $P X Q$ the path in $\Theta$ obtained by appending the vertices $P$ and/or $Q$ whenever they belong to $\mathcal{F}$ (ie, whenever they are not already endpoints of $X$ ).

Let $\sigma_{1}^{\prime}, \sigma_{2}^{\prime}$ and $\alpha^{\prime}$ denote the shortest subpaths of $\sigma_{1}, \sigma_{2}$, and $\alpha$ respectively, which have an endpoint in each of the sets $P$ and $Q$. Note that the endpoints of these subpaths are all necessarily rank 2 vertices (since the paths all lie in $\mathbb{F}$, which is the union of the elements of $\mathcal{F}$, and any two elements of $\mathcal{F}$ meet, if at all, in a vertex of rank 2). Now $\Sigma$ may be expressed as the union of paths $P \Sigma_{1} Q$ and $P \Sigma_{2} Q$ where $\Sigma_{1}$ and $\Sigma_{2}$ are $\mathcal{V}$-paths with $\bar{\Sigma}_{i}=\sigma_{i}^{\prime}$, for $i=1,2$. One may also easily construct a $\mathcal{V}$-path $A$, with endpoints in $P$ and $Q$, such that $\bar{A}=\alpha^{\prime}$. We observe that $\alpha^{\prime}$ intersects the sets $P$ and $Q$ orthogonally at its endpoints. This ensures that $\mathcal{W}(A) \subset \mathcal{W}\left(\Sigma_{i}\right)$ for each $i=1,2$.

If either $P \in \mathcal{V}$ or $Q \in \mathcal{V}$ then it follows from Lemma 18(ii) that $L(A)<L\left(\Sigma_{i}\right)$ for $i=1,2$. But then $P A Q$ is a short circuit for $\Sigma$, contradicting minimality.

Now suppose that both $P, Q \in \mathcal{F}$. In particular, each of the endpoints $p, q$ of $\alpha$ is either rank 1 or an inessential rank 2 vertex of $\bar{\Sigma}$. Note that if $p$ is a rank 1 vertex then $\sigma_{1} \cup \alpha$ is geodesic at the point $p$. This is also the case if $p$ is rank 2, as long as $\alpha$ and $\sigma_{1}$ do not form a right angle at $p$. In either case $\alpha^{\prime}$ extends (through $P$ ) to a geodesic having an endpoint, $v$ say, in common with $\sigma_{1}^{\prime}$. Applying Lemma 18 to the $\mathcal{V}$-paths $(\{v\}, P, A)$ and $\Sigma_{1}$ now shows that $L(A)<L\left(\Sigma_{1}\right)$, except possibly when $p$ is inessential, rank 2 and $\alpha$ forms a right angle at $p$ with $\sigma_{1}$. By applying this argument at each end of $\alpha$ and with respect to both $\Sigma_{1}$ and $\Sigma_{2}$, we conclude that $P A Q$ defines a short-circuit 
for $\Sigma$ unless both $p$ and $q$ are inessential rank 2 vertices of $\bar{\Sigma}$ and $\alpha$ is aligned with one of $\sigma_{1}$ or $\sigma_{2}$. Finally, note that $\alpha$ cannot be aligned with both $\sigma_{1}$ and $\sigma_{2}$ since $\bar{\Sigma}$ is super-geodesic at $p$ and $q$, by Lemma 17.

Lemma 20 Let $\Sigma$ be a minimal circuit of $\Theta$, and suppose that all rank 2 vertices of $\bar{\Sigma}$ are essential. Then $\Sigma$ is a basic circuit.

Proof The fact that all rank 2 vertices are essential implies, by Lemma 19](ii), that $\bar{\Sigma}$ has no chords. It follows that, for every wall $W$ which intersects $\bar{\Sigma}$, the set $\partial W^{+} \cap \bar{\Sigma}$ is connected. For, otherwise, any shortest length path in $\partial W^{+}$ joining distinct components of $\partial W^{+} \cap \bar{\Sigma}$ would be a chord of $\bar{\Sigma}$.

Given a wall $W$, we shall define $N\left(W^{-}\right)$to be the convex hull in $\mathbb{D}$ of the subsets $W^{-}$and $\partial W^{+}$. It follows from the connectivity statement above and the fact that $\bar{\Sigma}$ is a simple closed curve (Lemma 19(i)) that, for any wall $W$ of $\mathbb{D}$, either $\bar{\Sigma} \subset W^{+}$or $\bar{\Sigma} \subset N\left(W^{-}\right)$.

Consider the fundamental region $K$ of $\mathbb{D}$, with rank 0 vertex $V_{\emptyset}$. For each $t \in V(\Delta)$ we define $W_{t}$ to be the unique wall of $\mathbb{D}$ which cuts the edge $\left[V_{\emptyset}, V_{t}\right]$. These are exactly the walls of $\mathbb{D}$ which intersect nontrivially with $K$. Moreover, we have

$$
K=\bigcap_{t \in V(\Delta)} N\left(W_{t}^{-}\right)
$$

Let $W_{0}$ be any wall crossed by $\bar{\Sigma}$. Note that, since $\bar{\Sigma}$ is a simple closed curve it must cross $W_{0}$ in at least two different places. The intersection $\bar{\Sigma} \cap \partial W_{0}^{+}$must therefore contain at least two rank 2 vertices and, by connectedness, at least one rank 1 vertex. Let $v$ denote such a rank 1 vertex in $\bar{\Sigma} \cap \partial W_{0}^{+}$, and let $u$ be the unique rank 0 vertex such that $u$ is adjacent to $v$ and the edge $[u, v]$ crosses $W_{0}$. Up to an isometry of $\mathbb{D}$ we may as well suppose that $[u, v]=\left[V_{\emptyset}, V_{s}\right] \subset K$ for some $s \in V(\Delta)$, and hence that $W_{0}=W_{s}$. Note that $W_{s}$ is the unique wall which separates $V_{s}$ from $V_{\emptyset}$. It follows that, for any $t \in V(\Delta)$ different from $s$, we have $V_{s} \subset W_{t}^{-}$. Thus $\bar{\Sigma} \subset N\left(W_{t}^{-}\right)$(as $\bar{\Sigma}$ is clearly not contained in $W_{t}^{+}$).

Since $\bar{\Sigma}$ crosses the wall $W_{s}$, we must also have $\bar{\Sigma} \subset N\left(W_{s}^{-}\right)$. It follows that $\bar{\Sigma} \subset K$, and so $\Sigma$ is a basic circuit.

\subsection{Proof of Proposition 15}

Let $\Sigma$ denote a minimal circuit in $\Theta$. In order to show that $\Sigma$ is basic it will suffice, by Lemma 20. to show that every rank 2 vertex of $\bar{\Sigma}$ is essential (ie, corresponds to a vertex of $\Sigma$ of type $\mathcal{V}$ ). 
Suppose that $p$ is an inessential rank 2 vertex on $\bar{\Sigma}$. Let $E_{1}, E_{2}$ denote the two edges of $\bar{\Sigma}$ adjacent to $p$, and $W_{1}, W_{2}$ the corresponding walls ( $W_{i}$ is the unique wall of $\mathbb{D}$ which cuts across the edge $E_{i}$ ). Since $\bar{\Sigma}$ is geodesic at $p$ (in fact super-geodesic) and also a simple closed curve (by Lemma 19(i)), the point $p$ is an isolated point of the set $\bar{\Sigma} \cap \partial W_{1}^{+}$. Also, since $W_{1}$ is separating and $\bar{\Sigma}$ is a simple closed curve, the set $\bar{\Sigma} \cap \partial W_{1}^{+}$must contain points other than $p$. Any shortest length path in $\partial W_{1}^{+}$joining $p$ to another component of $\partial W_{1}^{+} \cap \bar{\Sigma}$ now defines a chord of $\bar{\Sigma}$, which we shall denote $\alpha_{1}$. Note that $\alpha_{1}$ forms a right angle with $\sigma_{1}$ at $p$. Similarly, there exists a second chord $\alpha_{2}$ which lies in $\partial W_{2}^{+}$ and and forms a right angle at $p$ with $\sigma_{2}$. Let $q_{1}, q_{2}$ denote the endpoints of $\alpha_{1}, \alpha_{2}$ respectively, which are different from $p$, and write $\bar{\Sigma}$ as a union of paths $\sigma_{1}$ from $p$ to $q_{1}, \sigma_{2}$ from $p$ to $q_{2}$, and $\sigma_{3}$ from $q_{1}$ to $q_{2}$. By Lemma 19](ii), $q_{1}$ and $q_{2}$ are both inessential rank 2 vertices, and $\alpha_{i}$ is necessarily aligned with $\sigma_{i}$, for each $i=1,2$. The situation is illustrated in Figure 2, We shall show that the union of the chords $\alpha_{1}$ and $\alpha_{2}$ represents a short-circuit of $\Sigma$, and thereby obtain a contradiction.

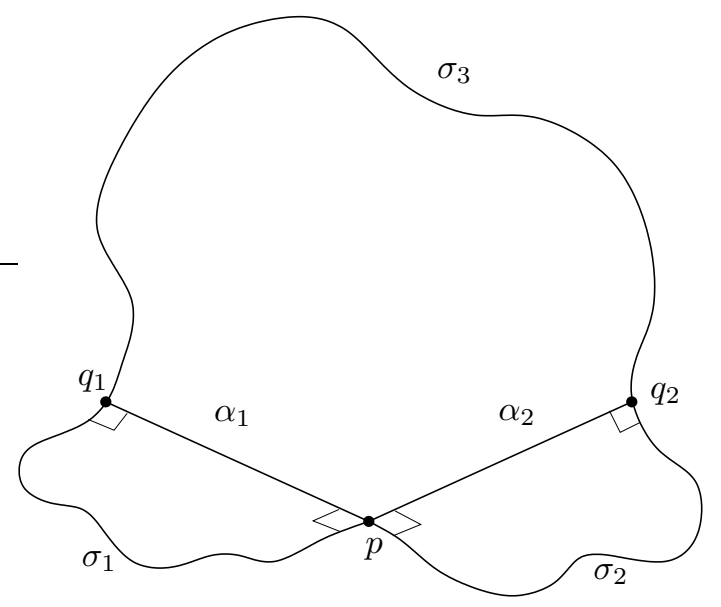

Figure 2: Chords $\alpha_{i}$ aligned with $\sigma_{i}$ for $i=1,2$

Write $P, Q_{1}$ and $Q_{2}$ for the vertices of type $\mathcal{F}$ in $\Sigma$ which correspond to fixed sets containing the points $p, q_{1}$ and $q_{2}$ respectively. By Lemma [17 $\bar{\Sigma}$ is super-geodesic at $p, q_{1}$ and $q_{2}$. It follows that the path $\alpha_{1} \cup \sigma_{3} \cup \alpha_{2}$ is geodesic at both points $q_{1}$ and $q_{2}$, and also that $\alpha_{1} \cap \alpha_{2}=\{p\}$, or rather that $\alpha_{1}$ and $\alpha_{2}$ intersect orthogonally at $p$.

For $i=1,2$, let $A_{i}$ denote the $\mathcal{V}$-path such that $\bar{A}_{i}=\alpha_{i}$. We may suppose 
that, for each $i=1,2$, the path $A_{i}$ is written

$$
A_{i}=\left(V_{1}^{(i)}, F_{1}^{(i)}, V_{2}^{(i)}, F_{2}^{(i)}, \ldots, V_{m_{i}}^{(i)}\right), m_{i} \in \mathbb{N}
$$

where $V_{1}^{(1)}=V_{1}^{(2)}=\{p\} \subset P$ and $V_{m_{i}}^{(i)}=\left\{q_{i}\right\} \subset Q_{i}$, for $i=1,2$.

Let $\Sigma_{3}$ denote the longest $\mathcal{V}$-subpath of $\Sigma$ such that $\bar{\Sigma}_{3}$ is a subpath of $\sigma_{3}$, and write $U_{1} \subset Q_{1}$ and $U_{2} \subset Q_{2}$ for the endpoints of $\Sigma_{3}$.

We now define $\mathcal{V}$-paths $A_{i}^{\prime}$ for $i=1,2$ thus:

$$
A_{i}^{\prime}=\left(V_{2}^{(i)}, F_{2}^{(i)}, \ldots, V_{m_{i}}^{(i)}, Q_{i}, U_{i}\right) .
$$

By the preceding remark on geodicity at the points $q_{i}$, the path $\bar{A}_{i}^{\prime}$ is a geodesic in $\mathbb{D}$ and $L\left(A_{i}^{\prime}\right)=L\left(A_{i}\right)$, for each $i=1,2$.

We now make the following claim:

Claim The first walls crossed by $\bar{A}_{1}^{\prime}$ and $\bar{A}_{2}^{\prime}$ are disjoint and separate $U_{1}$ from $U_{2}$.

Proof To see this, let $\gamma_{i}$, for $i=1,2$, denote the geodesic from $\{p\}$ to $U_{i}$ obtained by combining $\overline{A_{i}}$ and $\overline{A_{i}^{\prime}}$. Either the first walls crossed by $\gamma_{1}$ and $\gamma_{2}$ have the desired properties, and hence also the first walls of $\overline{A_{1}^{\prime}}$ and $\overline{A_{2}^{\prime}}$, or the first walls crossed by $\gamma_{1}$ and $\gamma_{2}$ intersect in a square of $\mathbb{D}$ with rank 0 vertex $v_{0}$ and rank 2 vertex $p$. The vertex $v_{0}$ is the centre of a region $K_{0}=g_{0}(K)$ for some $g_{0} \in G$, and the geodesics $\gamma_{1}, \gamma_{2}$ each intersect $K_{0}$ in a subpath of edge length 2 , and leave $K_{0}$ at the rank 2 vertex $p_{1}$, respectively $p_{2}$. Let $W_{i}$ denote the third wall crossed by $\gamma_{i}$ (for $i=1,2$ ), namely the first wall immediately after the vertex $p_{i}$. Then $W_{i}$ is either equal to or precedes the first wall crossed by $\overline{A_{i}^{\prime}}$. Now, if $W_{1} \cap W_{2} \neq \emptyset$ then $\gamma_{1}, \gamma_{2}, W_{1}$, and $W_{2}$ must together bound a flat rectangle isometrically embedded in $\mathbb{D}$ (just as in the proof of Lemma 177). Moreover, this rectangle must contain the vertex $v_{0}$ and at least one rank 2 vertex of $K_{0}$ in its interior. This contradicts the fact that the shortest closed circuit in the link of any rank 2 vertex of $\mathbb{D}$ has length at least $3 \pi$ (cf, the proof of Lemma 17 and Lemma 39 to follow). Thus $W_{1}$ and $W_{2}$ are disjoint with $K_{0}$ lying between them. Moreover $W_{i}$ separates $U_{i}$ from $K_{0}$, for each $i=1,2$. So each $W_{i}$ separates $U_{1}$ from $U_{2}$.

It follows from the claim that $\mathcal{W}\left(A_{1}^{\prime}\right) \cup \mathcal{W}\left(A_{2}^{\prime}\right)$ is a set of mutually disjoint walls all of which cross $\bar{\Sigma}_{3}$. Moreover, we can describe $\Sigma_{3}$ as a union of two $\mathcal{V}$-paths $B_{1}$ and $B_{2}$, where $\mathcal{W}\left(A_{i}^{\prime}\right) \subset \mathcal{W}\left(B_{i}\right)$ for each $i=1,2$, and such that $B_{1}$ and $B_{2}$ overlap in at most 2 edges (ie, at most 2 type $\mathcal{V}$ vertices and one type $\mathcal{F}$ 
vertex in common). Applying Lemma 18(ii), we have $L\left(A_{i}^{\prime}\right) \leq L\left(B_{i}\right)-2$, for each $i=1,2$, and therefore

$$
L\left(A_{1} \cup A_{2}\right)=L\left(A_{1}^{\prime}\right)+L\left(A_{2}^{\prime}\right) \leq L\left(B_{1}\right)+L\left(B_{2}\right)-4 \leq L\left(\Sigma_{3}\right)-2 .
$$

Note that $\Sigma$ may be written $\left(P, \Sigma_{1}, Q_{1}, \Sigma_{3}, Q_{2}, \Sigma_{2}, P\right)$, where $\Sigma_{i}$ denotes the longest $\mathcal{V}$-subpath of $\Sigma$ such that $\bar{\Sigma}_{i}$ is a subpath of $\sigma_{i}$. The above argument shows that $L\left(A_{1} \cup A_{2}\right)<L\left(\Sigma_{3}\right)$. On the other hand, by Lemma 18(i), we have $L\left(A_{i}\right) \leq L\left(\Sigma_{i}\right)$ for $i=1,2$, and therefore, $L\left(A_{1} \cup A_{2}\right)<L\left(\Sigma_{2}, P, \Sigma_{1}\right)$. It follows that $\left(Q_{1}, A_{1}, A_{2}, Q_{2}\right)$ is a short circuit for $\Sigma$. This contradicts the hypothesis that $\Sigma$ is minimal, and completes the proof of Proposition 15 .

\subsection{Proof of Proposition 16}

Recall that, for $s \in V(\Delta)$, we denote by $W_{s}$ the wall in $\mathbb{D}$ perpendicular to the edge $\left[V_{\emptyset}, V_{s}\right]$, and by $W_{s}^{+}$the half-space bounded by $W_{s}$ and containing the vertex $V_{s}$. We observe that any rank 2 vertex $p$ lies in at least one of the half-spaces $W_{s}^{+}$, for $s \in V(\Delta)$, and never more than two. If $e=\{s, t\}$, then $V_{e} \in W_{s}^{+} \cap W_{t}^{+}$. We shall say that a rank 2 vertex projects to the edge $e=\{s, t\}$ if it lies in the region $W_{s}^{+} \cap W_{t}^{+}$. Note that if a rank 2 vertex of some $F_{s}$, for $s \in V(\Delta)$, projects onto an edge then that edge contains $s$, simply because $F_{s} \subset W_{s}^{+}$.

Let $\gamma$ be any path in $\mathbb{F}$ which starts and ends at rank 2 vertices which project to edges, say $e, f \in E(\Delta)$. From the sequence of rank 2 vertices visited by $\gamma$ choose a subsequence $\left(p_{1}, p_{2}, . ., p_{n}\right)$ such that $p_{1}$ projects to $e, p_{n}$ projects to $f$ and, for $i=1, . ., n$, the vertex $p_{i}$ projects to an edge $e_{i}=\left\{s_{i-1}, s_{i}\right\}$, for some sequence $s_{0}, s_{1}, \ldots, s_{n} \in V(\Delta)$. By passing to a subsequence if necessary, we may suppose that the sequence of edges $\left(e_{1}, . ., e_{n}\right)$ describes a simple path in $\Delta$ (from $s_{0}$ to $s_{n}$ ), where $e_{1}=e, e_{n}=f$. This leads to the definition of the following simple $\mathcal{V}$-path in $\widehat{\Delta} \subset \Theta$ :

$$
P(\gamma)=\left(V_{e}=V_{e_{1}}, F_{s_{1}}, V_{e_{2}}, F_{s_{2}}, . ., F_{s_{n-1}}, V_{e_{n}}=V_{f}\right) .
$$

Now suppose that the original path $\gamma$ was given as $\gamma=\bar{A}$ for some $\mathcal{V}$-path $A$ in $\Theta$. Consider an arbitrary edge $\{s, t\} \in E(\Delta)$. If $\gamma$ ever enters the region $W_{s}^{+} \cap W_{t}^{+}$then we claim that $A$ has a type $\mathcal{V}$ vertex which lies in $W_{s}^{+} \cap W_{t}^{+}$. If not, then some type $\mathcal{F}$ vertex of $A$, say $Q \in \mathcal{F}$, must contain a geodesic segment which enters and leaves the region. But this is impossible since $W_{s}$ and $W_{t}$ intersect orthogonally, and $Q$ intersects orthogonally with any wall that it encounters. It follows that, for each $i=1, . ., n$, there is at least one vertex of type $\mathcal{V}$ in $A$ which projects to $e_{i}$. Therefore $L(P(\bar{A}))=L(P(\gamma)) \leq L(A)$. 
Finally we claim that if a basic circuit $\Sigma$ in $\widehat{\Delta}$ admits a short-circuit $B$ in $\Theta$ then it admits a short-circuit $B^{\prime}$ in $\widehat{\Delta}$, for if $A$ denotes the longest subpath of $B$ which is a $\mathcal{V}$-path then replacing $A$ with $P(\bar{A})$ gives the desired path. Proposition 16] now follows.

\section{Minimal circuits, chunks, and isomorphisms of $\Theta$}

Throughout this section we suppose that $\Delta, \Delta^{\prime} \in \mathcal{G}$ (CLTTF defining graphs) and write $\Theta=\Theta(\Delta)$ and $\Theta^{\prime}=\Theta\left(\Delta^{\prime}\right)$. We shall be concerned with graph isomorphisms $\Theta \rightarrow \Theta^{\prime}$ which map $\mathcal{F}$ to $\mathcal{F}^{\prime}$ and $\mathcal{V}$ to $\mathcal{V}^{\prime}$. We call such an isomorphism a $\mathcal{V} \mathcal{F}$-isomorphism of $\Theta$.

Our objective is to describe a decomposition of the graph $\Theta$ which is closely related to the structure of the Deligne complex $\mathbb{D}$ and yet is canonical in the sense that it is respected by any $\mathcal{V} \mathcal{F}$-isomorphism of $\Theta$. The main technical idea is that the pieces of the decomposition (called "chunks") may be characterised by studying the minimal circuits of $\Theta$ introduced in Section 5 .

Definition (Chunks of $\Theta$ ) Let $A$ be a connected full subgraph of $\Delta$. We shall say that $A$ is indecomposable if, for every decomposition, $\Delta=\Delta_{1} \cup_{T} \Delta_{2}$, of $\Delta$ over a separating edge or vertex $T$, either $A \subset \Delta_{1}$ or $A \subset \Delta_{2}$. By a chunk of $\Delta$ we mean a maximal indecomposable (connected and full) subgraph of $\Delta$. Clearly, any two chunks of $\Delta$ intersect, if at all, along a single separating edge or vertex. A chunk of $\Delta$ shall be said to be solid if it contains a simple closed circuit of $\Delta$. It is easy to see that any chunk is either solid or consists of just a single edge of the graph.

Recall that the graph $\widehat{\Delta}$ may be viewed both as a subset of $\Delta$ and as a subgraph of $\Theta$. By a (solid) chunk of $\widehat{\Delta}$ we mean the intersection of $\widehat{\Delta}$ with a (solid) chunk of $\Delta$. This defines a subgraph of $\widehat{\Delta}$ which shall be thought of as lying inside $\Theta$. Finally, we define a (solid) chunk of $\Theta$ to be any translate of a (solid) chunk of $\widehat{\Delta}$ by an element of $G(\Delta)$. A chunk of $\Theta$ is said to be fundamental if it lies in the fundamental subgraph $\widehat{\Delta}$.

Note that a chunk of $\Theta$ is solid if and only if it contains a simple closed circuit of $\Theta$. Any non-solid chunk consists of a path in $\Theta$ of the form $\left(g F_{s}, g V_{e}, g F_{t}\right)$ for $g \in G(\Delta)$ and $e=\{s, t\} \in E(\Delta)$ where $s$ and $t$ are both separating in $\Delta$, or of the form $\left(g F_{s}, g V_{e}\right)$ if $s$ is separating but $t$ is terminal.

Lemma 21 Every minimal circuit of $\Theta$ is contained in a unique solid chunk and each solid chunk of $\Theta$ is the union of the minimal circuits which it contains. 
Proof It is a straightforward exercise to prove the corresponding statements for minimal circuits of $\widehat{\Delta}$ (or $\Delta$ ). The Lemma is then a consequence of Propositions 15 and 16] which establish that the minimal circuits of $\Theta$ are precisely the translates of the minimal circuits of $\widehat{\Delta}$, together with the fact that each basic circuit lies in a unique translate of $\widehat{\Delta}-$ cf Lemma [24(i) below.

Definition 22 (Equivalence of minimal circuits, $\sim$ ) We say that minimal circuits $\Sigma$ and $\Sigma^{\prime}$ of $\Theta$ are equivalent, written $\Sigma \sim \Sigma^{\prime}$, if they are related by a finite sequence of the following type of elementary equivalence:

- $\Sigma \sim \Sigma^{\prime}$ in one step if $\Sigma$ and $\Sigma^{\prime}$ share a common subpath $(S, V, T)$, with $V \in \mathcal{V}$ and $S, T \in \mathcal{F}$, and there exists a sequence of minimal circuits

$$
\Sigma=\Sigma_{0}, \Sigma_{1}, \Sigma_{2}, \ldots, \Sigma_{k}=\Sigma^{\prime}
$$

such that, for $i=1, . ., k$, the circuits $\Sigma_{i}$ and $\Sigma_{i-1}$ share a common subpath $\left(S, V_{i}, T_{i}\right)$ where $V_{i} \neq V$.

It is easily seen that the equivalence relation just defined is necessarily respected by any $\mathcal{V} \mathcal{F}$-isomorphism of $\Theta$. Our interest in this equivalence relation lies in the following Proposition.

Proposition 23 Let $\Theta, \Theta^{\prime}$ denote fixed set graphs of CLTTF type. Suppose that $\Sigma_{1}, \Sigma_{2}$ are minimal circuits in $\Theta$. Then $\Sigma_{1} \sim \Sigma_{2}$ if and only if $\Sigma_{1}$ and $\Sigma_{2}$ belong to the same chunk of $\Theta$. Consequently, any $\mathcal{V} \mathcal{F}$-isomorphism $\Theta \rightarrow \Theta^{\prime}$ maps each solid chunk of $\Theta$ onto a solid chunk of $\Theta^{\prime}$ (inducing a bijection between the solid chunks of $\Theta$ and those of $\Theta^{\prime}$ ).

Remark Note that, by combining Lemma 21] with the above Proposition, any solid chunk of $\Theta$ may be described as just the union of a certain equivalence class of minimal circuits.

The remainder of this section is devoted to the proof of Proposition 23. The Proposition is a consequence of Lemmas 27 and 30 proved in the following two Subsections.

\subsection{Equivalent circuits are in the same chunk}

Recall that if $e=\{s, t\} \in E(\Delta)$ we set $x_{e}$ to be the group element expressed by the word sts.. of length $m_{e}$. Thus $z_{e}=x_{e}$ if $m_{e}$ is even, and $z_{e}=x_{e}^{2}$ if $m_{e}$ is odd. In the latter case, conjugation by $x_{e}$ exchanges the two generators $s$ and 
$t$. The element $x_{e}$ generates the quasi-centre of $G(e)$, the subgroup of elements which respect the set $\{s, t\}$ by conjugation. Note also that, when viewing the action of $G$ on the Deligne complex $\mathbb{D}$, we have $\operatorname{Fix}\left(x_{e}\right)=\operatorname{Fix}\left(z_{e}\right)=V_{e}-\mathrm{cf}$ Lemma 8 (ii).

Definition (Basic solid subset) We define a basic solid subset of $\Theta$ to be any translate, by an element of $G(\Delta)$, of a subgraph of $\widehat{\Delta}$ which contains at least one simple circuit. It follows from Lemma 24(i) below that there is a well-defined function

$$
\beta:\{\text { basic solid subsets of } \Theta\} \rightarrow G(\Delta)
$$

such that $\beta(\Sigma)$ is the unique group element for which $\beta(\Sigma) \widehat{\Delta}$ contains $\Sigma$.

Lemma 24 Let $\Sigma_{1}, \Sigma_{2}$ denote basic solid subsets of $\Theta$, and suppose that these may be written $\Sigma_{1}=\beta_{1} A_{1}$ and $\Sigma_{2}=\beta_{2} A_{2}$ for $\beta_{1}, \beta_{2} \in G(\Delta)$ and $A_{1}, A_{2} \subset \widehat{\Delta}$.

(i) If $\Sigma_{1}$ and $\Sigma_{2}$ share a common path of length $\geq 3$ (so at least a path $\left(V^{\prime}, F, V, F^{\prime}\right)$ with $V, V^{\prime} \in \mathcal{V}$ and $\left.F, F^{\prime}=\mathcal{F}\right)$ then $\beta_{1}=\beta_{2}$.

(ii) If $\Sigma_{1}$ and $\Sigma_{2}$ share a common path $\left(V, F, V^{\prime}\right)$ where $V, V^{\prime} \in \mathcal{V}$ and $F=\beta_{1} F_{s}$ for some $s \in V(\Delta)$, then $\beta_{2}=\beta_{1} h$ where $h \in\langle s\rangle$.

(iii) If $\Sigma_{1}$ and $\Sigma_{2}$ share a common path $\left(F, V, F^{\prime}\right)$ where $F, F^{\prime} \in \mathcal{F}$ and $V=\beta_{1} V_{e}$ for some $e \in E(\Delta)$, then $\beta_{2}=\beta_{1} h$ where $h \in\left\langle x_{e}\right\rangle$.

Proof Note that (i) is a consequence of (ii) and (iii) together with the fact that the cyclic groups $\langle s\rangle$, for $s \in V(\Delta)$, and $\left\langle x_{e}\right\rangle$, for $e \in E(\Delta)$, intersect trivially. (Consider a common path $\left(V^{\prime}, F, V, F^{\prime}\right)$ ).

We assume for simplicity, in each case, that $\beta_{1}=1$. Suppose that, as in case (ii), $\Sigma_{1}$ and $\Sigma_{2}$ share a common path $\left(V_{e}, F_{s}, V_{f}\right)$ where $e, f \in E(\Delta)$ and $s \in V(\Delta)$ with $e \cap f=\{s\}$. Then, since $V_{e}$ and $V_{f}$ lie in distinct $G$-orbits of the action on $\mathbb{D}$, we must have $\beta_{2}\left(V_{e}\right)=V_{e}$ and $\beta_{2}\left(V_{f}\right)=V_{f}$. But then $\beta_{2} \in G(e) \cap G(f)=\langle s\rangle$.

If, as in case (iii), $\Sigma_{1}$ and $\Sigma_{2}$ share a common path $\left(F_{s}, V_{e}, F_{t}\right)$ where $e=$ $\{s, t\} \in E(\Delta)$, then $\beta_{2}\left(V_{e}\right)=V_{e}$ and $\beta_{2}\left(\left\{F_{s}, F_{t}\right\}\right)=\left\{F_{s}, F_{t}\right\}$. Thus $\beta_{2} \in G(e)$ and conjugation by $\beta_{2}$ preserves the set $\{s, t\}$. In other words, $\beta_{2}$ lies in the "quasi-centre" of $G(e)$ which is generated by $x_{e}$.

Lemma 25 Let $G=G(\Delta)$ be a large-type triangle-free Artin group. Then the set $\left\{x_{e}: e \in E(\Delta)\right\}$ freely generates a free subgroup of $G(\Delta)$. 
Proof Let $\mathcal{X}$ denote the complex of groups with underlying complex $K$, a vertex group $\left\langle x_{e}\right\rangle$ at each vertex $V_{e}$, for $e \in E(\Delta)$, and all other vertex groups trivial. Then $\pi_{1}(\mathcal{X})$ is simply the free product of the cyclic groups $\left\langle x_{e}\right\rangle$. (In fact the the universal covering complex $\widetilde{\mathcal{X}}$ admits an equivariant deformation retraction onto a Bass-Serre tree for this free product). The obvious map from $\pi_{1}(\mathcal{X})$ onto the subgroup of $G$ generated by the set $\left\{x_{e}: e \in E(\Delta)\right\}$ is associated with an equivariant map $\psi: \widetilde{\mathcal{X}} \rightarrow \mathbb{D}$ with image the union of translates $g K$ of the fundamental region $K$, for $g \in\left\langle x_{e}: e \in E(\Delta)\right\rangle$. We equip both spaces $\widetilde{\mathcal{X}}$ and $\mathbb{D}$ with the natural cubical metric $d_{C}$, and observe that, for each edge $e \in E(\Delta)$, the union $\bigcup_{k \in \mathbb{Z}} x_{e}^{k} K$ is a convex subset of $\left(\mathbb{D}, d_{C}\right)$ - one simply needs to check local convexity at the vertex $V_{e}$, using the hypothesis that $G$ be large type. It follows that the map $\psi$ is locally isometric, and hence a globally isometric embedding (since the image lies in a CAT(0) space). The map $\pi_{1}(\mathcal{X}) \rightarrow G$ is therefore injective.

Remark Note that the convexity statement made in the above proof is not true with respect to the Moussong metric, which explains the triangle-free hypothesis. Nevertheless, the above result seems likely to be true for an arbitrary large type Artin group, although we do not have an obvious proof to hand.

Lemma 26 Let $\Sigma, \Sigma^{\prime}$ denote minimal ciruits in $\Theta$. If $\Sigma \sim \Sigma^{\prime}$ then $\beta(\Sigma)=$ $\beta\left(\Sigma^{\prime}\right)$. Moreover, if $\Sigma \sim \Sigma^{\prime}$ in one step then the sequence $\left\{\Sigma_{i}\right\}_{i}$ in Definition 22 may be chosen so that $\beta\left(\Sigma_{i}\right)=\beta(\Sigma)$ for all $i$.

Proof It suffices to verify the statement concerning one step equivalence. We suppose therefore that, as in the definition of one step equivalence (Definition 22), there exists a sequence $\left\{\Sigma_{i}\right\}, i=0,1, . ., k$, of minimal circuits with $\Sigma_{0}=\Sigma$ and $\Sigma_{k}=\Sigma^{\prime}$, a subpath $(S, V, T)$ common to $\Sigma_{0}$ and $\Sigma_{k}$, and a sequence of subpaths $\left(S, V_{i}, T_{i}\right) \subset \Sigma_{i} \cap \Sigma_{i-1}$ with $V_{i} \neq V$ for $i=1, . ., k$. We shall write $\beta_{i}=\beta\left(\Sigma_{i}\right)$ for $i=0,1, . ., k$. We also let $e, e_{1}, . ., e_{k}$ denote the edges in $\Delta$ such that $V=\beta_{0} V_{e}$ and $V_{i}=\beta_{i} V_{e_{i}}$ for $i=1, . ., k$. Note that these edges are not necessarily distinct. Finally, we suppose that the sequence $\left\{\Sigma_{i}\right\}_{i}$ is chosen so as to minimise the length $k$. With this assumption we make the following claim:

Claim If $e_{i}=e_{j}$ for some $1 \leq i<j \leq k$ then $\beta_{i} \neq \beta_{j-1}$.

Proof Fix $j \in\{1, . ., k\}$, and set $e_{j}=\{s, t\}$. Then $\left(S, V_{j}, T_{j}\right)=\beta_{j}\left(F_{s}, V_{e_{j}}, F_{t}\right)$. Since this subpath is common to both $\Sigma_{j}$ and $\Sigma_{j-1}$ we have, by Lemma 24(iii), that $\beta_{j-1}^{-1} \beta_{j} \in\left\langle x_{e_{j}}\right\rangle$, and therefore that $\beta_{j-1}\left(V_{e_{j}}\right)=\beta_{j}\left(V_{e_{j}}\right)=V_{j}$ 
and $\beta_{j-1}\left(\left\{F_{s}, F_{t}\right\}\right)=\beta_{j}\left(\left\{F_{s}, F_{t}\right\}\right)=\left\{T_{j}, S\right\}$. Now suppose, by way of contradiction, that $e_{i}=e_{j}$ and $\beta_{i}=\beta_{j-1}$, for some $i<j$. Then, by the previous observation, we have $V_{i}=\beta_{i}\left(V_{e_{i}}\right)=\beta_{j-1}\left(V_{e_{j}}\right)=V_{j}$, and $\left\{T_{i}, S\right\}=\left\{T_{j}, S\right\}$ similarly. That is $T_{i}=T_{j}$ and $V_{i}=V_{j}$. But then one can simply remove the circuits $\Sigma_{i}, . ., \Sigma_{j-1}$ from the sequence to obtain a shorter sequence satisfying the one step equivalence of Definition 22, contrary to our choice of a shortest length sequence.

Applying Lemma 24 (iii), we have a sequence of elements $h_{i}=\beta_{i-1}^{-1} \beta_{i}$, for $i=1, \ldots, k$, such that $h_{i} \in\left\langle x_{e_{i}}\right\rangle$ for each $i$. Also, writing $h=\beta_{0}^{-1} \beta_{k}$ we have that $h \in\left\langle x_{e}\right\rangle$ (again by Lemma 24 (iii)) and

$$
h=h_{1} h_{2} h_{3} \cdots h_{k} .
$$

Note that by Lemma 25 the elements $x_{d}$ for $d \in E(\Delta)$ generate a free group $L$. The above Claim implies that deleting all trivial syllables $h_{i}=1$ from the expression $h_{1} h_{2} \ldots h_{k}$ yields a reduced form for the element $h$ with respect to the structure of $L$ as a free product of cyclic groups $\left\langle x_{d}\right\rangle$ for $d \in E(\Delta)$. (For, if $h_{i+1}=h_{i+2}=\cdots=h_{j-1}=1$ then $\beta_{i}^{-1} \beta_{j-1}=h_{i+1} \cdots h_{j-1}=1$ and so, by the Claim, $e_{i} \neq e_{j}$ which implies that $h_{i}, h_{j}$ do not belong to the same free factor). However, since $h$ lies in the free factor $\left\langle x_{e}\right\rangle$, it follows that either $h=1$ or there is a unique $r \in\{1, . ., k\}$ for which $h_{r}$ is nontrivial with $h_{r}=h$ and $e_{r}=e$. In the latter case we would have $\beta_{0}^{-1} \beta_{r}=h \in\left\langle x_{e}\right\rangle$ which would imply that $V_{r}=\beta_{r}\left(V_{e}\right)=\beta_{0}\left(V_{e}\right)=V$, a contradiction. Thus $h=1$, and consequently $h_{i}=1$, and so $\beta_{i}=\beta_{0}$, for all $i=1, . ., k$.

Lemma 27 Let $\Sigma, \Sigma^{\prime}$ denote minimal circuits in $\Theta$. If $\Sigma \sim \Sigma^{\prime}$ then $\Sigma$ and $\Sigma^{\prime}$ belong to the same chunk of $\Theta$.

Proof Suppose that $\Sigma \sim \Sigma^{\prime}$ by a one step equivalence as described in Definition 22] Without loss of generality we may suppose that $\Sigma \subset \widehat{\Delta}$. Then we have a sequence $\Sigma=\Sigma_{0}, \Sigma_{1}, . ., \Sigma_{k}=\Sigma^{\prime}$ of minimal circuits as in the definition where, by Lemma 26, we may suppose that all circuits $\Sigma_{i}$ lie in $\widehat{\Delta}$. Considering, for simplicity, all circuits as circuits in $\Delta$, we have that $\Sigma, \Sigma^{\prime}$ have an edge $e$ in common while, for each $i=1, . ., k$, the circuits $\Sigma_{i}$ and $\Sigma_{i-1}$ meet along an edge $e_{i}$ different from $e$. If $\Sigma$ and $\Sigma^{\prime}$ were to lie in distinct chunks then the edge $e$ would have to separate $\Delta$ into two pieces $A$ and $B$ containing $\Sigma$ and $\Sigma^{\prime}$ respectively. However, the fact that $e_{i} \neq e$ would then imply that $\Sigma_{i} \subset A$ if and only if $\Sigma_{i-1} \subset A$, and therefore that $\Sigma$ and $\Sigma^{\prime}$ both lie in $A$, a contradiction. Thus $\Sigma$ and $\Sigma^{\prime}$ lie in the same chunk. 


\subsection{Circuits in the same chunk are equivalent}

For this part of the proof it will be convenient to only consider circuits in the original defining graph $\Delta$ (before subdivision of the edges). We shall say that an edge or vertex $T$ of $\Delta$ separates subsets $A$ and $B$ if $\Delta$ can be written $\Delta_{1} \cup_{T} \Delta_{2}$ with $A \subset \Delta_{1}$ and $B \subset \Delta_{2}$. Note that this terminology implies that $T$ is a separating edge or vertex of $\Delta$.

Lemma 28 If $\Delta$ is a connected graph and $\Sigma, \Sigma^{\prime}$ are minimal circuits then either there is a vertex of $\Delta$ which separates them, or they are joined by a sequence $\Sigma=\Sigma_{0}, \Sigma_{1}, \Sigma_{2}, . ., \Sigma_{n}=\Sigma^{\prime}$ of minimal circuits of $\Delta$ such that $\Sigma_{i}$ and $\Sigma_{i-1}$ have an edge in common, for each $i=1, . ., n$.

Proof We first observe that there is necessarily a simple edge path in $\Delta$ whose first edge lies in $\Sigma$ and whose last edge lies in $\Sigma^{\prime}$. Moreover, since no vertex of this path can separate $\Sigma$ and $\Sigma^{\prime}$, we may suppose that no two subsequent edges along this path are separated in $\Delta$ by their common vertex. It will now suffice to show that if $e, e^{\prime}$ are edges of $\Delta$ with a common vertex that does not separate them then there exists a sequence of minimal circuits $\Sigma_{0}, \Sigma_{1}, . ., \Sigma_{n}$ such that $e \subset \Sigma_{0}, e^{\prime} \subset \Sigma_{n}$ and $\Sigma_{i} \cap \Sigma_{i-1}$ contains an edge for each $i=1, . ., n$. Suppose we have $e=\{t, s\}$ and $e^{\prime}=\left\{s, t^{\prime}\right\}$. Since $s$ does not separate the two edges, there must exist a simple path $\alpha$ from $t$ to $t^{\prime}$ which does not pass through $s$. Concatenating $\alpha$ with the edges $e, e^{\prime}$ yields a simple circuit $C$ through $e, e^{\prime}$. If $C$ is minimal then there is nothing left to prove. Otherwise, we may find a short-circuit which decomposes $C$ into circuits $C_{1}$ and $C_{2}$ of strictly shorter length. Either $e, e^{\prime}$ both still lie in the same circuit, $C_{1}$ say, in which case we replace $C$ with $C_{1}$, or $e \subset C_{1}$ and $e^{\prime} \subset C_{2}$ and there is an edge $e^{\prime \prime}=\left\{s, t^{\prime \prime}\right\}$ common to $C_{1}$ and $C_{2}$ and adjacent to $s$, in which case we replace $C$ with the sequence $C_{1}, C_{2}$. In either case, the desired result follows by induction on the length of $C$.

Lemma 29 If minimal circuits $\Sigma, \Sigma^{\prime}$ of a graph $\Delta$ meet along an edge $e$ then either e separates $\Sigma$ from $\Sigma^{\prime}$, or there exists a sequence $\Sigma=\Sigma_{0}, \Sigma_{1}, \Sigma_{2}, . ., \Sigma_{k}=$ $\Sigma^{\prime}$ of minimal circuits such that, for each $i=1, . ., k$, the circuits $\Sigma_{i}$ and $\Sigma_{i-1}$ have a common edge $e_{i}$ which is adjacent to but not equal to $e$.

Proof Let $e \in E(\Delta)$, and write $S(e)$ for the set of vertices of $\Delta$ which are not themselves endpoints of $e$, but which are adjacent along an edge to one or other endpoint of $e$. For $u, v$ distinct vertices in $S(e)$, we define a joining arc from $u$ to $v$, to be any simple edge-path from $u$ to $v$ in $\Delta$ which does not 
pass through either of the endpoints of $e$. If $\alpha$ is a joining arc from $u$ to $v$, then write $\Sigma(\alpha)$ for the simple circuit formed from $\alpha$ and the unique shortest edge-path (of length 2 or 3 ) from $u$ to $v$ passing through one or both of the endpoints of $e$. Note that joining arcs are thus in bijective correspondence with the simple closed circuits in $\Delta$ which intersect the edge $e$ (at one or both of its endpoints). The joining arc $\alpha$ shall be said to be minimal if $\Sigma(\alpha)$ is a minimal circuit.

Sublemma Let $e \in E(\Delta), u, v$ distinct vertices in $S(e)$, and $\alpha$ a joining arc from $u$ to $v$. Then there exists a sequence $u=u_{1}, u_{2}, . ., u_{k}=v$ of elements of $S(e)$ and minimal joining arcs $\alpha_{i}$ from $u_{i}$ to $u_{i+1}$, for $i=1, . ., k-1$.

Proof Suppose $\alpha$ is not minimal. Then $\Sigma(\alpha)$ admits a short circuit $\sigma$ which decomposes $\Sigma(\alpha)$ into two simple circuits $C_{1}$ and $C_{2}$ each of strictly smaller length than $\Sigma(\alpha)$. Either $\sigma$ is disjoint from $e$, or one of its endpoints is also an endpoint of $e$. In the first case, one of the two smaller circuits, $C_{1}$ say, contains $u, v$ and at least one vertex of $e$, and is thus equal to $\Sigma\left(\alpha^{\prime}\right)$ where $\alpha^{\prime}$ is a joining arc from $u$ to $v$. In the second case, both circuits $C_{1}$ and $C_{2}$ pass though a vertex of $e$ and we may suppose that $C_{1}$ contains $u$ and $C_{2}$ contains $v$. In fact, if $w$ denotes the interior vertex of $\sigma$ closest to $e$ (so that $w \in S(e)$ ), then $C_{1}=\Sigma\left(\alpha_{1}\right)$ where $\alpha_{1}$ is a joining arc from $u$ to $w$, and $C_{2}=\Sigma\left(\alpha_{2}\right)$ where $\alpha_{2}$ is a joining arc from $w$ to $v$. The Sublemma now follows by induction on the length of $\Sigma(\alpha)$.

We shall now complete the proof of Lemma 29. We have an edge $e$ which is common to minimal circuits $\Sigma$ and $\Sigma^{\prime}$. Suppose that $e$ does not separate $\Sigma$ and $\Sigma^{\prime}$. Take distinct vertices $u, v \in S(e)$ such that $u \in \Sigma$ and $v \in \Sigma^{\prime}$. since $e$ does not separate the two circuits, there is a joining arc from $u$ to $v$ and, by the Sublemma, a sequence $u=u_{1}, . ., u_{k}=v$ of vertices in $S(e)$, and minimal joining arcs $\alpha_{i}$ from $u_{i}$ to $u_{i+1}$, for $i=1, . ., k-1$. Write $\Sigma_{0}=\Sigma, \Sigma_{i}=\Sigma\left(\alpha_{i}\right)$, for $i=1, . ., k-1$, and $\Sigma_{k}=\Sigma^{\prime}$. Now, for each $i=1, . ., k, \Sigma_{i} \cap \Sigma_{i-1}$ contains at least one edge $e_{i}$ which is adjacent to $e$ and has the vertex $u_{i}$ as one of its endpoints. In particular $e_{i} \neq e$.

Lemma 30 Let $\Sigma, \Sigma^{\prime}$ denote minimal circuits in $\Theta$. If $\Sigma$ and $\Sigma^{\prime}$ belong to the same chunk of $\Theta$ then $\Sigma \sim \Sigma^{\prime}$.

Proof Without loss of generality we may suppose that $\Sigma$ and $\Sigma^{\prime}$ are fundamental minimal circuits lying in the same chunk of $\widehat{\Delta}$. We may also, for 
simplicity, consider these circuits as circuits of the graph $\Delta$. (Note that by Proposition 16 circuits which are minimal in $\Delta$, equivalently $\widehat{\Delta}$, are also minimal as circuits of $\Theta$ ).

Suppose firstly that $\Sigma$ and $\Sigma^{\prime}$ share an edge $e \in E(\Delta)$. Since $\Sigma, \Sigma^{\prime}$ lie in the same chunk of $\Delta$, e cannot separate them and so Lemma 29 gives a sequence of minimal circuits $\Sigma=\Sigma_{0}, \Sigma_{1}, \Sigma_{2}, \ldots, \Sigma_{n}=\Sigma^{\prime}$ such that each $\Sigma_{i}$ shares an edge $e_{i}$ with the previous and $e_{i}$ is adjacent to but distinct from $e$. Let $e=\{s, t\}$. If $e_{i} \cap e=\{s\}$ for all $i=1, . ., n$, then $\Sigma_{0} \sim \Sigma_{n}$ in one step. Suppose then that $e_{i} \cap e=\{s\}$, for $i=1, . ., k$, and $e_{k+1} \cap e=\{t\}$, for some $k<n$. Then $\Sigma_{k}$ contains the path $e_{k}, e, e_{k+1}$. It follows, since $e \subset \Sigma_{k}$, that $\Sigma_{0} \sim \Sigma_{k}$ in one step. By a straightforward induction we conclude that $\Sigma \sim \Sigma^{\prime}$.

More generally, if $\Sigma$ and $\Sigma^{\prime}$ lie in the same chunk of $\Delta$ then, since no vertex can separate them, Lemma 28 gives a sequence of minimal circuits $\Sigma=$ $\Sigma_{0}, \Sigma_{1}, \Sigma_{2}, \ldots, \Sigma_{n}=\Sigma^{\prime}$ in $\Delta$ where each shares an edge with the next. Moreover, since any two adjacent chunks meet along at most a single edge, we may suppose (by passing to a subsequence) that all circuits $\Sigma_{i}$ lie in the same chunk of $\Delta$. But, by the preceding argument, this means that $\Sigma_{i-1} \sim \Sigma_{i}$ for each $i$, and so $\Sigma \sim \Sigma^{\prime}$.

\section{Chunk rigidity in $\Theta_{W}$ the graph of fixed sets in $\mathbb{D}_{W}$}

We obtain results exactly analogous to those of the last two sections in the context of the action of a CLTTF Coxeter group $W=W(\Delta)$ on its Davis complex $\mathbb{D}_{W}$. Throughout this section we shall view $\mathbb{D}_{W}$ as a subcomplex of $\mathbb{D}$ via the map induced by the Tits section. We note that $\mathbb{D}_{W}$ is geodesically convex in $\mathbb{D}$ with respect to either the Moussong metric, or the cubical metric. We shall write $\bar{x}$ for the image in $W$ of an element $x \in G$, and $i_{W}: W \rightarrow G$ for the Tits section.

For $s \in V(\Delta)$ we set $H_{s}:=F_{s} \cap \mathbb{D}_{W}$. Then $H_{s}$ is the fixed set in $\mathbb{D}_{W}$ of the standard reflection $\bar{s}$, and may be thought of as a "hyperplane" in the Davis complex. Note that $H_{s}$ is infinite if and only if $F_{s}$ is unbounded.

Definition We define the following sets

$$
\begin{aligned}
& \mathcal{V}_{W}=\left\{\text { singletons }\left\{w V_{e}\right\}: w \in W, e \in E(\Delta)\right\}, \text { and } \\
& \mathcal{F}_{W}=\left\{\text { infinite hyperplanes } w H_{s}: w \in W, s \in V(\Delta)\right\} .
\end{aligned}
$$

We define the graph $\Theta_{W}$ to be the bipartite graph with vertex set $\mathcal{V}_{W} \cup \mathcal{F}_{W}$ and edges $(V, H)$ whenever $V \in \mathcal{V}_{W}, H \in \mathcal{F}_{W}$ and $V \subset H$. 
The elements of $\mathcal{V}_{W}$ are characterized as the fixed sets of the maximal finite subgroups of $W$, namely the conjugates of dihedral groups $\langle\bar{s}, \bar{t}\rangle=\operatorname{Stab}\left(V_{e}\right)$ for all edges $e=\{s, t\} \in E(\Delta)$. Any two maximal finite subgroups $D_{1}$ and $D_{2}$ in $W$ intersect nontrivially if and only if their fixed points lie on a common hyperplane $w H_{s}$ for some $s \in V(\Delta)$ and $w \in W$, in which case $D_{1} \cap D_{2}=$ $\left\{1, w \bar{s} w^{-1}\right\}$. This condition is also equivalent to the statement that $\operatorname{Fix}\left(D_{1}\right)$ and $\operatorname{Fix}\left(D_{2}\right)$ both lie in $g F_{s}$ where $g=i_{W}(w)$ is the image of $w$ under the Tits section $W \hookrightarrow G$. Thus we have the following.

\section{Lemma 31}

(i) Any isomorphism $\phi: W \rightarrow W^{\prime}$ induces a graph isomorphism $\Phi: \Theta_{W} \rightarrow$ $\Theta_{W}^{\prime}$ such that, for all $X \in \mathcal{V}_{W} \cup \mathcal{F}_{W}$, we have $\operatorname{Stab}(\Phi(X))=\phi(\operatorname{Stab}(X))$. Moreover, this is a " $\mathcal{V} \mathcal{F}_{W}$-isomorphism": $\Phi\left(\mathcal{V}_{W}\right)=\mathcal{V}_{W}^{\prime}$ and $\Phi\left(\mathcal{F}_{W}\right)=$ $\mathcal{F}_{W}^{\prime}$.

(ii) The inclusion of $\mathbb{D}_{W}$ into $\mathbb{D}$ induces a natural inclusion $\Theta_{W} \hookrightarrow \Theta$ (such that $\left\{w V_{e}\right\} \mapsto\left\{i_{W}(w) V_{e}\right\}$ and $\left.w H_{s} \mapsto i_{W}(w) F_{s}\right)$.

We shall identify $\Theta_{W}$ with its image in $\Theta$ under the map of part (ii) of the Lemma. Note that the fundamental subgraph $\widehat{\Delta}$ lies inside $\Theta_{W}$, ie, $\widehat{\Delta} \subset$ $\Theta_{W} \subset \Theta$. Note also that any graph isomorphism $\Phi: \Theta_{W} \rightarrow \Theta_{W}^{\prime}$ will respect the family of circuits of $\Theta_{W}$ which are minimal as circuits of $\Theta_{W}$.

Lemma 32 Let $\Sigma$ denote a simple circuit in $\Theta_{W}$. Then $\Sigma$ is minimal as a circuit of $\Theta_{W}$ if and only if it is minimal as a circuit of $\Theta$. In particular, the minimal circuits of $\Theta_{W}$ are precisely the translates of minimal circuits of the fundamental subgraph $\widehat{\Delta}$ by elements of $W$.

Proof In Section [6. Proposition [15] was proved by showing that if a circuit $\Sigma$ fails to lie in a fundamental region of $\Theta$ then it admits a short circuit, and the only short circuits exhibited throughout the proof were constructed from one or more chords of the polygon $\bar{\Sigma}$ (cf Lemma 19] and Figure 2). If we are given $\Sigma_{W}$ a simple circuit in $\Theta_{W}$ then, since $\mathbb{D}_{W}$ is a geodesically convex subcomplex of $\mathbb{D}$, the simple closed curve $\bar{\Sigma}_{W}$ and any chord of $\bar{\Sigma}_{W}$ are contained in $\mathbb{D}_{W}$. It follows that any short circuit produced in the proof of Proposition 15] is a short circuit in $\Theta_{W}$. Thus any circuit which is minimal as a circuit of $\Theta_{W}$ is basic. Note that a circuit in $\widehat{\Delta}$ which is minimal in $\Theta_{W}$ is necessarily minimal in $\widehat{\Delta}$ and therefore, by Proposition [16 minimal in $\Theta$. It follows that any circuit which is minimal in $\Theta_{W}$ is minimal in $\Theta$. The converse is obvious. 
We may now define an equivalence relation $\sim_{W}$ on the set of minimal circuits of $\Theta_{W}$ exactly as per Definition 22. but with reference only to the minimal circuits of $\Theta_{W}$. Clearly, this equivalence relation is respected by any isomorphism of $\Theta_{W}$. Let $\Sigma, \Sigma^{\prime}$ be minimal circuits of $\Theta_{W}$. Then it follows immediately from Lemma [26] that $\Sigma \sim_{W} \Sigma^{\prime}$ if and only if $\Sigma \sim \Sigma^{\prime}$ (as circuits in $\Theta$ ). Thus, by Proposition [23, we have that $\Sigma \sim_{W} \Sigma^{\prime}$ if and only if $\Sigma$ and $\Sigma^{\prime}$ lie in the same chunk of $\Theta_{W}$ where, by chunk of $\Theta_{W}$, we understand any translate of a chunk of the fundamental subgraph $\widehat{\Delta}$ by an element of $W$. The following is an immediate consequence of this statement and Lemma 31(i) above:

Proposition 33 Let $W=W(\Delta)$ and $W^{\prime}=W\left(\Delta^{\prime}\right)$ be CLTTF Coxeter groups. Any isomorphism $\phi: W \rightarrow W^{\prime}$ naturally induces a $\mathcal{V} \mathcal{F}_{W}$-isomorphism $\Theta_{W} \rightarrow \Theta_{W}^{\prime}$ which maps the solid chunks of $\Theta_{W}$ bijectively onto the solid chunks of $\Theta_{W}^{\prime}$.

\section{Automorphisms - Theorems 1, 2, and 4}

Let $\Delta, \Delta^{\prime} \in \mathcal{G}$ be CLTTF defining graphs, and write $G=G(\Delta), G^{\prime}=G\left(\Delta^{\prime}\right)$. We shall also simply write $\Theta$ and $\Theta^{\prime}$ for the corresponding fixed set graphs.

Lemma 34 Let $\varphi: G \rightarrow G^{\prime}$ be an isomorphism. Then:

(i) $\varphi$ induces a graph isomorphism $\Phi: \Theta \rightarrow \Theta^{\prime}$ such that $\Phi(\operatorname{Fix}(C))=$ $\operatorname{Fix}(\varphi(C))$ for any $C N V A$ subgroup $C<G$. The isomorphism $\Phi$ is a $\mathcal{V} \mathcal{F}$-isomorphism.

(ii) $\varphi$ induces a label preserving bijection $\bar{\varphi}: E(\Delta) \rightarrow E\left(\Delta^{\prime}\right)$ which is defined uniquely such that $G(\bar{\varphi}(e))$ and $\varphi(G(e))$ are conjugate subgroups of $G^{\prime}$ for each $e \in E(\Delta)$.

Proof (i) The fact that $\Phi$ is well-defined is just a restatement of Proposition 13. To see that $\Phi$ is necessarily a $\mathcal{V} \mathcal{F}$-isomorphism, observe that maximal CNVA subgroups conjugate to $\langle s\rangle$ for $s \in V(\Delta)$ are distinguished from those conjugate to $\left\langle z_{e}\right\rangle$ for $e \in E(\Delta)$ by the fact that a standard generator $s$ is a primitive element of $G$, while $z_{e}$ is not (if $e=\{s, t\}$ then $z_{e}=(s t)^{k}$ where $\left.k=\operatorname{lcm}\left(m_{e}, 2\right) / 2\right)$.

(ii) For each $e \in E(\Delta)$ we have that $\Phi\left(V_{e}\right)=g_{e} V_{e^{\prime}}$ for some $g_{e} \in G^{\prime}$ and $e^{\prime} \in E\left(\Delta^{\prime}\right)$, and therefore (since $\operatorname{Stab}\left(V_{e}\right)=G(e)$, etc) $\varphi(G(e))=g_{e} G\left(e^{\prime}\right) g_{e}^{-1}$. It follows from the description of the Deligne complex that rank 2 vertices $V_{e}$ 
and $V_{f}$ lie in distinct orbits of the group action unless $e=f$, and therefore that the stabilizers $G(e)$ and $G(f)$ are non-conjugate unless $e=f$. Therefore, setting $\bar{\varphi}(e)=e^{\prime}$ gives a well-defined bijection $\bar{\varphi}: E(\Delta) \rightarrow E\left(\Delta^{\prime}\right)$. Moreover, since groups $G(e)$ and $G\left(e^{\prime}\right)$ (for any edges $e$ and $e^{\prime}$ ) are non-isomorphic unless $m_{e}=m_{e^{\prime}}$ the bijection $\bar{\varphi}$ must be label preserving.

Note that the proof of the above Lemma depends heavily on the fact that $\varphi$ is an isomorphism, rather than an abstract commensurator.

Rcall that any Artin group $G(\Delta)$ admits a length homomorphism $\ell: G(\Delta) \rightarrow \mathbb{Z}$ such that $\ell(s)=1$ for each generator $s \in V(\Delta)$.

Lemma 35 Let $e=\{s, t\} \in E(\Delta), m_{e} \geq 3$, and suppose that $\alpha \in \operatorname{Aut}(G(e))$ such that $\ell(\alpha(s))=\ell(\alpha(t))=1$. Then $\alpha$ differs by an inner automorphism of $G(e)$ from either the identity or the graph automorphism $\tau: s \leftrightarrow t$.

Proof This is an easy consequence of the computation of the automorphism group of a dihedral type Artin group first performed in [12] (see also [8] for a description of the same automorphism group).

Lemma 36 (Chunk Invariance) Let $\varphi: G \rightarrow G^{\prime}$ be an isomorphism and suppose that $\ell(\varphi(s))=1$ for every generator $s \in V(\Delta)$ of $G$. Then, for each chunk $A$ of $\Delta$ (solid or otherwise), there exists a chunk $A^{\prime}$ of $\Delta^{\prime}$, a labelled graph isomorphism $\tau_{A}: A \rightarrow A^{\prime}$, and an element $g_{A} \in G^{\prime}$ such that the restriction $\varphi_{A}$ of $\varphi$ to the subgroup $G(A)$ is given by

$$
\varphi_{A}=g_{A} \circ \tau_{A}
$$

(where $g_{A}$ denotes conjugation by $g_{A}$ and, by abuse of notation, $\tau_{A}$ denotes the group isomorphism induced by the graph isomorphism $\tau_{A}$ ). Note that the mapping $A \mapsto A^{\prime}$ defines a bijection between the chunks of $\Delta$ and those of $\Delta^{\prime}$.

Proof First suppose that $A$ is a solid chunk of $\Delta$. We may equally view $A$ as a solid chunk of $\widehat{\Delta}$. Proposition 23] states that $\Phi$, the induced $\mathcal{V} \mathcal{F}$-isomorphism of Lemma 34(i), carries solid chunks of $\Theta$ to solid chunks of $\Theta^{\prime}$. That is to say that $\Phi(A)=g_{A}\left(A^{\prime}\right)$ for some $g_{A} \in G^{\prime}$ and some solid fundamental chunk $A^{\prime}$ of $\widehat{\Delta}^{\prime}$. In fact, the element $g_{A}$ and the chunk $A^{\prime}$ are uniquely determined. Now, for each edge $e \subset A$, we have $\varphi(G(e))=g_{A} G\left(e^{\prime}\right) g_{A}^{-1}$ (and $\Phi\left(V_{e}\right)=g_{A} V_{e^{\prime}}$ ) for some $e^{\prime} \subset A^{\prime}$. Moreover, if $e$ and $f$ are edges of $A$ and $e \cap f=\{s\}$ then $\varphi(\langle s\rangle)=g_{A}\left\langle s^{\prime}\right\rangle g_{A}^{-1}$ where $e^{\prime} \cap f^{\prime}=\left\{s^{\prime}\right\}$ (since $\langle s\rangle=G(e) \cap G(f)$ ). In fact $\varphi(s)=g_{A} s^{\prime} g_{A}^{-1}$, since we suppose that $\ell(\varphi(s))=1$. Note also that 
$e^{\prime}=\bar{\varphi}(e)$, where $\bar{\varphi}: E(\Delta) \rightarrow E\left(\Delta^{\prime}\right)$ is the induced bijection of Lemma 34(ii). It now follows that the restriction of $\bar{\varphi}$ to the edges of $A$ determines a graph isomorphism $\tau_{A}$ and that $\varphi_{A}=g_{A} \circ \tau_{A}$.

The case where $A=e$ is a non-solid chunk follows easily by taking $A^{\prime}=\bar{\varphi}(e)$ and applying Lemma 35 .

\subsection{Proof of Theorem 1}

We suppose that we are given a group isomorphism $\varphi: G \rightarrow G^{\prime}$. Our approach will be to compose this isomorphism with known isomorphisms of types (2)-(4) (inversions, Dehn twist and inner automorphisms and edge twist isomorphisms) until it is reduced to a graph automorphism (type (1)).

Applying the inversion automorphisms We shall use the existence of the standard length homomorphism $\ell: G^{\prime} \rightarrow \mathbb{Z}$ (such that $\ell(s)=1$ for all $s \in$ $\left.V\left(\Delta^{\prime}\right)\right)$. For each $e \in E(\Delta)$, we have $\varphi\left(\left\langle z_{e}\right\rangle\right)=g\left\langle z_{e^{\prime}}\right\rangle g^{-1}$, where $e^{\prime}=\bar{\varphi}(e)$ and $g \in G^{\prime}$. Also, since $\ell\left(z_{e^{\prime}}\right) \neq 0$, the element $z_{e^{\prime}}$ is not conjugate to its inverse. Thus we have a well-defined function $\nu: E(\Delta) \rightarrow\{ \pm 1\}$ such that $\varphi\left(z_{e}\right) \sim z_{e^{\prime}}^{\nu(e)}$. If $e=\{s, t\}$ where both $\langle s\rangle$ and $\langle t\rangle$ are CNVA then both $\varphi(s)$ and $\varphi(t)$ have absolute length 1 (since $s$ and $t$ are mapped to generators of maximal CNVA subgroups). Since $\varphi$ respects the relation $(s t)^{k}=z_{e}\left(k=\operatorname{lcm}\left(m_{e}, 2\right) / 2\right)$, we must therefore have $\ell(\varphi(s))=\ell(\varphi(t))=\nu(e)$. This argument applies to every edge of $\Delta$ with the exception of the even labelled terminal edges, where the terminal generator does not generate a CNVA subgroup. By connectedness of $\Delta$ it follows that $\nu$ is constant on the set $E(\Delta) \backslash\{$ even labelled terminal edges $\}$. By precomposing $\varphi$ with leaf inversions and a global inversion, as necessary, we may now suppose that $\nu(e)=1$ for all $e \in E(\Delta)$ and that $\ell(\varphi(s))=1$ for every CNVA generator $s \in V(\Delta)$. (Recall that if $\mu_{e}$ is a leaf inversion then it fixes all CNVA generators and $\left.\mu_{e}\left(z_{e}\right)=z_{e}^{-1}\right)$. Note that, for any edge $e=\{s, t\}$ the relation $(s t)^{k}=z_{e}$ implies that if $\ell(\varphi(s))=\nu(e)=1$ then $\ell(\varphi(t))=1$. Thus, it actually follows that $\ell(\varphi(s))=1$ for every single generator $s \in V(\Delta)$.

(In the last statement we are implicitly using the assumption that $\Delta$ has at least 3 vertices and is connected (C), and so has at least one CNVA generator).

Applying edge twists and Dehn twists We suppose from now on that $\ell(\varphi(s))=1$ for all $s \in V(\Delta)$. Let $B$ denote any connected subgraph of $\Delta$ which is a union of chunks. We shall show, by induction on the number of 
chunks in $B$, that we may arrange (by composing with isomorphisms of type (3) and (4)) that the restriction $\varphi_{B}$ of $\varphi$ to $G(B)$ is induced by a labelled graph isomorphism $\tau_{B}: B \rightarrow B^{\prime}$, for some connected subgraph $B^{\prime}$ of $\Delta^{\prime}$ (which is also necessarily a union of chunks of $\Delta^{\prime}$ ). In the case where $B=\Delta$ we have $\Delta=\Delta^{\prime}$ and $\varphi=\tau_{B}$ a graph automorphism of $G(\Delta)$, completing the proof of Theorem 1 .

If we take $B$ to be any single chunk then, by Lemma 36, we may suppose, up to an inner automorphism of $G^{\prime}$, that $g_{B}=1$ and $\varphi_{B}=\tau_{B}$.

Suppose now that the statement is already proven for some subgraph $B \neq \Delta$, and that $A$ is a chunk of $\Delta$ which does not lie in $B$ but which intersects $B$ nontrivially. We shall prove the statement for $A \cup B$. We have $\varphi_{B}=\tau_{B}$ for some graph isomorphism $\tau_{B}: B \rightarrow B^{\prime}$ and, by Lemma [36] we may suppose that $\varphi_{A}=g_{A} \circ \tau_{A}$, for some $g_{A} \in G^{\prime}$ and $\tau_{A}: A \rightarrow A^{\prime}$ where $A^{\prime}$ denotes a chunk of $\Delta^{\prime}$. There are two cases to consider.

Suppose firstly that $A$ and $B$ intersect along an edge $e=\{s, t\}$. Then $\varphi_{A}=$ $g_{A} \circ \tau_{A}$ and $\varphi_{B}=\tau_{B}$ must agree on the subgroup $G(e)$. In particular $\tau_{A}(e)=$ $\tau_{B}(e)=e^{\prime}=\left\{s^{\prime}, t^{\prime}\right\}$, and $g_{A}\left(\left\{s^{\prime}, t^{\prime}\right\}\right)=\left\{s^{\prime}, t^{\prime}\right\}$. It follows that $g_{A}$ lies in $G\left(e^{\prime}\right)$ (since $g_{A} G\left(e^{\prime}\right) g_{A}^{-1}=G\left(e^{\prime}\right)$ implies that $g_{A}\left(V_{e^{\prime}}\right)=V_{e^{\prime}}$ and so $g_{A} \in G\left(e^{\prime}\right)$ ). More precisely $g_{A}$ lies in the quasi-centre $\left\langle x_{e^{\prime}}\right\rangle$ of $G\left(e^{\prime}\right)$. By composing with a sequence of edge twist isomorphisms we may now suppose that $g_{A}=1$, and consequently that $\tau_{A}$ and $\tau_{B}$ agree on $e$ (ie, $\tau_{A}(s)=\tau_{B}(s)=s^{\prime}$ etc). But then $\varphi_{A \cup B}$ is induced from a labelled graph isomorphism, as required. (Note that each of the above edge twists will change $\Delta^{\prime}$ by a twist equivalence. However, they will compose to give a genuine Dehn twist in the case where the given $g_{A}$ is central in $\left.G\left(e^{\prime}\right)\right)$.

Now suppose that $A \cap B=\{s\}$, for some $s \in V(\Delta)$. Write $s^{\prime}, t^{\prime}$ for the elements of $V\left(\Delta^{\prime}\right)$ such that $\tau_{A}(s)=s^{\prime}$ and $\tau_{B}(s)=t^{\prime}$. Since $g_{A} \circ \tau_{A}(s)=\tau_{B}(s)$ we have that $g_{A} s^{\prime} g_{A}^{-1}=t^{\prime}$. We may now find a simple edge path $\gamma$ from $s^{\prime}$ to $t^{\prime}$ which consists of only odd label edges. (If not $s^{\prime}$ and $t^{\prime}$ would map to distinct cyclic factors of the abelianisation of $G^{\prime}$ and hence could not be conjugate in $\left.G^{\prime}\right)$.

Note that two chunks of the graph $\Delta$ are separated by a vertex if and only if they cannot be joined by a sequence of chunks where each has a edge in common with the next. Since $\bar{\varphi}: E(\Delta) \rightarrow E\left(\Delta^{\prime}\right)$ is a bijection which preserves chunks it also preserves the above property. Therefore, since $A$ is separated from any chunk in $B$ by the vertex $s$, it follows that $A^{\prime}=\tau_{A}(A)$ is separated from at least one chunk of $B^{\prime}=\tau_{B}(B)$ by some vertex $v^{\prime} \in V\left(\Delta^{\prime}\right)$. (Note that both graph isomorphisms $\tau_{A}$ and $\tau_{B}$ are induced by $\left.\bar{\varphi}\right)$. Since $v^{\prime}$ separates $A^{\prime}$ from 
one chunk in $B^{\prime}$, and $B^{\prime}$ is connected, it must separate $A^{\prime}$ from every chunk of $B^{\prime}$, and hence from $B^{\prime}$ itself. Moreover, the path $\gamma$ must pass through the vertex $v^{\prime}$ separating $A^{\prime}$ from $B^{\prime}$. We may thus write

$$
\Delta^{\prime}=\Delta_{1}^{\prime} \bigcup_{v_{1}^{\prime}=v^{\prime}=v_{2}^{\prime}} \Delta_{2}^{\prime}
$$

where $A^{\prime} \subset \Delta_{1}^{\prime}$ and $B^{\prime} \subset \Delta_{2}^{\prime}$. We also decompose $\gamma$ into a union of subpaths $\gamma_{1}$ from $s^{\prime}$ to $v_{1}^{\prime}$ in $\Delta_{1}$ and $\gamma_{2}$ from $v_{2}^{\prime}$ to $t^{\prime}$ in $\Delta_{2}$ where the endpoints $v_{1}^{\prime}$, $v_{2}^{\prime}$ are identified to the vertex $v^{\prime}$. Now, by applying a sequence of edge twist isomorphisms to $G^{\prime}$ (along the edges of the subpath $\gamma_{2}$ in $\Delta_{2}^{\prime}$ ), we may modify $\Delta^{\prime}$ to the graph

$$
\Delta_{1}^{\prime} \bigcup_{v_{1}^{\prime}=t^{\prime}} \Delta_{2}^{\prime}
$$

By a similar sequence of edge twists (following the subpath $\gamma_{1}$ from $v_{1}^{\prime}$ back to $s^{\prime}$ in $\Delta_{1}$ ), we may now further modify this graph to the graph

$$
\Delta_{1}^{\prime} \bigcup_{s^{\prime}=t^{\prime}} \Delta_{2}^{\prime}
$$

Note that the above edge twists may be chosen to be the identity on $G(B)$. Composing with these edge twists therefore alters the isomorphism $\varphi: G \rightarrow G^{\prime}$ so that $\varphi_{B}=\tau_{B}$ is unchanged and $\varphi_{A}=g_{A} \circ \tau_{A}$ for a (possibly different) $g_{A}$ and $\tau_{A}$ satisfying $\tau_{A}(s)=\tau_{B}(s)=s^{\prime}$ and $g_{A} s^{\prime} g_{A}^{-1}=s^{\prime}$. But that is to say that $g_{A} \in C_{G^{\prime}}\left(\left\langle s^{\prime}\right\rangle\right)$, and so, by applying a Dehn twist automorphism to $G^{\prime}$, we may suppose that $g_{A}=1$. At this point the restriction of $\varphi$ to $G(A \cup B)$ is simply induced by a labelled graph isomorphism, as required. This completes the proof of Theorem 1 .

\subsection{Proof of Theorem 2}

The nontrivial content of Theorem 2 is contained in the statement that

$$
\operatorname{ker}(\pi, \Delta)=\operatorname{Pure}(\Delta) \rtimes \operatorname{Inv}(\Delta) .
$$

To prove this we simply repeat the proof of Theorem 1 above with the added assumptions that $\Delta^{\prime}=\Delta$ and that the map $\bar{\varphi}$ induced on edges is the identity. One observes that only inversions and Dehn twist isomorphisms are needed to complete the proof, and the statement of Theorem 2 follows. 


\subsection{Proof of Theorem 4}

To establish Theorem 4 it suffices to repeat once again the arguments of Theorems 1 and 2 replacing the isomorphism $\varphi: G \rightarrow G^{\prime}$ with an isomorphism $\phi: W \rightarrow W^{\prime}$ between the corresponding Coxeter groups, and using Proposition 33 in the place of Propositions 13 and 23. We also make the following observations:

- The fact that $\phi$ induces a well defined bijection $\bar{\phi}: E(\Delta) \rightarrow E(\Delta)$ follows by consideration of the action on the Davis complex, and the fact that $\bar{\phi}$ respects the labelling follows from the fact that $W(e)$, being a dihedral group of order $2 m_{e}$, is distinguished up to isomorphism by the label $m_{e}$.

- The consideration of inversion automorphisms does not appear in the Coxeter group situation, but is replaced with a consideration of dihedral twist automorphisms. In place of Lemma 35 we make the following observations. Let $e=\{s, t\} \in E(\Delta)$ and $m=m_{e} \geq 3$. Then the dihedral group $W(e) \cong D_{2 m}$ has presentation $\left\langle t, \rho \mid t^{2}=\rho^{m}=(\rho t)^{2}=1\right\rangle$, where $\rho=s t$. The reflections (conjugates of $s$ and $t$ ) are characterized as the primitive involutions of $W(e)$. Therefore, any automorphism of $W(e)$ can be modified by an inner automorphism and, if necessay, by the graph automorphism exchanging $s$ and $t$ so that it fixes the generator $t$ say. The cyclic subgroup generated by $\rho$ is characteristic. So any automorphism of $W(e)$ which fixes the generator $t$ is given by $t \mapsto t$ and $\rho \mapsto \rho^{k}$ where $k$ represents a unit of the ring $\mathbb{Z} / m \mathbb{Z}$. Equivalently $t \mapsto t$ and $s \mapsto(s t)^{r} s(s t)^{-r}$ where $2 r+1 \equiv k(m)$. That is to say that any automorphism of $W(e)$ differs from a dihedral twist automorphism by a composition of inner and graph automorphisms. This establishes the analogue of Lemma 36 in the case of a non-solid chunk $A=e$.

\section{0 "Vertex links" in $\Theta$ and rank 2 vertices of the Deligne complex}

In order to arrive finally at a proof of Theorem 3 we need to pursue a little further our study of the graph $\Theta$ so as to establish a rigidity property which is closely associated with the structure of links of rank 2 vertices in the Deligne complex. We suppose throughout this section that $\Delta$ is a CLTTF defining graph and $\Theta$ the associated graph of fixed sets in the Deligne complex $\mathbb{D}$. 
Let $\operatorname{Chk}(\Theta):=\{$ solid chunks of $\Theta\}$, and $\operatorname{Chk}(\widehat{\Delta}):=\{$ solid chunks of $\widehat{\Delta}\}$. By virtue of Proposition 23 and Lemma 26 there exist well-defined maps

$$
\beta: \operatorname{Chk}(\Theta) \rightarrow G \text { and fund: } \operatorname{Chk}(\Theta) \rightarrow \operatorname{Chk}(\widehat{\Delta})
$$

such that $X=\beta(X)$.fund $(X)$, for each solid chunk $X$ in $\Theta$.

Definition (Oriented solid chunks) Let $G=G(\Delta)$ be a CLTTF Artin group, and $\Theta$ the associated graph of fixed sets. Fix $V \in \mathcal{V}$ and let $X$ be a solid chunk of $\Theta$ containing $V$. By an orientation of $X$ we mean a choice of vertex $F \in X$ adjacent to $V$ (necessarily, $F \in \mathcal{F}$ ). We write $\mathbf{X}=(X, V, F)$ for the oriented chunk based at $V$ with orientation given by $F$. Note that there are always exactly two choices of orientation for a chunk $X$ based at $V$. Namely, writing $V=\beta(X) V_{e}$ for some edge $e=\{s, t\} \in E(\Delta)$, we have orientations given by $\beta(X) F_{s}$ and $\beta(X) F_{t}$. We shall denote by $-\mathbf{X}$ the chunk $\mathbf{X}$ with the opposite orientation.

We say that two oriented solid chunks $\mathbf{X}_{1}=\left(X_{1}, V, F_{1}\right)$ and $\mathbf{X}_{2}=\left(X_{2}, V, F_{2}\right)$ based at $V$ in $\Theta$ are equivalent, written $\mathbf{X}_{1} \simeq \mathbf{X}_{2}$, if $\mathbf{X}_{2}=g \mathbf{X}_{1}$ for some $g$ in the pointwise stabilizer of $F_{1}$ under the action of $G$. In other words, $\left(X_{1}, V, F_{1}\right) \simeq$ $\left(X_{2}, V, F_{2}\right)$ if and only if, $\beta\left(X_{1}\right)^{-1} \beta\left(X_{2}\right) \in\langle s\rangle$ and $F_{1}=F_{2}=\beta F_{s}$, for some $s \in V(\Delta)$. (Here $\beta$ may be any element of the coset $\beta\left(X_{1}\right) \operatorname{Stab}\left(F_{s}\right)$ of the setwise stabilizer of $F_{s}$ under the action of $G$ ).

Note that the pointwise stabilizer of a fixed tree $F \in \mathcal{F}$ is strictly smaller than its setwise stabilizer. Thus the equivalence class of an oriented solid chunk $(X, V, F)$ is not determined just by the pair $(V, F)$. We can however characterize the above equivalence relation purely in terms of the structure of $\Theta$. Given an oriented solid chunk $\mathbf{X}=(X, V, F)$ we define the set

$$
N(\mathbf{X})=\{U \in \mathcal{V}: U \in X, U \neq V \text { and } U \text { is adjacent to } F\} .
$$

Note that $N(\mathbf{X})$ and $N(-\mathbf{X})$ are disjoint nonempty sets.

Lemma 37 Let $\mathbf{X}_{i}=\left(X_{i}, V, F_{i}\right)$ denote oriented chunks based at $V$, for $i=1,2$. Then the following are equivalent:

(1) $N\left(\mathbf{X}_{1}\right)=N\left(\mathbf{X}_{2}\right)$;

(2) $N\left(\mathbf{X}_{1}\right) \cap N\left(\mathbf{X}_{2}\right) \neq \emptyset$;

(3) $\quad \mathbf{X}_{1} \simeq \mathbf{X}_{2}$. 
Proof Clearly $(1) \Longrightarrow(2)$, and $(3) \Longrightarrow(1)$ because any element of the pointwise stabilizer of $F_{1}$ fixes every vertex of $\Theta$ adjacent to $F_{1}$ (since $U \in \mathcal{V}$ is adjacent to $F_{1}$ if and only if $U \subset F_{1}$ ). We shall show (2) $\Longrightarrow$ (3). Suppose $U \in N\left(\mathbf{X}_{1}\right) \cap N\left(\mathbf{X}_{2}\right)$. Then $F_{1}=F_{2}$ and $X_{1}$ and $X_{2}$ share the path $\left(V, F_{1}, U\right)$. Statement (3) now follows from Lemma 24](ii).

Definition (The link graph $L(V, \Theta)$ ) For each $V \in \mathcal{V}$ we define $L(V, \Theta)$ to be the graph with vertices the equivalence classes of oriented solid chunks based at $V$, and an edge for each solid chunk $X$ containing $V$, the endpoints of which are determined by the two possible orientations of $X$ based at $V$. Thus, for each pair $\{\mathbf{X},-\mathbf{X}\}$ of oriented solid chunks at $V$, there is a single edge in $L(V, \Theta)$ whose vertices are the just the equivalence classes of $\mathbf{X}$ and $-\mathrm{X}$ respectively.

Let $A$ denote a solid chunk of $\widehat{\Delta}$. We shall write $L_{A}(V, \Theta)$ for the subgraph of $L(V, \Theta)$ consisting of those edges associated to solid chunks in the same $G$-orbit as $A$ (ie, solid chunks $X$ such that $\operatorname{fund}(X)=A$ ).

Note that the graph $L_{A}(V, \Theta)$ may often be empty, and will be non-empty if and only if some translate of the fundamental chunk $A$ contains $V$. It is also possible that $V$ lies in no solid chunk whatsoever, in which case the whole graph $L(V, \Theta)$ is empty. This happens at every vertex $V \in \mathcal{V}$ whenever $\Delta$ is a tree.

The following Proposition shows that the vertex link graph just defined is, on the one hand, canonical (with respect to $\mathcal{V F}$-isomorphism) and, on the other hand, strongly tied to the structure of the Deligne complex.

Proposition 38 Let $\Delta, \Delta^{\prime}$ denote CLTTF defining graphs and $\Theta=\Theta(\Delta)$ and $\Theta^{\prime}=\Theta\left(\Delta^{\prime}\right)$ the associated fixed set graphs. Let $V \in \Theta$ denote a type $\mathcal{V}$ vertex.

(i) Any $\mathcal{V} \mathcal{F}$-isomorphism $\Phi: \Theta \rightarrow \Theta^{\prime}$ induces a well-defined graph isomorphism

$$
\Phi_{V}: L(V, \Theta) \rightarrow L\left(\Phi(V), \Theta^{\prime}\right)
$$

such that the vertex of $L(V, \Theta)$ represented by a based oriented solid chunk $(X, V, F)$ is mapped under $\Phi_{V}$ to the vertex of $L\left(\Phi(V), \Theta^{\prime}\right)$ represented by $(\Phi(X), \Phi(V), \Phi(F))$.

(ii) The graph $L(V, \Theta)$ is a disjoint union of the subgraphs $L_{A}(V, \Theta)$, for solid chunks $A \subset \widehat{\Delta}$, and each nontrivial component $L_{A}(V, \Theta)$ is naturally isomorphic to $\operatorname{Lk}(V, \mathbb{D})$, the link of $V$ in the Deligne complex. 
Proof (i) Since, by Proposition 23, the $\mathcal{V} \mathcal{F}$-isomorphism $\Phi$ respects solid chunks, it will also respect oriented solid chunks mapping the set $N(X, V, F)$ onto the set $N(\Phi(X), \Phi(V), \Phi(F))$. By Lemma 37] it follows that the equivalence relation $\simeq$ is preserved under the $\mathcal{V} \mathcal{F}$-isomorphism. The map $\Phi_{V}$ is therefore well-defined, and clearly a graph isomorphism.

(ii) Let $e=\{s, t\} \in E(\Delta)$ denote the edge of $\Delta$ such that $V$ is a translate of $V_{e}$. Then the link $\operatorname{Lk}(V, \mathbb{D})$ of the rank 2 vertex in $\mathbb{D}$ may be described as follows. For each $g \in G$ such that $g V_{e}=V$ (ie, such that $V \subset g K$ ), there is exactly one edge in $L k(V, \mathbb{D})$ contributed by the translate $g K$ of the fundamental region $K$. This edge has endpoints corresponding to the edges $\left[g V_{e}, g V_{s}\right]$ and $\left[g V_{e}, g V_{t}\right]$ emanating from $V=g V_{e}$. We shall denote this edge by $g E$ and the its endpoints by $g S$ and $g T$ respectively. Clearly we have $g S=h S \Longleftrightarrow g^{-1} h \in\langle s\rangle$ and $g T=h T \Longleftrightarrow g^{-1} h \in\langle t\rangle$, while $g S \neq h T$, for all $g, h \in G$. (The vertices of $\operatorname{Lk}(V, \mathbb{D})$ may in fact be thought of as cosets in $G$ of subgroups $S=\langle s\rangle$ and $T=\langle t\rangle$ which lie in a common coset of $G(e))$.

Now suppose that $A$ is a solid chunk of $\Delta$ such that $L_{A}(V, \Theta)$ is non-empty. Equivalently, $A$ contains the vertex $V_{e}$. Then each oriented solid chunk which contributes to $L_{A}(V, \Theta)$ is either of the form $g \mathbf{X}_{A}=\left(g A, g V_{e}, g F_{s}\right)$ or of the form $-g \mathbf{X}_{A}=\left(g A, g V_{e}, g F_{t}\right)$ for some $g \in G$ such that $g V_{e}=V$. It now follows, from the definition of $L(V, \Theta)$ and the above discussion, that mapping the edge $\left(g \mathbf{X}_{A},-g \mathbf{X}_{A}\right)$ to the edge $g E=(g S, g T)$, for each $g$, defines a graph isomorphism.

Finally we note that the graph $L k(V, \mathbb{D})$ is connected (essentially because the group $G(e)$ is generated by $s$ and $t$ ) and that it is clear from the definitions that the different subgraphs $L_{A}(V, \Theta)$ lie in different connected components of $L(V, \Theta)$.

Remark Note that the isomorphism of Proposition 38(ii) is natural in the sense that it is equivariant with respect to the obvious $\operatorname{Stab}(V)$ action on each graph.

Notation (Generic rank 2 vertex link) We shall adopt the notation suggested in the above proof in order to describe the link $L k(V, \mathbb{D})$ of a generic rank 2 vertex $V$ of the Deligne complex $\mathbb{D}$. For simplicity we shall suppose that $V=V_{e}$ where $e=\{s, t\} \in E(\Delta)$ and we shall write $m=m_{e}$. Recall that the stabilizer, $\operatorname{Stab}\left(V_{e}\right)$, of this vertex under the action of $G$ on $\mathbb{D}$ is the group

$$
G(e)=\langle s, t \mid \operatorname{prod}(s, t ; m)=\operatorname{prod}(t, s ; m)\rangle,
$$


where $\operatorname{prod}(s, t ; m)$ denotes the word $s t s \ldots$ of length $m$. Let $S=\langle s\rangle$ and $T=\langle t\rangle$. The vertices of the graph $\operatorname{Lk}\left(V_{e}, \mathbb{D}\right)$ shall be represented by the cosets of the subgroups $S$ and $T$ in $G(e)$ and, for each $g \in G(e)$, there is a single edge with vertices $g S$ and $g T$, written $g E=\{g S, g T\}$. (The symbol $E$ may be thought of as representing the trivial subgroup $E=\{1\})$. The action of $G(e)$ on $\operatorname{Lk}\left(V_{e}, \mathbb{D}\right)$ (coming from the action of $G$ on $\mathbb{D}$ ) is defined in the obvious way, be left multiplication of cosets. Thus $\operatorname{Stab}_{G(e)}(g S)=g\langle s\rangle g^{-1}$, $\operatorname{Stab}_{G(e)}(g T)=g\langle t\rangle g^{-1}$ and $\operatorname{Stab}_{G(e)}(g E)=1$.

We view $G(e)$ as the quotient of the free product $\langle s\rangle \star\langle t\rangle$ by the single relation shown in the above presentation, and we make the following observation. Locally geodesic circuits in $\operatorname{Lk}\left(V_{e}, \mathbb{D}\right)$ which pass through the fundamental edge $E$ correspond bijectively to cyclically reduced expressions over $\langle s\rangle \star\langle t\rangle$ for the identity in $G(e)$, ie, expressions $w=a_{1} a_{2} \ldots a_{n}$ where the $a_{i}$ belong alternately to $\langle s\rangle \backslash\{1\}$ and $\langle t\rangle \backslash\{1\} \quad\left(a_{1}\right.$ and $a_{n}$ belonging to distinct subgroups). To be precise, a cyclically reduced expression for the identity in $G(e)$ which is written $w=a_{1} a_{2} \ldots a_{n}$ corresponds to the circuit $W=\left(E, a_{1} E, a_{1} a_{2} E, \ldots, a_{1} . . a_{n-1} E, w E=E\right)$ of the same length in $\operatorname{Lk}\left(V_{e}, \mathbb{D}\right)$. Note, also, that any circuit in $\operatorname{Lk}\left(V_{e}, \mathbb{D}\right)$ may be translated by a graph automorphism (action by an element of $G(e))$ to a circuit passing through $E$.

Lemma 39 Suppose that $\Delta$ is the defining graph for a 2-dimensional Artin group. Let $e=\{s, t\}$ be an edge of $\Delta$ with label $m=m_{e} \geq 3$, and let $L=L k\left(V_{e}, \mathbb{D}\right)$ denote the link in the Deligne complex of the rank 2 vertex $V_{e}$ fixed by $G(e)$.

Let $w$ denote a nonempty cyclically reduced expression over $\langle s\rangle *\langle t\rangle$ which represents the identity in $G(e)$, and write len $(w)$ for the (syllable) length of $w$. Then $\operatorname{len}(w) \geq 2 m$ and if len $(w)=2 m$ then, up to a cyclic permutation and inversion $\left(w \leftrightarrow w^{-1}\right)$, the word $w$ is one of the following balanced expressions, for some $n \in \mathbb{Z} \backslash\{0\}$,

$$
\begin{aligned}
& s^{n} t \ldots s t\left(t s \ldots t s^{n}\right)^{-1} \quad \text { or } \quad t^{n} s \ldots t s\left(s t \ldots s t^{n}\right)^{-1} \quad \text { if } m \text { even, and } \\
& s^{n} t \ldots t s\left(t s \ldots s t^{n}\right)^{-1} \quad \text { or } \quad t^{n} s \ldots s t\left(s t \ldots t s^{n}\right)^{-1} \quad \text { if } m \text { odd. }
\end{aligned}
$$

Equivalenty, every simple circuit in $L$ has edge length at least $2 m$ and if it has edge length precisely $2 m$ then it is a translate (by some element of $G(e)$ acting on $L$ ) of one of the circuits through $E$ corresponding to the above expressions.

Proof Recall that $G(e)$ acts by isometries on a regular $m$-valent tree $T$ in such a way that the generators $s$ and $t$ are each hyperbolic on $T$, the stabilizer 
of the midpoint of any edge is conjugate to $\langle x\rangle$ where $x=\operatorname{prod}(s, t ; m)$, and the kernel of the action is the centre of $G(e)$, generated by the element $x$ if $m$ is even and $x^{2}$ if $m$ is odd. The tree may be embedded in the plane $\mathbb{R}^{2}$ (and the action extended non-isometrically) in such a way that the axis for each generator $s$ and $t$ (and each of their conjugates) bounds a connected component of $\mathbb{R}^{2} \backslash T$. Moreover, the action is such that the axes for $s$ and $t$ in $T$ intersect along a single edge $A$, but are oriented in opposite directions along this edge.

We let $M$ denote the graph dual to $T$ in the plane. Observe that there is a natural $G(e)$-equivariant map $p: L \rightarrow M$ which sends the edge $E$ to the edge of $M$ dual to $A$, and vertices $S$ and $T$ to the vertices of $M$ lying in the regions bounded by the axes for $s$ and $t$ respectively. This map $p$ is in fact a covering projection. We also observe that any simple closed path $\rho$ in $M$ which starts at a vertex and runs exactly once around the boundary of a single region of $\mathbb{R}^{2} \backslash M$ (thus a path of length $m$ surrounding a single vertex of the tree $T$ ) always lifts to a path in $L$ of the form $g(E, s E, s t E, s t s E, . ., \alpha E)$ for $g \in G$ and $\alpha=\operatorname{prod}(s, t ; m-1)$, or of similar form using the word $\beta=\operatorname{prod}(t, s ; m-1)$ or one of $\alpha^{-1}$ or $\beta^{-1}$ in place of $\alpha$. Also, by choice of orientation of $\mathbb{R}^{2}$, we may suppose that the lift of $\rho$ is associated with a positive word $(\alpha$ or $\beta$ ) if and only if $\rho$ runs in a clockwise direction.

More generally, any simple circuit $\rho$ in $M$ encloses a region containing a finite number of vertices of $T$, and if the simple circuit $\rho$ surrounds exactly $N$ vertices of $T$ then it has length $(m-2) N+2 \geq m$. Moreover, the circuit $\rho$ lifts to a path in $L$ associated with a strictly positive or strictly negative word in the generators $s, t$ depending on whether it is oriented in the clockwise or anticlockwise direction.

Now consider a simple circuit $W$ in the graph $L$, corresponding to a reduced expression $w$ over $\langle s\rangle \star\langle t\rangle$ for the identity in $G(e)$. This projects to a locally geodesic (but not necessarily simple) circuit $\bar{W}$ in $M$. One may easily find a subpath of $\bar{W}$ which describes a simple circuit in $M$. Thus we may decompose $\bar{W}$ into the concatenation of paths $\rho . \rho^{\prime}$ where $\rho$ is a simple circuit. In particular, the length of $\bar{W}$ is at least $m$. Also, $\rho$ lifts to a path in $L$ associated to a word $u$ in $s, t$ which is strictly positive or negative. Such a word cannot represent the identity in $G(e)$, so is not equal to $w$. It follows that $\rho^{\prime}$ is a nontrivial circuit in $M$. Repeating the above argument we have $l\left(\rho^{\prime}\right) \geq m$, and so $l(\bar{W})=l(\rho)+l\left(\rho^{\prime}\right) \geq 2 m$. Moreover, $l(\bar{W})=2 m$ only if both $\rho$ and $\rho^{\prime}$ are simple circuits, each surrounding a single vertex of $T$. Finally, in this case, since $w=1$ in $G(e)$ one of $\rho, \rho^{\prime}$ is oriented clockwise, the other anti-clockwise, 
and $w$ is necessarily given by one of the words listed in the statement of the Lemma.

Proposition 40 Suppose that $\Delta$ is the defining graph for a 2-dimensional Artin group. Let $e=\{s, t\}$ be an edge of $\Delta$ with label $m=m_{e} \geq 3$, and let $L=L k\left(V_{e}, \mathbb{D}\right)$ denote the link in the Deligne complex of the rank 2 vertex $V_{e}$. If $\tau$ is a graph automorphism of $L$ which fixes the fundamental edge $E=\{S, T\}$ (ie, $\tau(S)=S$ and $\tau(T)=T$ ) then, either $\tau$ is the identity on $L$, or it is induced by the group inversion such that $s \mapsto s^{-1}$, and $t \mapsto t^{-1}$.

Proof Consider the circuits of minimal length $2 m$ in $L$ which pass through the fundamental edge $E$. We observe that the edge pair $(E, s E)$ appears in infinitely many minimal length circuits, while $\left(E, s^{k} E\right)$, with $|k|>1$, appears in at most one or two minimal length circuits (depending on whether $m$ is odd or even). This implies that the natural total order on the set $\left\{s^{n} E: n \in \mathbb{Z}\right\}$ (coming from the natural ordering of the integers) is determined up to a reversal of order by graph theoretic information. It follows that, by composing $\tau$ with an inversion automorphism if necessary, we may suppose that $\tau$ is the identity on the neighbourhood of $S$ (ie, the union of edges $s^{k} E$ for $k \in \mathbb{Z}$ ). Also, $\tau(t E)=t E$ or $t^{-1} E$. Note also that, since the canonical cyclic ordering on any vertex of $L$ is respected (up to reversal) by any graph automorphism, the family of minimal length circuits associated to words of total word length $2 \mathrm{~m}$ ( $n= \pm 1$ in Lemma 39) is respected by $\tau$. These are the circuits associated to the following cyclic words and their inverses

$$
\begin{array}{ll}
s t \ldots s t(t s \ldots t s)^{-1} & \text { if } m \text { even, and } \\
s t \ldots t s(t s \ldots s t)^{-1} & \text { if } m \text { odd. }
\end{array}
$$

However, we observe that, in each of these cyclic words, the word $s t^{-1}$ (or its inverse) appears exactly once as a subword, while the word st (or its inverse) appears a total of $m-1$ times. Since $m \geq 3$, the paths $(s E, E, t E)$ and $\left(s E, E, t^{-1} E\right)$ are thus differentiated by the number of minimal length circuits of this type which contain them. Therefore $\tau(t E)=t E$ and in fact $\tau$ must fix the whole neighbourhood of $T$. Since a similar argument may be applied at each edge of $L$, and the graph is connected, it now follows that $\tau$ is the identity on the whole graph.

Propositions 38 and 40 together give the "rigidity in the neighbourhood of a vertex" property that will be needed in the following Section to complete the proof of Theorem 3 . 


\section{Abstract commensurators - Theorem 3}

Throughout this section we suppose that $\Delta, \Delta^{\prime}$ denote CLTTF defining graphs. For simplicity we shall write $G=G(\Delta), G^{\prime}=G\left(\Delta^{\prime}\right)$, and $\Theta, \Theta^{\prime}$ for the associated fixed set graphs respectively.

Proposition 41 If the defining graph $\Delta$ is not a tree (ie $\Delta$ contains at least one simple circuit) then any graph isomorphism $\Theta \rightarrow \Theta^{\prime}$ is a $\mathcal{V} \mathcal{F}$-isomorphism.

Proof Let $\Phi: \Theta \rightarrow \Theta^{\prime}$ be a graph isomorphism. Recall that $\Theta$ and $\Theta^{\prime}$ are connected bi-partite graphs. If $\Phi$ is not a $\mathcal{V} \mathcal{F}$-isomorphism then we may suppose that $\Phi(\mathcal{F})=\mathcal{V}^{\prime}$ and $\Phi(\mathcal{V})=\mathcal{F}^{\prime}$. Since $\Delta$ is not a tree, we may choose some $V \in \mathcal{V}$ which lies in a solid chunk of $\Theta$ (so that $L(V, \Theta) \neq \emptyset$ ). Note that $L(V, \Theta)$ contains many simple closed circuits (cf. Proposition 38 and Lemma 39). Let $X_{1}, X_{2}, \ldots, X_{n}$ denote a sequence of solid chunks in $\Theta$ which represents a simple closed edge path in $L(V, \Theta)$ (each $X_{i}$ contains $V$ ). The image $\Phi\left(X_{i}\right), i=1, . ., n$, of this sequence is a sequence of solid chunks of $\Theta^{\prime}$ all of which contain the vertex $\Phi(V) \in \mathcal{F}^{\prime}$. By an application of Lemma 24(iii), the conditions controlling adjacency of edges in $L(V, \Theta)$ (see Section 10) translate under $\Phi$ to the following condition. For each $i=1, . ., n$, there exists $e_{i} \in E\left(\Delta^{\prime}\right)$ such that

$$
\beta\left(X_{i}\right)^{-1} \beta\left(X_{i+1}\right)=x_{e_{i}}^{m_{i}} \quad \text { for some nonzero } m_{i} \in \mathbb{Z} .
$$

Moreover, we must have $e_{i} \neq e_{i+1}$, for all $i$. (Here indices are taken $\bmod n$ ). But then we have that

$$
x_{e_{1}}^{m_{1}} x_{e_{2}}^{m_{2}} \ldots x_{e_{n}}^{m_{n}}=1,
$$

which contradicts the fact that the elements $\left\{x_{e}: e \in E\left(\Delta^{\prime}\right)\right\}$ freely generate a free group, by Lemma 25.

\subsection{Proof of Theorem 3}

We recall that, in the statement of the Theorem, $\Delta$ denotes a CLTTF defining graph with no separating edge or vertex.

(i) Suppose $G(\Delta)$ is abstractly commensurable to $G\left(\Delta^{\prime}\right)$ for some CLTTF defining graph $\Delta^{\prime}$. We wish to show that $\Delta$ and $\Delta^{\prime}$ are label isomorphic. 
The condition that $\Delta$ has no separating edge or vertex simply means that $\widehat{\Delta}$ is itself a solid chunk of $\Theta$ (the unique fundamental chunk in this case). In particular, $\Delta$ is not a tree and Proposition 41 applies. Let $\varphi \in \operatorname{Comm}\left(G(\Delta), G\left(\Delta^{\prime}\right)\right)$. By Proposition 41 and Proposition [13, $\varphi$ induces a $\mathcal{V} \mathcal{F}$-isomorphism $\Phi: \Theta \rightarrow$ $\Theta^{\prime}$ which, by Proposition 23. maps solid chunks of $\Theta$ to solid chunks of $\Theta^{\prime}$. But then, up to modification of $\varphi$ by an inner automorphism, we may suppose that $A:=\Phi(\widehat{\Delta})$ is a solid chunk of $\widehat{\Delta}^{\prime}$. Thus $\Delta$ is isomorphic to a subgraph of $\Delta^{\prime}$. Moreover, the isomorphism respects labels because the label $m_{e}$ is determined by the structure of the link graph $L\left(V_{e}, \Theta\right)$. Namely, the shortest simple closed path in $L\left(V_{e}, \Theta\right)$ has length $2 m_{e}$ (cf. Lemma 39] and Proposition 38).

Recall that the Deligne complex $\mathbb{D}$ (of type $\Delta$ ) may be described as the universal cover of a complex of groups structure over the fundamental region $K$. We write $\mathbb{D}^{\prime}$ and $K^{\prime}$ for the Deligne complex of type $\Delta^{\prime}$ and its fundamental region. There is a naturally defined subcomplex $K_{A} \subset K^{\prime}$ associated to the fundamental chunk $A$ (which is spanned by those vertices corresponding to standard parabolic subgroups lying in $G(A)$ ), and we define the following subcomplex of $\mathbb{D}^{\prime}$ :

$$
\mathbb{D}_{A}=\bigcup_{g \in G(A)} g K_{A}
$$

Clearly, $\mathbb{D}_{A}$ is an isometric copy of the Deligne complex associated to $G(A)$ sitting inside $\mathbb{D}^{\prime}$. We claim that the map $\Phi: \Theta \rightarrow \Theta^{\prime}$ induces an isometry $\mathbb{D} \rightarrow \mathbb{D}_{A}$.

The fact that $\Phi$ maps chunks of $\Theta$ to solid chunks of $\Theta^{\prime}$ means that there is a naturally induced family of isomorphisms

$$
g K \rightarrow \phi(g) K_{B(g)} \quad \text { for each } g \in G(\Delta) .
$$

where $\phi: G(\Delta) \rightarrow G\left(\Delta^{\prime}\right)$ and $B: G(\Delta) \rightarrow C h k\left(\Delta^{\prime}\right)$ are simply functions. By the discussion in the opening paragraph, We have that $\phi(1)=1, B(1)=A$, and the map $K \rightarrow K_{A}$ is induced by a label isomorphism between the graphs $\Delta$ and $A$.

Let $e \in E(\Delta)$, and $e^{\prime}$ its image in $A$. Then, by Proposition 38, the $\mathcal{V F}-$ isomorphism $\Phi$ must induce an isomorphism $L\left(V_{e}, \Theta\right) \cong L\left(V_{e^{\prime}}, \Theta^{\prime}\right)$, and there is a naturally induced isomorphism $L k\left(V_{e}, \mathbb{D}\right) \cong L k\left(V_{e^{\prime}}, \mathbb{D}^{\prime}\right)$. In particular, the function $\phi$ restricts to an isomorphism $G(e) \rightarrow G\left(e^{\prime}\right)<G(A)$ and $B(g)=A$ for all $g \in G(e)$. This naturally induces a well-defined isometric embedding of the neighbourhood of a rank 2 vertex of $\mathbb{D}$ into $\mathbb{D}_{A}$. Applying the same argument at every rank 2 vertex of $\mathbb{D}$ and using the fact that $\mathbb{D}$ is connected we obtain a map $\Phi_{\mathbb{D}}: \mathbb{D} \rightarrow \mathbb{D}_{A}$ which is locally isometric, so globally isometric 
since $\mathbb{D}$ is $\operatorname{CAT}(0)$. The isomorphism $\Phi_{\mathbb{D}}$ is natural in the sense that if $H<G$ is the domain of $\varphi$, then $\operatorname{Stab}_{\varphi(H)}\left(\Phi_{\mathbb{D}}(p)\right)=\varphi\left(\operatorname{Stab}_{H}(p)\right)$ for all vertices $p \in \mathbb{D}$.

It follows from the above argument that the image of the abstract commensurator $\varphi$ is a finite index subgroup of $G(A)$. However, since $G(A)$ is infinite index in $G\left(\Delta^{\prime}\right)$ unless $A=\Delta^{\prime}$, statement (i) of Theorem 3 follows.

(ii) Suppose now that $\Delta$ satisfies the vertex rigidity condition:

(VR) Any label preserving automorphism of $\Delta$ which fixes the neighbourhood of a vertex is the identity automorphism,

and write $G=G(\Delta)$. We wish to show that $\operatorname{Comm}(G) \cong \operatorname{Aut}(G)$.

As in the proof of part (i) (with $\Delta^{\prime}=\Delta$ ), we may suppose, up to modification of $\varphi$ by an inner automorphism and a graph automorphism of $G$, that $\varphi$ naturally induces an isometry $\Phi_{\mathbb{D}}$ of $\mathbb{D}$ which is the identity on the fundamental region $K$. Moreover, by Lemma 40] $\Phi_{\mathbb{D}}$ induces either the identity or the "inversion" automorphism on the link of each rank 2 vertex in $K$. Fixing $e \in E(\Delta)$, we may suppose, up to modification of $\varphi$ by a global inversion if necessary, that $\Phi_{\mathbb{D}}$ induces the identity on $\operatorname{Lk}\left(V_{e}, \mathbb{D}\right)$, and hence restricts to the identity on a small open neighbourhood of $V_{e}$ in $\mathbb{D}$.

We now use the hypothesis that $\Delta$ satisfies the vertex rigidity condition (VR) to show that $\Phi_{\mathbb{D}}$ is the identity on the whole of $\mathbb{D}$. On the one hand, if $\Phi_{\mathbb{D}}$ is the identity on a small open neighbourhood of any rank 2 vertex $p$ of $\mathbb{D}$ then by (VR) it is the identity on every translate of $K$ adjacent to this vertex. On the other hand, if $p$ and $q$ are rank 2 vertices joined by a path $(p, r, q)$ in $\mathbb{F}$, where $r$ is a rank 1 vertex, then we observe that every translate of $K$ adjacent to $r$ is also adjacent to both $p$ and $q$. If $\Phi_{\mathbb{D}}$ is the identity on every translate of $K$ adjacent to $p$ then, since at least two, in fact infinitely many, of these translates are also adjacent to $r$ and $q$, it follows, by Lemma 40, that $\Phi_{\mathbb{D}}$ induces the identity on the link of $q$. This argument, together with the statement involving the (VR) hypothesis, shows that if $\Phi_{\mathbb{D}}$ is the identity on the neighbourhood of $p$ then it is the identity on every translate of $K$ which is adjacent to a rank 2 vertex $q$ within a ball of radius 2 about $p$ in the Deligne complex. Since, from the previous paragraph, we may suppose that $\Phi_{\mathbb{D}}$ is the identity on a neighbourhood of the vertex $V_{e}$, by applying this argument inductively and appealing to the connectedness of $\mathbb{D}$, we show that the map $\Phi_{\mathbb{D}}$ must be the identity on the neighbourhood of every rank 2 vertex in $\mathbb{D}$, and therefore equal to the identity everywhere. 
Finally, if $\Phi_{\mathbb{D}}$ is the identity then $\varphi$ must also be the identity (since for each $\left.h \in H, \Phi_{\mathbb{D}}(h K)=\varphi(h) K\right)$. This completes the proof of statement (ii) of Theorem 3 .

\section{Examples of nontrivial abstract commensurators}

We conclude by giving some examples of abstract commensurators which illustrate the situations one might need to consider in order to extend Theorem 3 , We begin with the necessity of the (VR) hypothesis in part (ii) of the Theorem.

Example Let $\Delta$ be the CLTTF defining graph shown in Figure 3(i). Note that $\Delta$ has no separating edge or vertex, but does not satisfy the vertex rigidity condition (VR). The standard generators of $G(\Delta)$ are labelled $u, v, x, y, z$ as shown in the Figure. Let $X$ denote the presentation $2-$ complex of the standard presentation of $G=G(\Delta)$ given in the introduction. Thus $X$ has a single vertex, an oriented labelled 1-cell for each of the generators, and a 2-cell corresponding to each relator in the presentation, and $\pi_{1}(X)=G$.

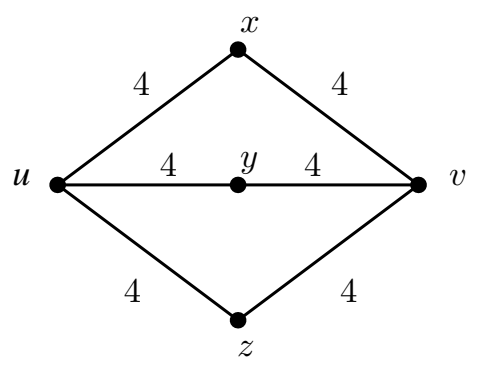

(i)

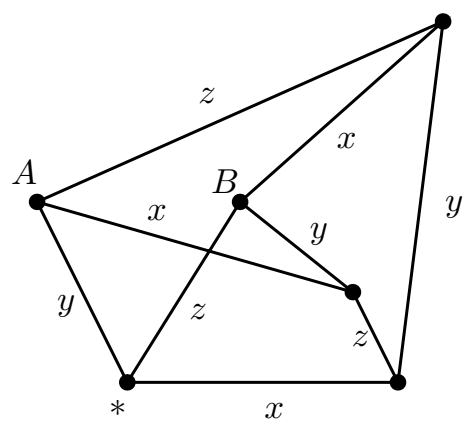

(ii)

Figure 3: (i) A non- "vertex rigid" defining graph $\Delta$ and (ii) a recipe $L$ for an index 6 subgroup of $G=G(\Delta)$ which admits an automorphism not induced from an element of $\operatorname{Aut}(G)$

The labelled graph $L$ shown in Figure 3(ii) is a recipe for building a finite index cover $\widetilde{X}$ of $X$, and so represents a finite index subgroup of $G$, as follows. Let the vertices of $\widetilde{X}$ be in bijection with the vertices of $L$. For each edge $(P, Q)$ in $L$ labelled with a generator $w$ of $G$ there are two oriented 1-cells between $P$ and $Q$ in $\widetilde{X}$, each labelled $w$, one oriented from $P$ to $Q$, and the other in 
the opposite sense. At each vertex $P, \tilde{X}$ has an oriented 1-cell (a loop from $P$ back to $P$ ) labelled $u$, and another labelled $v$. This defines the 1-skeleton of $\widetilde{X}$. Note that the labelling and orientation define a 6 -fold covering map $\widetilde{X}^{(1)} \rightarrow X^{(1)}$. We now define the 2 -cells of $\widetilde{X}$ in the unique way that will enable us to extend this covering map to a 6 -fold covering map $\widetilde{X} \rightarrow X$. Note that there is no obstruction to doing this because every edge label in $\Delta$ is even. Finally, choose a basepoint $*$ for $\widetilde{X}$ as indicated in Figure 3(ii).

Let $H=\pi_{1}(\widetilde{X}, *)$ denote the index 6 subgroup of $G$ associated with this covering map and observe that any automorphism of the underlying graph of $L$ induces an automorphism of the group $H$. Let $\varphi: H \rightarrow H$ denote the automorphism induced by exchanging the vertices labelled $A$ and $B$ in Figure 3(ii), and leaving all other vertices of $L$ fixed.

We remark that $\varphi$ is not induced by any automorphism of $G$. To see this, we note that $x^{2}, y^{2}, z^{2}, u$ and $v$ are all elements of $H$ and $\varphi$ exchanges $y^{2}$ and $z^{2}$ while fixing $x^{2}, u$, and $v$. Therefore the only candidate for an element of $\operatorname{Aut}(G)$ which induces $\varphi$ would be the graph automorphism $\tau$ which exchanges generators $y$ and $z$, leaving all other generators fixed. However $\varphi$ also fixes the element $x z^{2} x^{-1}$ while $\tau\left(x z^{2} x^{-1}\right)=x y^{2} x^{-1}$. Thus $\varphi$ and $\tau$ are inequivalent as elements of $\operatorname{Comm}(G)$.

We state the next example in the form of a lemma:

Lemma 42 Let $\Delta, \Delta^{\prime}$ denote Artin defining graphs. Suppose that $e=\{s, t\} \in$ $E(\Delta)$ is a cut edge of $\Delta$, equivalently, $e$ is itself a (non-solid) chunk of $\Delta$. Suppose moreover that $\Delta$ and $\Delta^{\prime}$ differ only in the label on the edge $e$, but that this label is at least 3 in each case. Then the Artin groups $G(\Delta)$ and $G\left(\Delta^{\prime}\right)$ are abstractly commensurable.

Proof If $A \subset \Delta$ is any full subgraph, and $n \geq 1$, then we write $H(A ; n)$ for the index $n$ subgroup of $G(A)$ which is the kernel of the mod $n$ length function (the group of elements $x$ such that $\ell(x) \equiv 0 \bmod n$ ).

Let $e=\{s, t\}$ be an edge with label $m_{e} \geq 3$. Let $k=1 \mathrm{~cm}\left(m_{e}, 2\right)$ and let $n$ be any positive multiple of $k$. Then, since the order of every torsion element of $G(e) / Z$ divides $k$, it follows that $H(e ; n) \cong F \times \mathbb{Z}$ where $F$ is a finitely generated nonabelian free group (nonabelian since $m_{e} \geq 3$ ). Moreover, up to isomorphism of $F \times \mathbb{Z}$, we may suppose that the subgroups $H(s ; n)=H(e ; n) \cap\langle s\rangle=\left\langle s^{n}\right\rangle$ and $H(t ; n)=H(e ; n) \cap\langle t\rangle=\left\langle t^{n}\right\rangle$ are free factors of the subgroup $F$. That is $H(e ; n)=\left(F^{\prime} \star\left\langle s^{n}\right\rangle \star\left\langle t^{n}\right\rangle\right) \times \mathbb{Z}$. Since all finite rank free groups are abstractly 
commensurable we may suppose, up to an abstract commensurator which fixes the subgroups $\left\langle s^{n}\right\rangle$ and $\left\langle t^{n}\right\rangle$, that the rank of $F^{\prime}$ is any given integer. It follows that, if $e_{i}=\left\{s_{i}, t_{i}\right\}$ are edges, for $i=1,2$, with labels $m_{i} \geq 3$ respectively, then $G\left(e_{1}\right)$ and $G\left(e_{2}\right)$ are abstractly commensurable by a commensurator which maps $s_{1}^{n} \mapsto s_{2}^{n}$ and $t_{1}^{n} \mapsto t_{2}^{n}$, for sufficiently large $n$ (we may take $n=k_{1} k_{2}$ where $\left.k_{i}=\operatorname{lcm}\left(m_{i}, 2\right)\right)$.

Now suppose that $e=\{s, t\}$ is a cut edge of the defining graph $\Delta$, and write $\Delta=\Delta_{1} \cup_{s} e \cup_{t} \Delta_{2}$. Then $G(\Delta)$ is an amalgmated product

$$
G(\Delta)=G\left(\Delta_{1}\right){ }_{\langle s\rangle} G(e) \star\langle t\rangle G\left(\Delta_{2}\right) .
$$

We consider two choices of the edge label $m_{e}$, writing $\Delta, \Delta^{\prime}$ for the two defining graphs thus obtained, and $k, k^{\prime}$ for the corresponding values of $1 \mathrm{~cm}\left(m_{e}, 2\right)$. Let $n=k k^{\prime}$. Then the subgroup $H(\Delta ; n)$ is written as an amalgamated product as follows

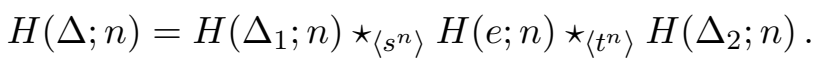

It follows from remarks in the previous paragraph that $H(\Delta ; n)$ and $H\left(\Delta^{\prime} ; n\right)$ are abstractly commensurable. Thus $G(\Delta)$ and $G\left(\Delta^{\prime}\right)$ are abstractly commensurable.

It would be interesting to give a classification of all CLTTF Artin groups up to abstract commensurability. Theorem 3 gives a partial result in this direction. The above Lemma shows that, in order to give a complete treatment of the question, it suffices to consider only those CLTTF defining graphs where every cut edge (equivalently, every edge that does not lie in a circuit) is labelled 3. Moreover, by applying twist isomorphisms we may further restrict our attention to the case where every such edge contains a terminal vertex. The following example suggests that even amongst these defining graphs there may be many non-obvious commensurations.

Lemma 43 Let $\Delta$ denote an arbitrary defining graph, and let $s \in V(\Delta)$. Let $\left(\Delta_{i}, s_{i}\right)$ denote a label isomorphic copy of $(\Delta, s)$, for each $i \in \mathbb{N}$, and let $E$ denote the graph consisting of a single edge $E=\left\{s_{0}, t\right\}$ with label $m_{E}=3$. For $n \in \mathbb{N}$ we write $\Delta^{(n)}$ for the union of the labelled graphs $E, \Delta_{1}, \ldots, \Delta_{n}$ with the vertices $s_{0}, s_{1}, . ., s_{n}$ identified to a single vertex $s$.

Then the Artin groups $G\left(\Delta^{(n)}\right)$ and $G\left(\Delta^{(m)}\right)$ are abstractly commensurable for all $m, n \geq 1$.

Proof We note that, for a sufficiently large $k \in \mathbb{N}, G\left(\Delta^{(1)}\right)$ is abstractly commensurable to $G_{1}:=H(\Delta ; k) \star_{C}((C \star F) \times \mathbb{Z})$ where $C$ denotes the cyclic 
subgroup of $G\left(\Delta^{(1)}\right)$ generated by $s^{k}$, and $F$ denotes a free group of finite rank at least 2. More generally $G\left(\Delta^{(n)}\right)$ is abstractly commensurable to the amalgamated product

$$
G_{n}:=H\left(\Delta_{1} ; k\right) \star_{C} \cdots \star_{C} H\left(\Delta_{n} ; k\right) \star_{C}((C \star F) \times \mathbb{Z}) .
$$

We now observe that the group $G_{n}$ is isomorphic to an index $n$ subgroup of $G_{1}$. Let $\lambda: G_{1} \rightarrow \mathbb{Z}$ denote the surjective homomorphism whose kernel contains the free factor $H(\Delta ; k)$ as well as the subgroup $C \star F$ of the remaining factor. Then $G_{n}$ is isomorphic to the kernel of the quotient map $G_{1} \rightarrow \mathbb{Z} / n \mathbb{Z}$ which factors through $\lambda$. Thus, each group $G_{n}$ is abstractly commensurable to $G_{1}$, completing the proof.

\section{References}

[1] E Artin, Braids and permutations, Ann. of Math. 48 (1947) 643-649 MathReview

[2] P Bahls, Automorphisms of Coxeter groups, Trans. Amer. Math. Soc. (to appear)

[3] P Bahls, Rigidity of 2-dimensional Coxeter groups, preprint (2004)

[4] N Brady, J McCammond, B Mühlherr, W Neumann, Rigidity of Coxeter groups and Artin groups, Geom. Dedicata 94 (2002) 91-109 MathReview

[5] N Brady, J Crisp, Two dimensional Artin groups with CAT(0) dimension three, Geometriae Dedicata 94 (2002) 185-214 MathReview

[6] M R Bridson, On the semisimplicity of polyhedral isometries, Proc. Amer. Math. Soc. 127 (1999) 2143-2146 MathReview

[7] M R Bridson, A Haefliger, Metric Spaces of Non-Positive Curvature, Grundlehren series 319, Springer-Verlag (1999) MathReview

[8] R Charney, J. Crisp, Automorphism groups of some affine and finite type Artin groups, Math. Res. Letters 12 (2005) 321-333

[9] R Charney, M W Davis, The $K(\pi, 1)$-problem for hyperplane complements associated to infinite reflection groups, J. Amer. Math. Soc. 8 (1995) 597-627 MathReview

[10] A M Cohen, L Paris, On a theorem of Artin, J. Group Theory 6 (2003) 421441 MathReview

[11] C Droms, Isomorphisms of graph groups, Proc. Amer. Math. Soc. 100 (1987) 407-408 MathReview

[12] N D Gilbert, J Howie, V Metaftsis, E Raptis, Tree actions of automorphism groups, J. Group Theory 3 (2000) 213-223 MathReview 
[13] E Godelle, Parabolic subgroups of Artin groups of type FC, Pacific J. Math. 208 (2003) 243-254 MathReview

[14] E Godelle, Artin-Tits groups with CAT(0) Deligne complex, preprint

[15] $\mathbf{H}$ van der Lek, The Homotopy Type of Complex Hyperplane Complements, PhD Thesis, University of Nijmegen (1983)

[16] G Moussong, Hyperbolic Coxeter groups, PhD Thesis, Ohio State University (1988)

[17] B Mühlherr, The isomorphism problem for Coxeter groups, from: "The Coxeter Legacy: Reflections and Projections", Fields Institute Communications (to appear)

[18] B Mühlherr, R Weidmann, Rigidity of skew-angled Coxeter groups, Adv. Geom. 2 (2002) 391-451 MathReview

[19] G Niblo, L Reeves, The geometry of cube complexes and the complexity of their fundamental groups, Topology 37 (1998) 621-633 MathReview

[20] L Paris, Artin groups of spherical type up to isomorphism, J. Algebra 281 (2004) 666-678 MathReview

[21] M Sageev, Ends of group pairs and non-positively curved cube complexes, Proc. London Math. Soc. 71 (1995) 585-617 MathReview 